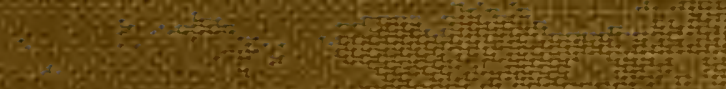

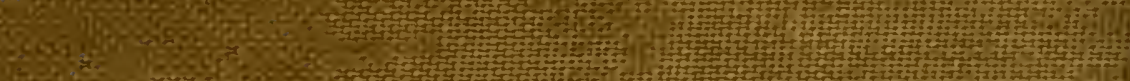

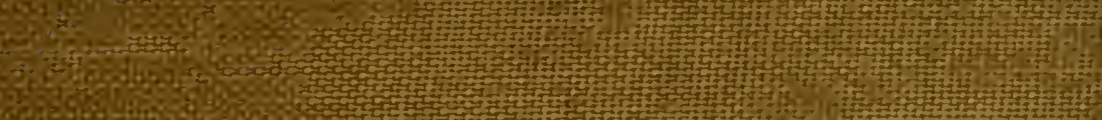

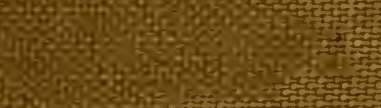

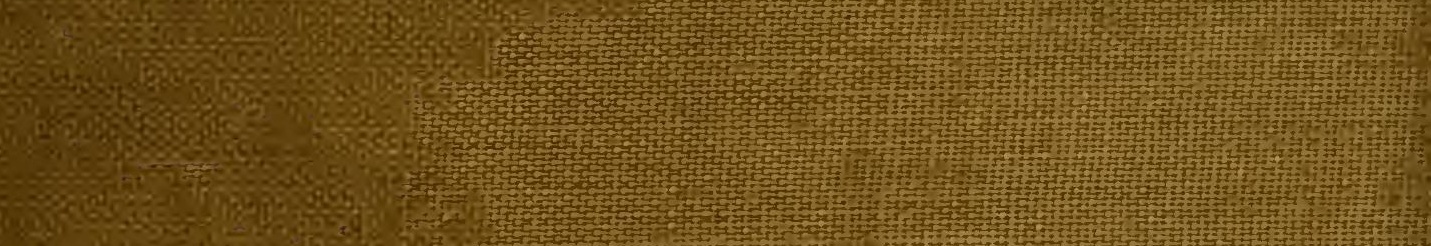

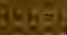
किस 10

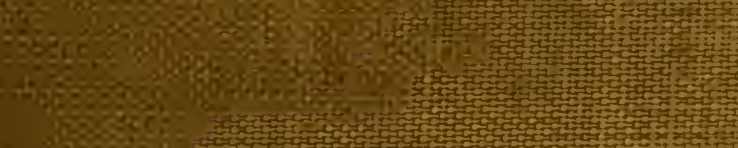

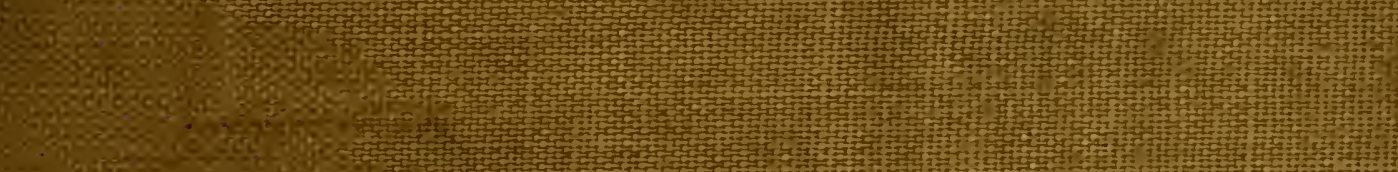
4

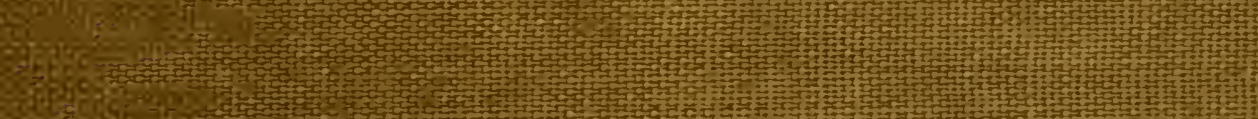

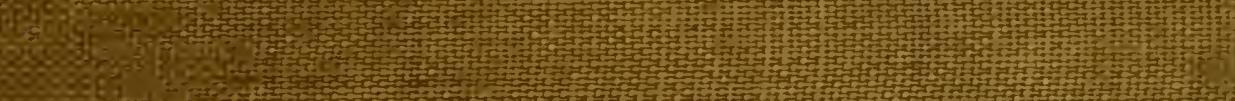

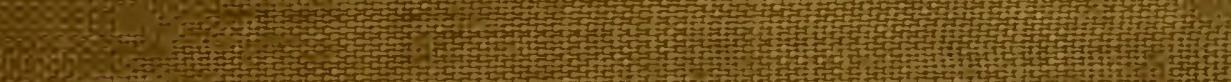

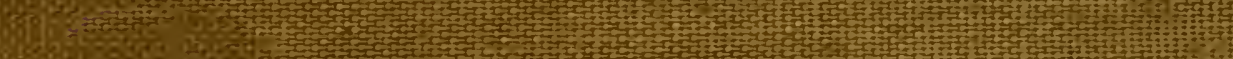
(1) 


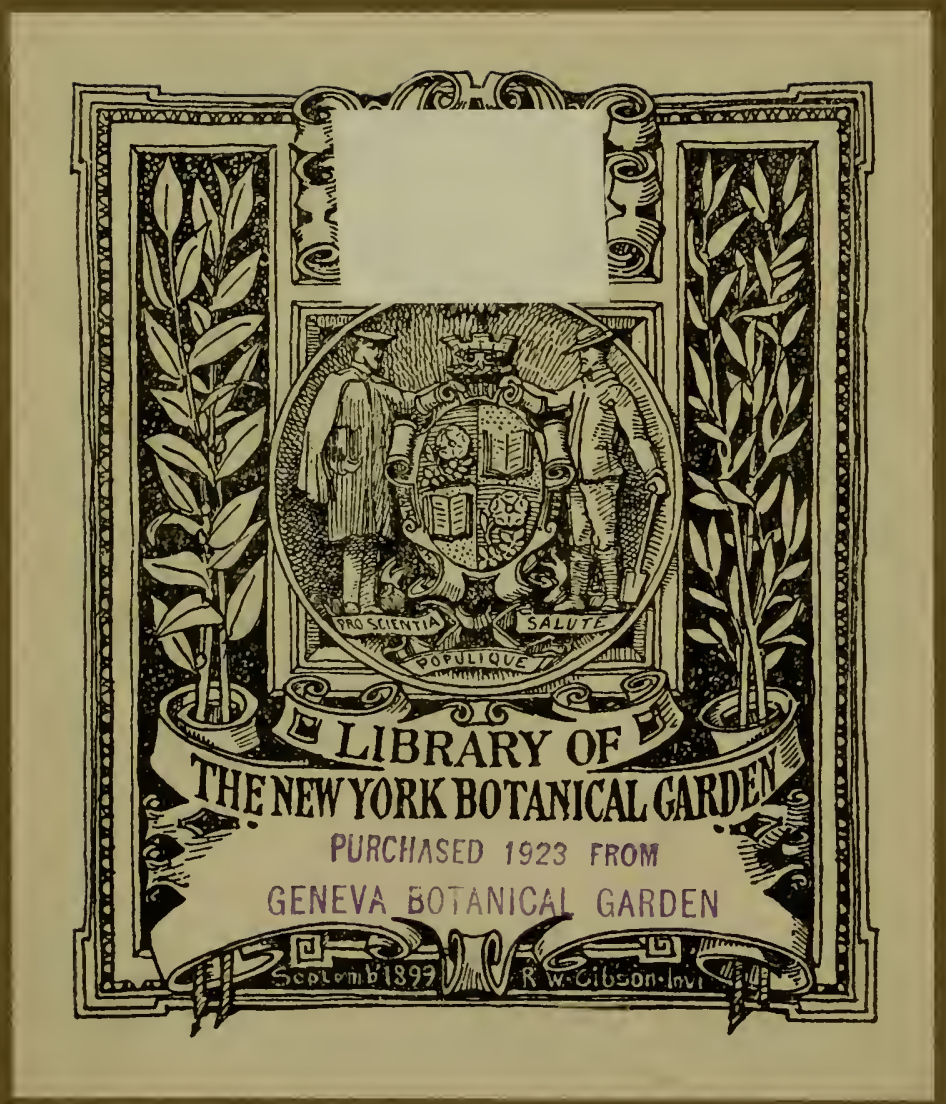




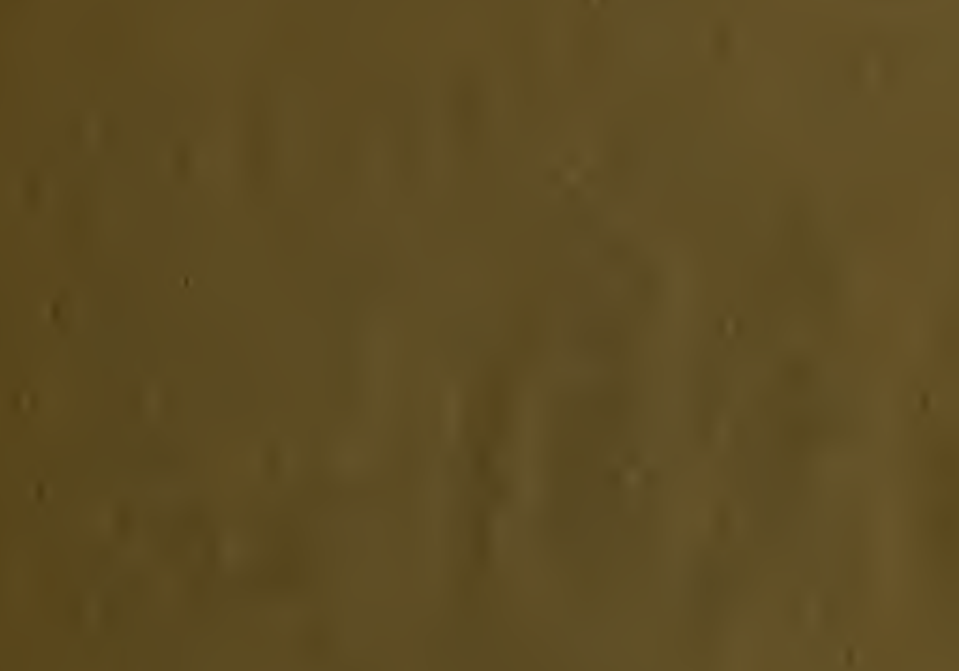

11) 


\title{
RECHERCHES
}

\author{
S UR L A
}

\section{GEOGRLPHIE BOTANIOLE}

D $\mathrm{C}$

\section{LYONNAIS}

\author{
P. $1 R$ \\ ANTOLNE MAGNIN \\ DOCTELR EN MUDECISE \\ DOCTEUR ES-SCIENCES NATURELLES, SECRETAIRE FE SERAL \\ DE LA SOCIÉTE BOTAXIUUE DE L XOX, ETC.
}

BAS-PI.ATEAUX LYONNAIS

COTIËRE MÉRIDIONALE DE LA DOMBES

- IVer DEEI CIRTES enlopíes -

\section{PARIS}

LIBR.IIRIE J.-B. BAILLIERLET FILS

19, rue IIanteteuille, pris du boulevard Saint-Germain

\section{LONDIES}

EALLLLHE, TINDALL AND GOX

:n. Kinn Witliam street

\section{MADRXD}

CAHLOS NAILTX-BAILLTIERE Plaza de Topete, s 
- 
DUPLICATA DE LA BIBLIOTHÊQUE DU CONSHRVATOIRE BOTANIQUE DE GENEVE VENDU EN 1922 


\title{
ERRATA ET GORREGTIONS
}

\author{
P. 12, 1. 26, lisez : ses coteaux. \\ P. 18, 1. 13, - dans les couches plus profondes. \\ P. 23, 1. 12 et 13, - En partant... on rencontre successiveinent. \\ P. 24, ]. 1', _- les variations de la température. \\ P. 25, 1. 15, - Ces modifications. \\ - 1.2', - F́tude particulière... \\ P. 26, 1.5, - Distribution des végétaux. \\ P. 36, 1. 25, - Appellerons. \\ P. $44,1.16$, reportez le membre de phrase: " et au printemps, Polentilla verna, Pulsa- \\ P. 48, dernière ligne, lisez : p. 42. \\ tilla rubra » à la ligne 11, aprés Dianthus prolifer. \\ P. 52, - - - p. 42 . \\ P. 55, - - - p. 49 . \\ P. 58, - - - Senecio. \\ P. 62, 1.8, - et l'Umbilicus. \\ P. 91 , dernière ligne, - p. 37. \\ P. $105,1.29, \quad-\quad$ les plateaux. \\ P. 117, 1. 15, - p. 105. \\ P. 125, l. 20, - de la falaise occidentale et méridionale de la Do:nbes, au licu \\ P. 129, 1. 37, - p. 37. \\ P.138, av.-dern.ligne, - MUCGLXXX, au lieu de : MDGCGLXXX. \\ P. 139 dernière ligne, supprimez note 1 . \\ P. 140, 1. 26, supprimes note 1 . \\ P. 142, 1. 34, lisez p. 82 . \\ P. 143, note, ajoutez: Cf. Contejean (Ann. Sc. natur., loc. cit.) \\ P. 147, 1. 1/, lisez : Hylocomium.
}




\section{REGHERCHES}

SUR LA

\section{GEOGRAPHIE BOTANIQUE v U}

LYONNAIS 


\section{ALTRES PUBLIGATIONS DE L'AUTEUR}

1873. Miscelladiés mycologrques: I. Les Entophytes du Jardin botanique de Lyon. - If. Coleosporiun Cacalize. - III. Variétés du Phragmialium bulbosum. (Extr, des Ann. Soc. bol. de Lyon).

- Excursion botanique à Hauterille (Ain). (Ibid.)

- Observations sur les Urédinées (Associvtion française pour l'avancemont des scicn. ces, session de Lyon).

1874. Sur l'envahissement du Puccinia malvacearum dans le Jura et les environs de Lyon. (Extr, des Ann. Soc, bat. de Lyon.)

- Sur une nouvelle localité du Carcx brevicollis dans le Bugey, à Tenay. (Ibid.)

- Diabète sucré avec tumeur cérébrale (cholestéatome du Pont de Varole. (Lyon mé. dical.)

1575. Revue de botanique pharmaceutique dans le journal La Pharmacie de Lyon.

- Artériectasie ancienine. (Lyon médicul.)

- Aperçu de la végétation des environs de Gap.(Extr. des Anu. Soc. bot. Lyon, reproduit dans Bull. Soc, bot. France, session de Gap.)

- Compte rendu de l'excursion au Col-de-Glaise. (In.8, 12 p., ext. du Bull. Soc. bnt. France, session de Gap.)

- Origine glaciaire de la végétation des marais des Echets et du Lyonnais, in-8, 12 p. (Jbid.)

1876. Compte rendu de la session d'Angers. (Extr. des Ann. Soc. bot. Lyon.)

- Sur les conditions qui favorisent le développement du Porphyridium cruentum.(Ibid.)

- Mousses et Lichens du bassin de l'Ubaye. (Extr. du Bull. Soc. hot. France.)

- Recherches géologiques, botaniques et statistiques sur l"impaludisme dans la Dombes et le miasme paludéen; in-S, $120 \mathrm{p}, 1 \mathrm{pl}$. et 8 tableaux. (Th, couronnée pur la Fac. de médecine de Paris, médaille d'argent.)

- Nouvelle espère d'Orbicula._-Sur le Sphrerohleca Caslagnei des Cucurbitacées. (Bull. Soc. bot. France, session mycologique.)

187\%. Résumé des principaux travaux publiés sur les Planles carnivores; in-S, 19 p. (Extr. du Bull. Soc. d'éludes scientifiques.)

- Étude sur les Lichens des vallées de l'thaye et de l'Ubayette (Basses-Alpes); in- $8,16 \mathrm{p}$. (Extr. des Ann. Soc. bot. Lyon.)

- Végétation du rebord méridional de la Dombes, in-\&, z p. (Ibid.)

1878. Les Lichens utiles. (Extr. du Bull. Soc. d'átudes scientifiques.)

- Notes sur l'hahitat anormal d'un Coprin, sur la tératolugie des Saules, etc. (Bull. Soc. bot. Lyon.)

- Les Bactéries. 1 vol. in-\$, 180 p. Paris, Savy.

EN PRËPARATION

RECHERCIES SLR LA GÉOGR.IPHE BOTHNIOUE DU LIONXIS

2. Fascicule. - Coteaux du Rhône. - Mont-d'Or. - Dombes.

3. Fascicule, - Climatologie. - Variations de la Flore lyonnaise.

FLORE DES LICHENS DE LA RÉGIOY LYONXAISE

I. YOX. - IMPRJMERE PJTRAT, AIXE, RUE GENTL, 4. 


\title{
REGHERCHES
}

SUR LA

\section{GEOGRAPHIE BOTANLLE}

D L

\section{LYONNAIS}

\author{
PA A \\ ANTOINE MAGNIN \\ DOCTEER EN MLDECLXI \\ DOCTELR ĹS-SCLENCES NATURELLES, SECRÉTAIRE G ÉNERAL

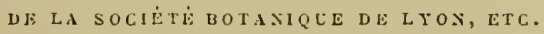

\section{LIBRART \\ NEW YORK \\ BOTANICAL \\ UARUEN}

BAS-PLATEAUX LYONNAIS

COTIÉRE MÉRIDIONALE DE LA DOMBES

- Lree deux cartes coloriées -

\section{PARIS}

LIBRAIRIE J.-B. BAILLIERE ET FILS

19, rue Hautefeuitle, près du buulevard Saint-Germain

\section{LONDEES}

BAILLIERE, TINDALL AND COX

:0, King Wiltiam strect

\section{MADRID}

CARLOS BAILLX-BAILLIERE Plaza de Topete, S 
. M251 


\section{TABLE DES MATIERES}

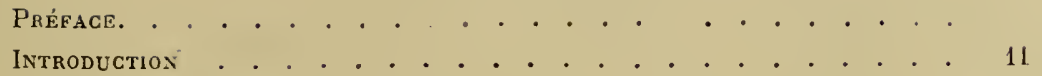

Limites de la région lyonnaise. . . . . . . . . . . 14

Division en régions secondaires : I. Lyonnais granitique; II. Mont-d"Or;

III. Coteaux du Rhòne et de la Saùne et Bas-Dauphiné; IV. Plateau bressan. . . . . . . . . . . . . . . . . .

Comparaison de la végétation de ces régions secondaires au point de vue des : Influence de la composition chimique du sol; Influence de son état physique; Influence de l'exposition; Influence de l'altitude, etc. . . 19

Variations de la Flore: Végétaux adventices. . . . . . . . 2 '

\section{PREMIĖRE PARTIE}

\section{Vallées et Bas-Plateaux du Lyonnais}

Chapitre premier. - Topographie. . . . . . . . . . . 24

Girap. II. - Constitution géologique. . . . . . . . . . . . . 33

Chap. III. - Nature du sol. . . . . . . . . . . . . . . . . 36

Chrp. IV. - Végétation. . . . . . . . . . . . . . . . 42

\$1. Exemples d'herborisations. . . . . . . . . . . . . . 42

$\$$ 2. Énumération des plantes daprès leurs stations. . . . . . . . 60

A. Sous-région granitique. . . . . . . . . . . . 60

B. Sous-région des alluvions glaciaires alpines siliceuses. . . . . 72

§ 3. Ėumération systèmatique des espèces. . . . . . . . . 75

A. Plantes triviales ou communes à toutes les régions. . . . . . $i 5$

B. Plantes moins communes, plus spéciales à la région granitique. is

C. Plantes préfèrant les sols siliceux. . . . . . . . 83

$\$$ 4. Considerations gènérales. . . . . . . . . . . . 88

A. Caractères de la Flore . . . . . . . . . . 88 
10 Influence chimique du sol. . . . . . . . . . . 88

$2 \circ$ Influence physique. . . . . . . . . . . . . . . 89

3 Influence du climat et de l'exposition. . . . . . . . . 89

$1^{\circ}$ Zone méridionale . . . . . . . . . . . . . 90

$2^{\circ}$ Zone septentrionale. . . . . . . . . . . . . . 91

$4^{\circ}$ Flore montagneuse. . . . . . . . . . . . . . 92

B. Comparaison de la régétation du Bas-Lyonnais avec les .

$11^{\circ}$ Alluvions de l'Azergue. . . . . . . . . . . . . 94

20 Cornes vertes. . . . . . . . . . . . . . . . . . . 95

30 Monts du Lyonnais. . . . . . . . . . . . . . 96

40 Plaine du Forez. . . . . . . . . . . . . 98

\section{DEUXIĖME PARTIE}

\section{Coteaux et vallées du Roóne et de la Saóne}

\section{PREMIEUE SECTION：COTIÉRE OCCIDEXTALE ET MERIDIONALE DE LA DOMBES}

Chapttre tremier. - Aperçu topographique. . . . . . . . . . 102

Cinap. II - Géologie et nature du sol. . . . . . . . . . . . . 106

Crap. III. - Végétation, . . . . . . . . . . . . . . . . 110

$\$ 1$ - Énumération des plantes parlerborisation. . . . . . . . . 110

$\$ 2$. Enumération des plantes d'après leurs stations. . . . . . . . . 122

§ 3 . - Ennmération systématique des plantes caractéristiques de la cútière occidentale et méridionale de la Dombes. . . . . . . 125

$\$ 4$ - Caractères le la Flore . . . . . . . . . . . 127

\section{APPENDICE}

Cinpitre premer. - Influence du sol sur la régétation ; - historique. . . Canr. II. - Analogies et contrastes présentées par la végétation des diverses parties de la Flore lyonnaise. . . . . . . . 140

Cinap. III. - Exemples de contrastes en petit, pris dans dautres régions. . . 151

Expligation Des cartes. • . . . . . . . . . . . . . 167 


\section{PRÉFAGE}

En 1869, familiarisé avec la régétation des montagnes calcaires du Jura que j'ètudiais depuis sept ans, soit dans le Bugey, soit dans le Jura proprement dit, je quittai Bellcy pour venir me fixer à Lyon. Je ne connaissais la Flore du Lyonnais que par les livres ou les herbiers; aussi, bien que je me fusse prẻparé à ce changement, dès les premières promenades dans les environs de ma nouvelle résidence, je fus vivement frappé des différences que présentait leur tapis végétal avec celui que j'avais coutume de voir clans mes her borisations antérieures.

D'un côté, c'ètaient des plantes qui m'étaient presque nouvelles, ou que je n'avais jamais vues en telle abondance, comme les Ornithopus, Teesdalia, Nardus, les Scléranthc, le Sarothamne, etc.; d'antre part, c'ètaiert des espèces vul gaires dans le Jura et y habitant assez bas pour qu'on ne puisse pas invoquer l'influence de diffèrences d'altitudes, telles que Rumex scutatus, Cytisus Laburnum, Saponaria ocy- 
moides, Daphne Laureola, etc., qui ne se présentaient plus à mes regards.

Plus tard, j'explorai le Mont-d'Or lyonnais et j'y retrouvai le Buis, le Gytise Aubour, la Goronille Faux-Sèné, la Lauréole des montagnes jurassiques ; bien plus, j'y rencontrai le Lonicera etrusca de la montagne de Parves, et le Montd'Or me rappela le Bugey que je venais de quitter. Et ce ne fut pas seulement ce massif calcaire qui me permit de faire ces rapprochements : je récoltai des plantes jurassiques, le Dianthus Scheuchzeri par exemple, si caractéristique, sur les conglomérats de la Pape, sur les mollasses de Saint.-.Fons; jc voyais même quelques-unes de ces espèces franchir le Rhône et s'établir au sein de la région granitique, dans un terrain spécial, il est vrai, analogue à celui de la Pape, les alluvions glaciaires.

Ces contrastes et ces analogies me frappèrent alors vivement; aussi, depuis cette époque, je me suis constamment préoccupé de ces questions de relation du sol avec la végétation. Si d'autres études incidentes m'ont fait interrompre à diverses l'eprises les recherches que j'accomplissais spécialement dans cette direction, je puis dire que je ne les ai jamais complètement perdues de vue et qu'il ne s'est pas passé d'année où je n'aie recueilli quelques matériaux, jusqu'au moment où, ayant pris la résolution de faire de ces questions le sujet d'un travail inaugrural, je me mis à entreprendre l'exploration méthodique de la région lyonnaise.

Du reste, la distinction flatteuse dont mes confrères de la Sociélé botanique de Lyon m'ont honoré depuis sa fondation, a singulièrement facilité mes recherches; chargé en effet de la coordination et de la publication des Annales de cette So 
ciété, j’ai puisé dans les nombreux matériaux qui m'ont passé plusieurs fois sous les yeux, listes de plantes, comptes renclus d'herboristation (auxquelles j'ai du reste assisté la plupart du temps), une quantité de renseignements que je n'ai eu souvent qu’à colliger et à mettre en œuvre.

Les discussions soulevées dans le sein de cette association au sujet de l'influence exercée par le sol sur la végétation m'ont été aussi fort utileś, en m'aidant à me former une opinion sur la matière; je ne suis arrivé, je l'avoue, à adopter la théorie que je défends aujourd'hui, qu'à la suite de nombreux tâtonuements dus à une connaissance de plus en plus parfaite des faits. D'abord, le vif contraste qui me frappa à mon arrivée à Lyon, me fit admettre l'influence exclusivement chimique du sol; plus tard, la méditation de l'important ouvrage de Thurmann vint complètement modifier mes idées; enfin, une étude plus approfondie des causes diverses qui président à la distribution géographique des végétaux, les discussions soulevées au sein de la Socièté, les arguments apportés de part et d'autre, les voyages multipliés que je fis dans diverses contrées et qui m'ont permis de comparer les végétations d'un grand nombre de terrains différents, et surtout l'impulsion vraiment scientifique donnée aux recherches de géographie botanique dans le bassin du Rhône par mon excellent ami le Dr Saint-Lager, que je suis heureux de pouvoir remercier ici de ses obligeants conseils, m'ont fait revenir non pas à moll opinion première, trop absolue, mais à une conception qui me parait plus conforme aux faits, et qui attribue à l'influence chimique une part encore prépondérante. Il me paraît, en effet, bien établi aujourd'hui, par les observations précises de MM. Gontejean, Saint-Lager, etc., itendues à la plus grande 
partie de l'Est de la France et confirmées entièrement par mes recherches persomnelles dans les environs de Lyon et le Jura méridional, que si, dans ces régions, les terrains calcaires et siliceux ont une végétation si contrastante, cette différence est due surtout à la présence, à l'absence ou à la proportion relative des substances minérales, chaux, potasse, silice, etc.. qui entrent dans la composition du sol, et dans une plus faible mesure à ses propriétés physiques; mais j’admets difficilement des plantes exclusives, par la bonne raison qu'il n'y a pas généralement de sols exclusifs, et les dénominations de plantes calcicoles ou silicicoles que j'adopte, signifient dans mon esprit : plantes préférant les sols oí domine la chaux ct plantes préférant les sols ou dominent la potasse et la silicc. Je rejette donc l'expression créée par M. Contejean de plantes calcifuges, qui me semble prëjuger le ròle, encore si imparfaitement connu, des substances minérales, dans la nutrition des végètaux.

Le lecteur me pardonnera ces considèrations tout à fait personnelles: elles ont du moins l'avantage de montrer sous l'empire de ruelles préoccupations j’ai commencé ces recherches et dlans quel ordre diidées je les ai poursuivies. 


\title{
RECHERCHES
}

SUR I, A

\section{GEOGRAPHIE BOTANIQUE}

I) IS LA

\author{
FLORE LYONNAISE
}

\section{N TROD UGT I O N}

COUP DCEIL SUR LA TOPOGRAPHIE, LA GÉOLOGIE, LA CLIMATOLOGIE ET LA VÉGÉTATION DE LA RÉGION LYONNAISE

La contrée dont on décrit habituellement la vegétation sous le nom de Flore lyonnaise ${ }^{1}$ ne correspond pas à une région naturelle, nettement délimitée, comme la Bresse ou le Jura : elle ré sulte au contraire de la juxtaposition de parties plus ou moins étendues des régions naturelles qui entourent Lyon.

1 Quelques auteurs ont ètudiè sous le nom de Flore lyonnaise une circonscription fort étendue et mal délimilee, comprenant nou seulement la banlieue de Lyon, mais encore les monts du Lyonnais, du Beaujolais et même le Pilat, le Bugey et la GrandeCharireuse; dans ce travail, nous n'aurons en vue que les environs immédiats de Lyon, en nous servant, pour la commodite du discours, cles expressions de région lyonnaise, Flore lyonnaise, etc., comme synonymes, et avec le sens restreint que nous venons de leur donner. 
Si l'on prend, en effet, cette ville comme centre d'un champ d'études botaniques, il suffit de jeter les yeux sur une carte topo graphique et de faire quelques excursions en rayonnant suivant trois ou quatre directions de la rose des vents, pour reconnaître que la Flore lyonnaise embrasse des plaines, des vallées et des parties montagneuses se continuant insensiblement avec les montagnes et les plaines voisines.

A l'ouest, c'est le Lyonnais proprement dit, se rattachant par la nature du sol et par la Flore aux monts du Beaujolais, au Forez. et au plateau central; au nord-est, c'est le plateau de la Dombes, qui possède la même constitution du sol et la même végétation que la Bresse, dont il est la continuation; au sud-est s'étendent les plaines et les collines du Bas-Dauphiné, qu'il est difficile de séparer des autres parties plus méridionales de la même région; et enfin, au centre de la région lyonnaise, presque au point de rencontre des limites du Lyonnais, de la Dombes et du Dauphiné,se dresse le Mont-d'Or, massif montagneux, que la topographie, la composition des terrains, la nature de la végètation, isolent si complètement des trois autres régions au milieu desquelles il semble émerger.

Cependant, malgré les différences qui séparent chacune des parties constituantes de notre région lyonnaise, un examen de l'ensemble de leur Flore y fait découvrir des caractères communs dus à des faits curieux de dispersion des végétaux qui la composent.

La première de ces particularités remarquables, c'est l'existence sur les flancs de ces coteaux, bien exposés aux rayons du soleil, quelle que soit du reste leur nature géologique et quelle que soit aussi la région secondaire à laquelle ils appartiennent, de nombreuses espèces méridionales dont quelques-unes trouvent ici l'extrême limite de leur extension vers le nord; nous ne citons pour !e moment que l'Aphyllanthe, la Leuzée, le Genista horrida, diver's Cistes ou Hélianthèmes, l'Orchis papilionacea, etc.

D'autre part, la proximité des monts du Lyonnais et du Beaujolais, le voisinage des chaînes du Jura et du Dauphiné introduisent dans notre Flore d'autres espèces que l'étude de leur distribution géographique démontre lui être primitivement étrangères. 
C'est surtout par l'intermédiaire des cours d'eau qui descendent de ces régions montagneuses qu'ont lieu ces immigrations parfois temporaires, aboutissant d'autres fois à de véritables naturalisations.

C'est ainsi qu'en suivant l'Azergue et ses affluents, la Turdine et la Brevenne, qu'en descendant les vallées de l'Iseron et du Garon, les Geranium nodosum, Lychnis silvestris, Impatiens nolitangere, Epilobium obscurum, Montia rivularis, Digitalis purpurea, Cyslopteris fragilis, etc., arrivent jusque dans notre Bas-Lyonnais; et tandis que la Saône amène à Anse et dans nos îles de Royes, la Fritillaire ou le Tulipa precox, le Rhône entraîne avec lui, jusqu'aux portes de Lyon, des plantes jurassiques et alpines, telles que Linaria alpina, Centranthus angustifolius, Alsine verna, A. Jacquini, Sagina nodosa, Lepidinm petræum, Chlorocrepis staticifolia, etc,, les unes à existence tout à fait éphémère, les autres, comme le Lepidium et le Chlorocrepis, se reproduisant dans nos environs et pouvant être regardées maintenant comme spontanées.

La présence de ces espèces montagnardes dans nos plaines et nos vallées, leur végétation côte à côte avec les plantes méridionales et au milieu des espèces habituelles de la région, constituent le se cond caractère de la Flore lyonnaise et contribuent à lui donner cette richesse exceptionnelle que ne possèdent pas la plupart des autres Flores et qui en fait son principal attrait.

Indépendamment de ces caractères généraux, d'autres considérations peurent encore justifier le cadre que nous arons adopté dans ces études: c'est d'abord l'utilité qu'il y a pour le botaniste lyonnais à trourer réunis ensemble les tableaux de régétation des diverses régions qu'il est appelé à explorer chaque année; c'est en second lieu l'intérêt même qui s'attache ì la comparaison de ces Flores si différentes entre elles. Leur étude nous fournira l'occasion d'examiner l'importante question des rapports du sol avec la végétation et d'apporter des faits nouveaux à l'appui de la doctrine de l'influence chimique. 
Limites de la région lyonnaise. - La région lyonnaise telle que nous l'étudions dans ce travail est comprise dans un quadrilatère dont voici les limites:

Au nord, cette limite suit d'abord la vallée de l'Azergue depuis Lozanne jusqu'à son embouchure dans la Saône, puis une ligne fictive passant par Tréroux et Meximieux; à l'est, nous rencontrons une limite naturelle formée par l'Ain et la Bourbre; au sud, nous ne dépasserons pas la Verpillière, la vallèe de l'Ozon, Givors et le Mornantet; enfin, notre limite occidentale s'arrête au pied des monts du Lyonnais, suivant une ligne passant par Mornant, Vaugneray et Lozanne, notre point de départ.

Si cette délimitation parait, au premier abord, arbitraire, cependant on ne tardera pas à reconnaitre que l'Azergue, l'Ain, la Bourbre, l'Ozon, le Mornantet, circonscrivent assez bien notre région : l'Azergue la sépare du Beaujolais; l'Ain et la Bourbre, des montagnes calcaires du Bugey et du Dauphiné; l'Ozon et le Mornantet, de la Flore plus franchement méridionale des coteaux de Vienne, de Condrieu et de la vallée du Gier.

Mais à l'O., nos bas-plateaux et nos vallées se continuent presque insensiblement avec les monts de Saint-Bonnet, d'Iseron et de Riverie; cependant, tout en offrant la plus grande analogie dans leurs traits généraux, les végétations des bas-plateaux et des monts du Lyounais présentent entre elles des différences, dues aux rariations en altitude, suffisantes pour qu'il soit utile de les décrire séparément. Certaines espèces des bas-plateaux ne les quittent pas pour s'établir plus haut; par contre, d'autres, telles que Sambucus racemosa, Vaccinium Myrtillus, et les espèces montagnardes qui les accompagnent habituellement, ne lescendent jamais dans la zone inférieure. Dis reste, pour qu'il n'y ait pas de confusion à ce sujet, dans l'esprit du lecteur, en attendant un travail spécial sur la Flore des monts du Lyonnais et du Beaujolais, que nous espérons pouvoir publier plus tard, nous ajouterons en appendice une courte ètude comparative de leur végètation.

Un autre point où notre limite est tout à fait artificielle est la ligne O.-E. de Trévoux à Meximieux; car il est bien érident que 
ce que nous dirons de cette partie de la Dombes pourra s'appliquer à la Dombes entière et souvent à la généralité du plateau bressan, bien qu'on puisse trouver des différences dans la Flore de ses diverses parties suivant qu'on l'étudie au midi ou plus au nord, dans la zone à étangs ou sur les bords du plateau qui en sont généralement dépourvus; nous n'omettrons pas, du reste, l'indication de ces différences. Mais nous avons des raisons sérieuses $\dot{a}$ faire valoir pour limiter à Trévoux d'un côté et à Meximieux de l'autre, l'étude particulière que nous donnons aujourd'hui de cette partie de la Dombes; sa portion méridionale supporte, en effet, à son pourtour, une Flore spéciale, comprise assez exactement dans les limites données plus haut et qui offre la plus grande analogie avec celle des autres coteaux du Rhône et de la Saône avoisinant Lyon et de plus avec celle du Mont-d'Or, des collines du Revermont et de Crémieux.

DIVISIONS. - TOPOGRAPHIE. - GÉOLOGIE ET VÉGÉTATION

EN GÉNÉRAL

La région Iyonnaise ainsi limitée, se divise en deux parties bien distinctes, l'une à l'E. de Lyon, l'autre à l'O., séparées par la dépression N.-S. dans laquelle coule la Saône d'abord, puis le Rhône, à partir de leur confluent, qui a lieu à l'extrémité méridionale de la citè lyonnaise.

La partie occidentale se subdivise à son tour en deux régions secondaires : la première comprenant les formations gneissiques et granitiques des vallées et bas-plateaux du Lyonnais; la seconde, située au N. de la précédente, constituée par le massif jurassique du Mont-d'Or.

I. Lyonnais granitique. - Les roches mètamorphiques, gneiss, micaschistes, coupées de distance en distance par des filons plus ou moins étendus de granites, composent la plus grande partie du sol de notre première région secondare; elles forment l'ossature de nos bas coteaux, sortes de rides qui se détachent plus ou moins 
obliquement des monts du Lyonnais ; ces roches ne dépassent guère les limites de notre première région du côté de l'orient; on ne les rencontre qu'accidentellement sur les rives gauches de la Saône et du Rhône, à Rochetaillée, en face de l'Ile-Barbe, sous la CroixRousse, et à partir de Saint-Symphorien d'Ozon en se dirigeant sur Vienne; notons aussi, pour mémoire, le petit îlot tout à fait isolé de Chamagnieu (Isère); bien que ces deux derniers points soient situés sur les limites de notre région, un peu en dehors d'elles, nous les citerons quelquefois à cause de certaines particularités intéressantes de leur végétation. Revenant au Lyonnais proprement dit, l'étude de la Flore de ces terrains exclusivement siliceux nous permettra de constater que la présence des Sarothammus scopa.rius, Teesdalia nudicaulis, Jasione sp., Omithopus perpusillus, Arnoseris minima, Anarrhinum bellidifolium, Galeopsis dubia, des Aira et Vulpia divers, l'abondance des Fougères, des Mousses et des Lichens et parmi ces cryptogames, l'Asplenizm septentrionale, les divers Polytrichs, les Racomitrium canes. cens, Rhizocarpon (Lecidea) geographicum, caractérisent notre première région naturelle secondaire.

Deux points se distinguent du reste de la partie située à l'O. de la vallée du Rhône et de la Saône, par la nature du sol et de leur Flore, ce sont les coteaux les plus rapprochés de cette vallée et le Mont-d'Or lyonnais.

II. Le Mont-d'0r, massif jurassique se rattachant aux formations analogues du Bas-Beaujolais par les îlots de Dardilly et de Civrieux, est composé à sa base et jusqu’à une certaine hauteur dans sa partie occidentale, de roches à éléments siliceux prédominants (grès, calcaire siliceux du trias, etc.), dont la végétation ne diffẻre pas de celle du Lyonnais granitique; au-dessus, ce sont les mar nes et les calcaires des étages liasien et bajocien, supportant une Flore bien distincte, caractérisée par le Buis, les Coronilla Ememes, Daphne Laureola, Geranium sanguineum, Lithospermum purpureo-crinleum, les Orchis et Ophrys si nombreux, les Grimmia crinita, Placodium callopismum, etc., et l'absence absolue du Sarothamne et des autres espèces citées plus haut comme par- 
ticulières à notre première région secondaire. Cependant l'étude approfondie que nous ferons des différentes parties de cette région nous montrera que dans certains points le calcaire devient très siliceıx, au niveau du Ciret par exemple, et qu'aussitôt, fait digne de remarque, on voit la Flore changer et des espèces réputées silicicoles y élire domicile.

III. Coteaux du Rhône. - Les coteaux les plus voisins du Rhône et de la Saône se distinguent du reste de la partie occidentale de la région lyonnaise en ce que leur sous-sol de gneiss et de granite est recourert par une épaisseur plus ou moins grande de dépôts alluviens, conglomérats, boue glaciaire, lehm, à composition minéralogique et à végétation très variables.

Ces alluvions anciennes et ces dépôts glaciaires se retrouvant dans chacune des autres régions secondaires, nous réunirons leur étude en un même paragraphe. Cependant nous ferons remar. quer dès maintenant que ces alluvions anciennes se sont étendues assez loin sur les bas plateaux du Lyonnais; on les retrouve en effet jusqu'à Charbonnières, Saint-Genis les Ollières, Chaponost, Montagny; une ligne continue passant par ces villages représente assez exactement leur extrême limite occidentale. Dans une partie de leur étendue, ces alluvions sont constituées principalement par des quartzites et forment un sol pauvre en carbonate de chaux; il arrive même quelquefois que celui-ci manque complètement, et leur végétation se rapproche alors beaucoup de celle de la région granitique ${ }^{1}$ proprement dite : il en est ainsi dans les environs de Dardilly, Charbonnières, Tassin, où l'on peut voir le Sarothamne et lạ Bruyère croître vigoureusement et abondamment sur les alluvions. Mais il n'en est pas de même dans les parties plus rapprochées du Rhône, c'est-à-dire sur les coteaux de Sainte-Foy lès Lyon, SaintGenis-Laval, Millery, sur les bords du plateau de la Dombes, depuis Trévoux jusqu'à Meximieux, dans certaines parties des Balmes viennoises, telles que les coteaux de Saint-Fons à Sérézin ; ici ces

1 Il est bien évident que, par ce mot de région granitique, nous entendons non seulement les terrains formés par des granites, mais aussi ceux constitués par des gneiss, micaschistes, porphyres, etc. et d'autres roches silicatées. 
dépôts récents sont formés soit par de la boue glaciaire riche en cailloux jurassiques (Sainte-Foy, Saint-Genis, Millery, etc.), soit par des conglomérats de cailloux quartzeux, mais réunis en poudingues par un ciment calcaire (Sainte-Foy, Beaunant, Sathonay, la Pape, etc.), soit par des mollasses calcaires, comme à Saint-Fons et Feysin; la végétation est alors manifestement calcicole, à $C o$ ronilla Emerus, Aster Amellus, Inula montana, Ononis Columnx, Rubia peregrina, etc., et on n'y rencontre le Sarothamne et d'autres plantes silicicoles telles que les Jasiones, l'Anarrhinum, etc., que dans les parties très perméables, exposées depuis longtemps aux influences atmosphériques, dont le carbonate de chaux a été entraîné dans les couches les plus profondes par une sorte de lixiviation due à l'infiltration des eaux pluviales, et qui sont devenues ainsi accidentellement et tout à fait localement des sols siliceux.

IV. Bas-Dauphiné et Dombes. - La partie de la région lyonnaise située à l'E. de la vallée N.-S. du Rhône et de la Saône est elle-même subdivisèe en deux régions secondaires par la vallée du Rhône, qui au-dessus de Lyou, se dirige à angle droit de la direction précédente en cuulant de l'E. à l'O.

$A u N$. et au S. de cette ligne, s'élèvent deux plateaux, le plateau bressan et le Bas-Dauphiné, auxquels on peut distinguer des bords et une partie centrale; les bords appartiennent à la région botanique secondaire des coteaux du Rhône et de la Saône dont nous venons de voir la communauté de nature de sol et de végétation. Quant aux parties centrales, elles ont une importance bien inégale : celle du Bas-Dauphiné se confond en partie arec ses bords; il faut aller vers les prairies des bords de la Bour bre, les marais des Avenières et la région mollassique dite des Terres froides, situées en dehors de nos limites pour trouver des faits intéressants de géographie botanique. Mais il n'en est pas ainsi du plateau de la Dombes situe au N. de notre ligne de partage E.-O. Nous trouvons là, dans sa partie centrale, un sol spécial, le terrain à étangs, supportant une végétation particulière qu'on peut qualifier de silicicole si l'on se place au point de vue de l'in. 
fluence de la composition minéralogique du sol, mais qui est surtout hygrophile, comme l'indiquent : Myosurus minimus, Lotus dif-fusus, Lythrum hyssopifolium, Corrigiola littoralis, Peplis, Montia, Centunculus minimus, Lindernia pyxidaria. Litto. rella lacustris, etc.; notons aussi la fréquence, dans les terrains plus secs, des Sarothamne, Gypsophila muralis, Spergula, Hypericum pulchrum, Ulex, Arnoseris minima, Galeopsis dubia, qu'on retrouve pour la plupart dans le Lyonnais granitique.

Enfin, pour terminer l'énumération des diverses parties qui com. posent la Flore lyonnaise, il nous reste à mentionner les alluvions des bords du Rhône et de la Saône, dont la végétation a un cacliet spécial; nous l'étudierons en même temps que celle des coteaux aux pieds desquels ces alluvions s'étendent, les plantes qui pour raient la caractériser passant souvent de l'une à l'autre de ces formations.

T'el est, vu dans son ensemble, le vaste champ que nous allons explorer en détail. Après en aroir étudiè chaque partie dans sa topographie, la nature de son sol et la caractéristique de sa végé tation, nous devrons ensuite comparer ces diverses régions secon-daires l'abord entre elles, puis avec les régions naturelles voisines.

COMPARAISON EES RE GIONS SEGONDAIIES

Nous avons déjà les éléments de ce travail de comparaison dans le courtes listes d'espéces que nous avons données comme caractéristiques de chacune de ces réģions naturelles; elles suffisent, en effet, pour montrer quelle similitude il y a entre la Flore des basplateaux lyonnais et celles de la Dombes d'une part, entre la végétation du Mont-d'Or lyonnais et celle des coteaux du Rhône et de la Saône d'autre part; les deux premières ayant comme plantes caractéristiques communes : Sarothamne, Gypsophila muralis, les Spergules, Hypericum pulchrum, Arnoseris minima, Galeopsis clubia; les secondes : Coronilla Enncrus, Rubia pere- 
grina, Lithospermum purpureo-cceruleum, Aster Amellus, Inula montana, les Orchis et Ophrys à espèces et individus si nombreux dans les régions calcaires.

Le travail de comparaison des régions secondaires qui constituent la Flore lyonnaise avec les régions voisines, le Jura et le Forez, nous offre un intérêt non moins grand: nous voyons d'abord la partie montagneuse des Bas-Plateaux de la région granitique rapprochée des monts du Lyonnais, du Forez et du Pilat, par une série d'espèces caractéristiques de la Flore du plateau central ; ce sont, par exemple: Ranunculus hederacens, Dianthus deltoicles, Rubus glandulosus, Montia rivularis, Senecio adoniclifolius, Digitalis purpurea, Myosotis lutea, Asplenium septentrionale, etc.; puis, la région à étangs de la Dombes et les points argileux du Lyonnais granitique qui présentent la plus grande analogie avec la plaine du Forez par la présence de part et d'autre des Stellaria uliginosa, Lythrum hyssopifolium, Montia minor, Corrigiola littoralis, Centunculus minimus, Littorella lacustris, Scirpus setacens, etc.

De même si l'on compare les listes que nous donnons des plantes caractéristiques des régions du Mont-d'Or lyonnais et des coteaux du Rhône, avec une énumération des plantes qui croissent habituellement dans les monts de Crémieux, du Bugey ou du Jura méridional, on pourra y relever des espèces communes telles que: Geranium sanguineum, Coronilla Enerus, Peucedanum Cervaria, Seseli coloratum, Lithospermum purpureo-caruleum, Galium corrudcefolium, Rubia peregrina, Asler Amellus, Inula montana, Campanula Medium, Thesium divaricatum, Buxus sempervirens, etc.

Influence de la composition chimique du sol. - Tous ces exemples d'analogie de végétation correspondant précisément a des analogies dans la composition chimique des sols des régions, sont déjà des arguments nombreux et puissants en faveur de l'influence chimique du sol sur la régétation. Mais, dans le chapitre que nous consacrerons à l'examen de cette question, nous en apporterons d'autres plus significatifs encore. Si l'ètude compa- 
rative des régions nous a fourni les éléments de la comparaison en grund, l'examen minutieux de certains points particuliers qui, de prime abord, paraissent des exceptions, nous fournira des preuves tirées de la comparaison ou des contrastes en petit, comme les appelle Thurmann; en effet, dans chacune des régions de notre Flore lyonnaise on peut trouver des points limités dont la végétation diffère complètement de celle'qui caractérise l'ensemble de la région; or, nous montrerons que ces changements dans la végétation sont toujours accompagnés de modifications correspondantes dans la composition minéralogique du sol et que, par conséquent, ce qui paraissait l'exception n'est que la confirmation de la théorie.

Influence physique du sol. - Si l'influence prépondérante de la composition du sol sur la dispersion des végétaux ressort d'une façon évidente de la comparaison des Flores qui caractérisent chaque partie de la région lyonnaise, l'étude la plus superficielle fait voir que l'ètat physique des terrains exerce aussi une certaine influence, mais une influence secondaire. En effet, dans chacune de nos régions, nous trouvons des sols meubles (eugéogènes de Thurmann), à espèces psammiques ou psammophiles, et des sols compactes (dysgéogènes), à espèces péliques, les unes et les autres pouvant à leur tour préférer un sol humide ou les terrains secs, être par conséquent, pour employer une terminologie uniforme, hygro-philes ou xérophiles; sans compter les sols mixtes, pélopsammogènes, produisant des espèces pélopsammophiles, hémipsammiques, hémipéliques, etc. Et ce n'est pas une des preuves les moins fortes en faveur de l'influence chimique que de voir, non pas les mêmes espèces, mais le mème ensemble de végétation, à la fois sur les sols sableux et argileux du Lyonnais, sur les terrains argileux et sableux de la Dombes, ou sur les terrains péliques du Mont-d'Or, et les sables calcaires des coteaux du Rhône.

Mais il y a, outre les espèces adhérentes, une série de plantes indifférentes i la composition chimique du sol et qui croissent plus spécialement sur les rochers, dans les sables ou les graviers, les argiles ou les marnes, les prairies naturelles, les bois ou les terres 
cultivées, etc.; à ce point de vue se rattache l'étude des associations végétales des haies, des fossés, des prairies, etc.

Sous le nom de Flore des Tourbières, nous consacrerons un paragraphe spécial ${ }^{1}$ aux prairies marécageuses, si répandues dans les vallées et sur les bas-plateaux du Lyonnais; aux marais tourbeux des Échets, de Sainte-Croix, dans la Dombes; à ceux de Décines, de Frontonas, des bords de la Bourbre, dans le Bas-Dauphiné : nous comparerons leur Flore arec celle des marais tourbeux des monts du Lyonnais, du Forez et des tourbières dı Jura.

Que ces marais tourbeux soient situés dans les régions calcaires ou dans les régions siliceuses, nous constateronsque leur végétation présente la plus grande analogie; mais, loin d'être un argument contraire il'influence chimique, cette analogie est une preuve de plus, car les espèces qui habitent ces stations sont en génèral des plantes silicicoles, et le sol des Tourbières ou marais tourbeux des régions calcaires est toujours dépourru de carbonate de chaux, ainsi que le montrent les analyses.

Influence de l'exposition. - Un autre facteur important dont il faut tenir compte dans la dispersion des plantes, est l'exposition; nouscompareronsà ce point de vue les régions de notre Flore, soit dans leurs détails, soit dans leur ensemble, et ce travail nous permettra de voir quelle est l'influence de l'exposition dans la composition du tapis végétal des vallées du Lyonnais, du massif du Mont-d'Or et des coteaux du Rhône; les vallées du Lyonnais, di . rigées génćralement de l'O. à l'E., ont, dans une grande partie do leurs cours, deux versants dont la végétation est bien distincte; le versant $\mathrm{N}$. ou N.-O. du Mont-d'Or n'a aucune des espèces de cette Flore méridionale si intéressante qui couvre les pentes exposées au S. ou au S.-E.; de même la Flore des coteaux du Rhôre, et principalement de la partie qui s'étend de Saint-Clair à Meximieux et qui regarde directement au midi, est caractérisée par des espèces méridionales qui trouvent dans cette exposition privilégiée

1 Revision dun premier travail publiè par nous dans le Bull. de la Suc. bot. de France, t. XXI, 1874, session de Gap, 1). xxxv. 
soit un abri contre les gelées par les vents du nord, soit la chaleur nécessaire pour se développer et mûrir leurs graines. L'étude de la dispersion des plus remarquables de ces espèces nous mon-trera qu'un certain nombre d'entre elles, comme Cistus salvicefolius, Helianthemum salicifolium, Genista horrida, Leuzea conifera, Aphyllanthes monspeliensis, Orchis mbra, etc., ne remontent pas plus haut dans la vallée de la Saône.

Influence de l'altitude. - Les variations altitudinales se font, dans les environs immédiats de Lyon, entre des limites si étroites qu'elles ne déterminent que de légères modifications dans la Flore. $\mathrm{Si}$, partant des bords du Rhône, dont la cote à Lyon est à $160 \mathrm{~m}$., nous nous élevons successivement, nous rencontrons d'abord les collines du Bas-Dauphiné dont l'altitude oscille entre 200 et $220 \mathrm{~m}$., le plateau de la Dombes et les bas-plateaux du Lyonnais, dont l'altitude moyenne est de $300 \mathrm{~m}$., et enfin le Mont-d'Or, dont les plus hauts sommets dépassent' à peine $620 \mathrm{~m} .{ }^{1}$. Il faut sortir de

- nos limites pour rencontrer dans les monts du Lyonnais une zone se maintenant entre 800 et $900 \mathrm{~m}$., n'atteignant 1,000 m. qu'au mont Boucivre, au-dessus de Tarare. En nous basant surtout sur les données fournies par l'agriculture et par la comparaison arec les zones établies dans les régions voisines, nous distinguerons dans notre région lyonnaises les trois zones de végétation suivantes :

$1^{\circ}$ Zone des vallées basses : de 160 à $260 \mathrm{~m}$.; altitude moyenne, $200 \mathrm{~m}$.; température moyenne $=12^{\circ} ;$ moissons du $1^{\text {er }}$ au 8 juillet. C'est la zone des vallées du Rhône et de la Saône, des vallées et des collines du Bas-Dauphiné;

$2^{\circ}$ Zone de la Vigne et du Chälaignier : Bas-plateaux du Lyonnais, Mont-d'Or et plateau de la Dombes; altitude de 260 à 560 m.; température moyenne $=11^{\circ} ;$ moissons du $8-15$ juillet ;

$3^{\circ}$ Zone des Pins : monts du Lyonnais, Saint-Bonnet, Iseron, Duerne, etc.; altitude, 560 à $900 \mathrm{~m}$.; température moyenne =$10^{\circ}$; moissons vers le 20 juillet.

¿ MonŁ Toux, 612; Mont-Verdun, 625, d'après l'ètal-major. 
Influence du climat. --- Nous croyons devoir accompagner l'exposé de nos recherches phytostatiques d'un court aperçu de la climatologie générale de la région lyonnaise. Notre intention était primitivement de consacrer à chaque région secondaire un paragraphe donnant les températures moyennes et extrêmes, l'intensité de la radiation solaire, la température des sources, etc. Mais le peu d'observations qu'il nous a été donné de recueillir ne nous permet pas, pour le moment du moins, d'entrer dans une étude si complète; nous nous bormerons à un exposé général et, pour beau. coup de points, à indiquer les recherches à faire, en émettant le vœu que des observations météorologiques aient lieu dans un plus grand nombre de localités de notre région et qu'on n'oublie pas les mesures actinométriques, les variationes, de la température du sol, etc., dont nous ne possédons encore aucune observation locale et qui sont cependant indispensables pour établir sur des données sérieuses la climatologie végétale d'une contrée.

Après avoir exposé l'état actuel de la végétation de la Flore lyomaise, nous compléterons ce tableau par l'examen de ses modifications dans la suite des temps géologiques et historiques. Nous nous bornerons, pour cette étude dans les temps géologiques, à un court résumé des travaux de nos paléontologistes sur les végètations houillères de Saint-Étienue, Ternay, Valsonnne Sainte-Foyl'Argentière, etc., des Flores kimméridgiennes de Cerin, Morestel, de la Flore pliocène de Meximieux, qui suffiront pour nous donner ure idée des climats diver's et des végétations qui se sont succédé dans notre région; mais pour les modifications survenues depuis la période historique, nous examinerons quelle est la part qu'il faut faire aux changements climatologiques et à l'influence directe de l'homme; nous montrerons que par suite des défrichements et des cultures, le climat s'est modifie, de froid et humide est devenu plus sec et plus chaud, que la végétation a subi des modifications corrélatives par la disparition ou la diminution du nombre des es péces silvicoles et l'augmentation des formes plus méridionales; que les ensemencements par les graines étrangères, les transports 
par les bateaux, surtout par les chemins de fer, el quelquefois l'action directe et volontaire même de l'homme ont introduit dans notre Flore des espèces étrangères, les unes, trop méridionales, ne pouvant vivre sous notre climat que temporairement, végétaux $a d-$ ventifs, auxquels nous consacrerons un chapitre spécial. ; les autres, principalement d'origine américaine, que la similitude des climats ou le milieu aquatique dans lequel elles vivent, a fait naturaliser avec plus de facilité, comme les Erigeron canadlensis, Solidago, Aster, Elodea canadensis, etc.; nous verrons enfin que si,par le fait des cultures, des espèces rares de la Flore, comme le Ciste, le Genista homida, etc., sont en voie de disparition, nous assistons, de nos jours, à des envahissements manifestes d'espèces terrestres, telles que Barkhausia setosa, Plerotheca nemausensis. ces modifications actuelles de la Flore que le grand nombre des observateurs a permis de suivre et d'enregistrer avec soin, sont des exemples indéniables des modifications analogues qui ont dû se produire antérieurement et dont les botanistes anciens ne nous ont laissé malheureusement aucune preuve.

En résumé, notre travail comprendra les parties suivantes :

DISTRIBUTION GÉGRAPIIQUE DES VÉGÉTAX DANS L'ESPACE VÉGETATION AGTUELLE DE LA RÉGION LYONNAISE

A. Études particulières des régions:

Vallees et bas-plateaux du Lyonnais. . Premiére partie.

Coteaux et Vallées du Rhòne et de la Saône. DEUXiÈme PARTiE.

Mont-d'Or. . . . . . . . . . Troisième partie.

Plateau de la Dombes. . . . . . Quatulìme partie.

L. Étude comparée des réfions. . . CINQUIEME Pante.

I. Influence de la composition minéralogique du sol :

1. Compuraison du Bas-Lyonnais, de la Dombes et du Forez

20 Comparaison du Mont.d.Or, des coteaux du Rhòne et du Jura

3o Faits anormaux de dispersion: Plantes calcicoles des régio s siliceuses; - Plantes silicicoles des régions calcaires. 
II. Influence de l'exposition. Flore méridionale iu Lyonnais.

III. Influence du climat, de l'altitude, etc., et Climatologie de la region lyonnaise. . . . . . . . SIXIÈ PARTIE.

\section{II}

DISTRIBUTIONS DES VÉgETAUX DANS LE TEMPS MODIFICATIONS DE IA FLORE DU LYONNAIS

A. Dans les temps gëologiques. . Septième Partie.

B. Depuis la periode historique. . . - - -

Changements climatériques. Inlluence des déboisements. Plantes en voie de disparition.

Plantes envahissantes.

Flore rudérale; Fl. des cultures; Fl. adventice. 


\title{
PREMIERE PARTIE
}

\author{
VALLEESS ET BAS-PLATEAUX DU LYONNAIS
}

\section{GHAPITRE PREMIER}

TOPOGRAPHIE

La région située à l'ouest de Lyon peut être considérée comme formée par un massif montagneux dirigé dans son ensemble du nord au sud, ce sont les monts du Lyonnais, et par des bas-plateaux qui s'en détachent sous un angle plus ou moins grand.

La partie la plus orientale des monts du Lyonnais est constituée par deux chaînes de montagnes : l'une septentrionale, dirigée du N.-E. au S.-O., de Lentilly à Iseron, peut être appelée chaine de Saint-Bonnet; l'autre, qui lui fait suite, et qui s'étend jusqu'aux environs de Riverie, mais avec une orientation directement N.-S., a reçu de Fournet le nom de chaine ou côte Saint-André'.

De ces deux chaînes primordiales se détachent des chaînons obliques qui se dirigent tous vers le N.-E., en s'abaissant graduelle ment vers la Saône et le Rhône; leur orientation $0.40^{\circ} \mathrm{S}$. à E. $40^{\circ} \mathrm{N}$., est parallèle à la chaîne du Pilat. Leur disposition a été

1 Voy. Fournet : Etudes pour servir à la Géographie physique et à la Géologie drune partie du bassin du Rhône. (Ann. de la Soc. d'Agricult. de Lyon, 1838, t I, p. 4 et seq.) 
bien décrite par Fournet ${ }^{1}$, à qui nous empruntons une partie des détails qui suivent.

Le $1^{\text {er }}$ de ces chainons ou axe du Mont-d'Or, part de Mercruy ( $570^{\mathrm{m}}$ à l'extrémité de la chaîne d'Iseron) “ passe par la Tour-deSalvagny $\left(350^{\mathrm{m}}\right)$, Limonest $\left(400^{\mathrm{m}}\right)$, les sommets du Mont-d'Or $\left(600^{\mathrm{m}}\right)$ et s'abaisse dans les environs d'Albigny $\left(300^{\mathrm{m}}\right)$; " nous verrons, lorsque nous parlerons de la Dombes, que Fournet regardait cet axe comme se prolongeant sous le plateau bressan jusqu'au pied du Revermont. Ce chaînon sépare le bassin de l'Azergue de ceux de l'Iseron et du ruisseau de Charbonnières, son principal affluent.

Le $2^{\circ}$ chaînon ou " axe de Francheville, part du Pied-Froid $\left(704^{\mathrm{m}}\right)$, près d'Iseron, passe par Brindas $\left(320^{\mathrm{m}}\right)$, Francheville $\left(230^{\mathrm{m}}\right)$, aboutit à Pierre-Scize et à la Croix-Rousse; " sa partie occidentale sert de ligne de séparation aux deux bassins de l'Iseron et du Garon.

Le 3 e ou " axe de Riverie, se déprime subitement derrière Mornant, puis forme une légère ride terminée au-dessus du Rhòne près de Vernaison et Irigny. " Il se comporte de même que l'axe précédent et, dans sa partie occidentale, sépare les bassins du Garon et du Mornantet.

Il résulte de cette disposition que les vallées de l'Iseron, du $\mathrm{Ga}$ ron et de leurs affluents les ruisseaux de Gour, Pleine-Serve, Méginant, Mercier, Chalandresse, Soucieu, etc., sont toutes dirigées du S.-O. au N.-E. dans la première partie de leur parcours ; puis, leurs eaux prennent une direction plus ou moins perpendiculaireà la précédente avant de se jeter dans le Rhône. Du reste, dans cette dernière partie, les vallées du Lyonnais présentent des diffèrences non seulement dans leur direction, mais encore dans la nature du sol et de leur végétation; nous reviendrons plus loin sur ces particularités.

Le squelette de cette région ainsi exposé, étudions avec plus de détails les vallées les plus importantes et les plateaux qui les ś́parent.

1 Op. cit., p. 16. 
Nous décrirons, en allant du N. au S., les vallées du Charbonnières et de ses affluents le Ratier et les Ribes, les vallées de l'Isère et du Garon.

Le Charbonnières ${ }^{1}$ prend naissance entre Marcy et la Tourde-Salvagny, par la réunion des deux ruisseaux du Gour et de la Grande-Serve, alignés suivant l'axe du Mont-d'Or. A partir de ce confluent, le ruisseau de Charbonnières court perpendiculairement à cette direction, reçoit la source minérale de Charbonnières, le ruisseau de Dardilly, puis sous Tassin, le Ratier, et, après avoir passé sous le Pont-d'Alaï, vient se jeter dans l'Iseron un peu audessus de Francheville.

Dans sa partie supérieure, le lit du Charbonnières est creusé dans le granite; au-dessous du village de Charbonnières, la vallée s'élargit et les rocs font place à des prairies qui la bordent presque continuellement jusqu’à son embouchure; de temps en temps cependant le vallon se resserre entre les gneiss, sous Tassin et vers le Pont-d'Alaï, par exemple.

Le Ratier, qui se jette dans le ruisseau de Charbonnières, sous le village de Tassin, naît de la chaine d'Iseron, au-dessus de Pollionnay, reçoit sur sa droite le ruisseau Mercier, puis court, au fond d'une vallée très resserrée entre deux pentes abruptes de gneiss et de granite; un peu au-dessus du point où il reçoit le ruisseau des Ribes, la vallè s'élargit, et des prairies, souvent marécageuses, règnent sur ses bords jusqu'au pont de Tassin.

Le ruisseau des Ribes naît sous le hameau de la Brosse (commune de Marcy), reçoit le ruisseau de Quincieux, qui prend naissance près du précédent, mais un peu plus au sud; leur vallée est creusée d'abord dans le granite et le gneiss, puis dans les alluvions glaciaires ; cependant en arrivant vers son embouchure, le gneiss

1 Cf. A. Perrey. Etudes hydrauliques sur les ruisseaux de la Brevenne, l'Orgeol, l'Iseron, le Charbonnières, le Garon, le Mornantet et la Coise. (Ann. Soc. d'Agric. de Lyon, 2e série, t. V, 1853, p. 446, 473 et seq.) 
reparaît sur ses bords; dans presque toute leur longueur, les ruisseaux des Ribes et de Quincieux coulent au milieu de prairies fort peu développées en largeur.

L'Iseron ${ }^{1}$ prend sa source près du village dı même nom, à $761^{\mathrm{m}}$ d'altitude; jusque auprès de Francheville, il est dirigé suivant l'axe N.-E., et son lit est creusé dans le granite et le gneiss; un peu avant de recevoir le Charbonnières, au nord de Francheville, sa direction change et suit le second axe, N.-O.; après avoir franchi le défilé granitique que surplombent les ruines de l'ancien château de Francheville, l'Iseron parcourt une vallée plus large creusée dans les alluvions glaciaires; des prairies le bordent dans la plus grande partie de son cours, sauf dans les points où les gneiss et les granites se rapprochent, comme sous Craponne, vers le PontJambon, sous Bel-Air, à Francheville et en arrivant à Oullins; au-dessous de ce village, l'Iseron se jette dans le Rhône.

Le Garon ${ }^{2}$ prend naissance sur la commune d'Iseron, à $657 \mathrm{~m}$. d'altitude; il reçoit d'ahord les ruisseaux de Chassagne, d'Arquillières et du Villars, vers le village de Thurins, où la vallèe du Garon quitte le gneiss, pour s'engager dans ce large filon de granite qui s'étend jusqu'à Saint-Genis-Laral. A partir de Thurins jusqu'au confluent de la Chalandresse, le Garon suit l'axe N.-E., puis l'axe N.-O., de ce dernier point jusqu'à Brignais; dans ce dernier trajet, son lit creusé dans le granite, reçoit divers ruisseaux, de peu d'importance, sauf un, sur sa droite, le Furon; vers le moulin Barelle, ou du Barail, reparaissent les gneiss et les autres roches métamorphiques; la vallée s'èlargit en prairies étendues qui se continuent jusqu’à Brignais.

Au-dessous de ce village, jusqu'à l'embouchure du Garon dans le Rhône, près de Givors, le lit de cette rivière est creusé dans une plaine alluviale, dirigée du N. au S., assez large et occupée par des prairies ou des cultures; cette vallée N.-S. sépare nettement les plateaux siliceux du Lyonnais, des coteaux du Rhòne à sol formé

1 Voy. A. Perrey. Op. cit., p $46 \%$.

2 Voy. A. Perrey. Op. cit., p. $4 i$. 
superficiellement par des terrains de transport ${ }^{1}$; dans cette dernière partie de son cours, le Garon reçoit sur sa droite une série de ruisseaux qui descendent tous parallèlement, avec l'orientation gènérale O.-E., des plateaux Lyonnais : ce sont le Chéron, le Caranona, et le Mornantet avec son affluent le ruisseau du Brullon,

Le principal de ces affluents, le Mornantet, qu'on pourrait, du reste, considérer comme un ruisseall distinct qui se serait réuri au Garon un peu au-dessus de leur confluent dans le Rhône, a sa source sous Saint-André, à $613 \mathrm{~m}$. d'altitude; son lit est creusé dans le granite et le gneiss, et à sa partie inférieure, dans les alluvions qui font suite à celles du Garon. Le Mornantet forme la limite méridionale de la partié de la région du Lyonnais que nous étudions dans ce travail; il sépare, en effet, les terrains granitiques de Chassagny et des plateaux du Lyonnais, des formations houillières du bois de Montrond et de la vallée du Gier.

A ces bassins principaux de l'Iseron et du Garon nous joindrons les vallées du ruisseau de Rochecardon et du ruisseau des Planches; le premier est formé par la reunion des ruisseaux de Limonest et de Saint-André avec celui qui prend naissance sous SaintFortunat; le second naît par deux branchés qui entourent le plateau de Dardilly et reçoit successivement les ruisseaux d'Écully et de Chaleins; tous deux se jettent dans la Saône à Vaise ou un peu au-dessus. Bien qu'elles ne proviennent pas des plateaux du Lyonnais, mais plutòt des plateaux du Mont-d'Or, ces vallées offrent la plus grande analogie avec les premières, par leur disposition, la nature du sol dans lequel elles sont établies tt leur végétation; en effet, de même que les vallées du bassin de l'Iseron et dı Garon, celles qui entourent la base du Mont-d'Or sont creusées, dans la première partie de leur cours, dans les gneiss et le granite; en se rapprochant de leur embouchure, elles prennent une directio:1 N.-O.-S.-E, et les plateaux qu'elles sèparent, sont recouverts pa' des terrains de transports, lehm et alluvions, devenant de plus

1 Ce qui explique pourquoi les allıvions de cette dernière partie du Garon sont bien plus lertiles que les alluvions de l'Iseron. 
en plus riches en carbonate de chaux à mesure qu'on se rapproche de Lyon et présentant alors une végétation analogue à celle des coteaux du Rhône.

En résumé, toutes ces vallées offrent entre elles les plus grandes analogies; ce sont toujours des vallons plus ou moins profonds, à bords généralement escarpés, incultes, boisés ou non, séparant des plateaux ordinairement cultivés, quelquefois couverts de bois ou à l'état de bruyères. Lorsque le fond de la vallée présente quelque largeur, il est occupé par des prairies, devenant en maints endroits marécageuses ou tourbeuses par suite du défaut d'écoulement des eaux.

Ce sont, en allant du N. au S. :

$1^{\circ}$ Le plateau d'Ecully, triangulaire, ì sommet dirigé vers le N., limité par le ruisseau de Limonest au N.-E., et le ruisseau des Planches à l'O. et au S.;

$2^{\circ}$ Le plateau de Dardilly, aussi triangulaire, mais à sommet méridional, compris entre le ruisseau des Planches et celui de Serres ;

3. Le plateau de la Tour-de-Salvagny, limité par les ruisseaux des Planches et de Charbonnières; il se prolonge au N. par le plateau de Dommartin vers le petit massif jurassique de Civrieux; au S., il s'abaisse graduellement et devient la plaine de Tassin et de la Demi-Lune;

$4^{\circ}$ Plateau de Marcy -l'Étoile et de l'Aigua, compris entre le ruisseau de Charbonnières et le ruisseau des Ribes;

$5^{\circ}$ Plateau de Méginant, s'étendant entre les ruisseaux des Ribes et de Quincieu ;

$6^{\circ}$ Plateau de Saint-Genis les Ollières, entre le ruisseau des Ribes et celui du Ratier ;

$7^{\circ}$ Plateau de Craponne allongé entre le Ratier au N., l'Iseron, au S. et terminé à l'E. par le Charbonnières; 
$8^{\circ}$ Plateau de Brindas et Chaponost, vaste triangle à sommet dirigé vers l'O., dont la base est représentée par la vallée de Beaunant, les côtés par l'Iseron au N., le Garon au midi ;

$9^{\circ}$ Coteaux d'Orliẻnas, de Taluyers, de Chassagny (alt.300-400 ${ }^{\mathrm{m}}$ ), compris entre le Garon, ses affluents de rive droite et le Mornantet.

Les trois premiers plateaux se rattachent aux pentes du Montd'Or, les autres aux contreforts des monts du Lyonnais, dont ils ne sont que la continuation suivant les axes N.-E. que nous avons indiqués plus haut; cette relation apparaît encore mieux dans les coteaux plus rapprochés de la chaîne lyonnaise, que nous avons laissés, à dessein, en dehors de l'énumération précédènte, et qui sont :

$1^{\circ}$ Le coteau du Poirier (alt. 300-400), situé entreles ruisseaux du Gour et de Pleine-Serve, correspondant exactement au premier axe N.-E, de Mercruy (dont il se détache) au Mont-d'Or ;

$2^{\circ}$ Le coteau de Vaugneray (300-400), compris entre le Chaudanne et l'Iseron ;

$3^{\circ}$ La ride de Messimy, entre le Cnalandresse et le Garon;

$4^{\circ}$ Celle de Soucieu-en-Jarret, entre le Garon et son affluent, le Furon, etc.

Ces dernières rides appartiennent au deuxième axe N.-E., ou chaînon de Francheville.

\section{GHAPITRE II}

\section{CONSTITUTION GÉOLOGIQUE}

Dans la plus grande partie de la région des bas-plateaux lyonnais, le sous-sol est formé presque exclusivement par des gneiss ou des granites, et le sol provient des affleurements de ces roches ou des produits de leur décomposition; il en est ainsi lans toute la partie occidentale, celle qui est la plus rapprochée des monts du 
Lyonnais, et principalement sur les communes de la Tour-de-Salvagny, Lentilly, Marcy, Charbonnieres (en partie), Sainte-Consorce, Pollionnay, Saint-Genis les Ollières (en partie), Grézieux, Vaugneray, Craponne (en partie), Francheville (en partie), Brindas, Chaponost (en partie), Messimy, Soucieu, Brignais (enpartie), Orliénas, Taluyers, Montagny, Chassagny, etc. Mais dans toute la portion orientale, le sol de chacun de nos plateaux est formé, dans une étendue plus ou moins grande, par cles terrains de transport, lehm ou alluvions glaciaires. Le squelette de la région, gneiss et granite, n'apparaît plus généralement que sur les flancs des vallées, ou le long des échancrures qui pénètrent dans l'intérieur des plateaux.

Ajoutons qu'on peut encore observer des terrains de transport :

$1^{\circ}$ Dans le N. de notre région; ce sont alors les alluvions de l'Azergue qui arrivent jusque sur les communes de Lentilly, de la Tour-de-Salvagny et de Dommartin ;

$2^{\text {e }} \mathrm{Au}$ pourtour du Mont-d'Or, entouré d'une véritable ceinture de lehm, que nous étudierons avec cette région naturelle; le lehm se retrouve du reste plus au S. vers la Demi-Lune, Tassin, Fran. cheville (au-dessus des aqueducs de Beaunant) et sur les coteaux du Rhône.

Mais les terrains de transport qui jouent le rôle le plus considérable dans nos bas-plateaux lyonnais sont des alluvions formées principalement par des cailloux roulés cle quartzites alpins, rapportées, par MM. Falsan et Chantre, à l'époque glaciaire qui a - laissé tant de traces dans notre région lyonnaise.

Comme la présence de ces alluvions anciennes ou glaciaires détermine des changements considérables dans la nature du sol et peut influer, par suite, sur sa végétation, il est nécessaire d'entrer' dans quelques détails sur leur extension et de déterminer avec précision leur limite.

D'après nos recherches ${ }^{1}$, ces alluvions recouvrent (roir la carte placée à la fin de l'ouvrage) :

1 On en trouvera la confirmation, pour plusieurs localitès, dans le mémoire de MM. Falsan et Chantre, intitule Catalojue des blocs erratiques, qui paraît, pendant que nous rédigeons ce travail. Voir les Ann. Soc. d'A gric. Lyon, I. X, 1877 (dis. r.bué en 1879), pp. 173 et seq. 
$1^{\circ}$ Le plateau d'Ecully en entier, sauf les bords et les échancrures par lesquelles s'échappent les ruisseaux de la Duchère, de C'haleins ou des Bruyères, d'Écully, etc.;

$2^{\circ}$ La partie méridionale du plateau de Dardilly, sauf son extrémitè même, qui est de nature granitique et ses bords;

$3^{\circ}$ La plaine de la Demi-Lune, de Tassin et la partie du plateau de la Tour de-Salvagny qui lui fait suite, jusqu'au niveau de la limite précédente ;

$4^{\circ}$ Le plateau de l'Aigua et de Champoly jusque vers le bois de l'Étoile ;

$5^{\circ}$ Le tiers oriental du plateau de Méginant;

$6^{\circ} \mathrm{La}$ partie orientale du plateau de Saint-Genis les Ollières jusqu'à une ligne N.-S. passant par le lieu dit les Gouttes et audessous de l'èglise de ce village;

$7^{\circ}$ Le plateau de Craponne, suivant une ligne N.-S., passant au Tourillon et aboutissant à l'O. du Pont-Jambon;

$8^{\circ}$ Sur le plateau de Chaponost, les alluvions s'étendent, dans la partie septentrionale jusqu'au Pont-du-Chêne; de là leur limite extrême se retire vers l'E., passe sous les aqueducs de Chaponost ${ }^{1}$ et s'effile sous l'Orme; elles reparaissent vers la Collonge, d'ou elles s'ètendent jusqu'à Brignais ;

9。 Au-dessous de Brignais, les alluvions se retrouvent sur le bord des plateaux compris entre le Garon, le Chéron et le Caranona; leur limite coupe ensuite les lacets de la route de Lyon a Saint-Étienne au-dessus des Sept-Chemins, sous Taluyers, contourne le mamelon granitique de Montagny et atteint Givors en passant sous le château de Goiffieu.

1 A l'eutrèe même du village de Chaponost se trouve un autre lambeau d'alluvion, mais qui n'a pas climportance au point de vue de la géographie botanique. 


\section{GHAPITRE III}

NATURE DU SOL

La nature du sol des vallées et des bas-plateaux du Lyonnais diffère donc suivant qu'on l'observe dans les parties occidentales ou orientales de cette région.

Les premières possèdent un sol régètal autochtone, c'est-à-dire provenant des èléments mêmes du sous-sol constitué, comme nous l'avons vu, par des gneiss et des granites; dans les secondes, le sol est inclèpendant du sous-sol et formé par des terrains de transport.

Sol autochtone. - Les gneiss et les granites qu'on rencontre dans le Lyonnais présentent de nombreuses différences quant à leur duretẻ et à leur mode de désagrégation; certaines de ces roches, comme les gneiss de l'lle-Barbe, des bords di Garon, des chircuts de Vaugneray et d'Iseron, les granites à petits grains de Francherille, etc., résistent presque entièrement aux actions atmosphériques et restent à l'état de rochers plus ou moins abrupts; d'autres, au contraire, comme les gneiss de Rochecardon, de Chaponost, les granites à grands éléments de la Tour-de-Salvagny, de Vaugneray, de Taluyers, etc., se décomposent rapidement en gore plus ou moins grossier. Mais quelles que soient ces variations, le sol qui provient de la décomposition de ces roches silicéo-alumineuses est toujours un sol sablewx ou argilo-sablenx, que nous appellerous encore, pour employer la terminologie de Thurmann, psam mique ou pélopsammique, suivant la proportion d'alumine que la décomposition des feldspaths et des micas a introduite dans le fond quartzeux du sol même.

Ces roches silicéo-alumineuses, envisagées en grand, constituent 
un sous-sol, non ou peu fissuré et par conséquent imperméable; au contraire, le sol qui provient de leur décomposition est ordinairement perméable à un haut degré, surtout lorsqu'il est exclusivement sableux; mais comme il repose ordinairement sur le sous-sol imperméable, ou que ces parties sableuses plus profondes sont quelquefois intercaléesdans des parties argileuses ou argilo-sableuses, les eaux ne pénètrent pas profondément dans son intérieur et forment ces sources qui surgìssent à chacque pas et après chaque pluie dans les régions granitiques.

Cette disposition du sol et du sous sol explique aussi la fréquence des points marécageux ou légèrement tourbeux qu'on observe en maints endroits, non-seulement dans les prairies des vallées, mais encore sur les plateaux du Lyonnais, dès que l'écoulement de l'eau est empêché par la nature plus argileuse du sol ou parsa disposition en cuvette sur le sous-sol imperméable. Comme exemples de ces points intéressants à cause de leur végétation spéciale, nous citons dès maintenant les marais dits les étangs du Loup (vallée de Beaunant), du Bâtard (plateau de Taluyers), de Lavore (plateau entre Taluyers et Chassagny), et une foule de petits points disséminés dans tout le Lyonnais, principalement dans les environs de Charbonnières, Méginant, Chaponost, etc.

Le sol sableux s'échauffe très vite sous l'influence des rayons solaires, mais se refroidit par contre très rapidement ${ }^{1}$. La conséquence de ce pouvoir absorbant et diffusif est l'accélération de la végétation dans les années sèches et son retard dans les années humides. Mais, en définitive, ces sols paraissent conserver moins de calorique que les sols compactes, comme le prourent et la température moyenne rle leurs sources qui est en général moins élevée que celle des sources des terrains calcaires ${ }^{2}$, et l'ensemble de leur

1 Voy. Lecoe. Études de Géographie botanique, t. I, p. 20.

2 Voy. Thurmans. Phytostatique du Jura, t. I, p. 57, o33.-En attendant que nous donnions la parie de ce lravail consacrée à la clinıatologie lyonnaise, voici quelques faits qui se rattachent à cetle question : $1^{0}$ La température des sources du Lyonnais siliceux ne dépasse pas généralement $11^{\circ} \mathrm{c}$; (Ex. sources de Charbonnières, de Duerne, etc.), tandis que les sources cles coteaux calcaires du Rhòne ont une tempèrature normale de 11 a $13^{\circ}$ c. (S. de Roye, Fontaine, etc.) $2^{\circ}$ Les plantes méridionales 
végétation, moins précoce, formée d'espèces plus septentrionales, plus montagnardes.

La composition chimique de ces sols granitiques et gneissiques est donnée par les analyses suivantes que nous empruntons au mémoire de M. Sauvaneau ${ }^{1}$ :

Granite : sable grossier provenant de la décomposition du granite sur place, aux Landes, commune de Brindas $(300 \mathrm{~m}$.) ; terre de $2^{\mathrm{c}}$ classe, sol moyen, consistance légère, etc. ( $\left.\mathrm{n}^{\circ} 28\right)$.

Résidu après lavage. . . . . . . . . . . 69

Matières insolubles dans acides. . . . . . . 96

Oxyde de fer dissous. . . . . . . . . . . 3,2

Alumine dissoute. . . . . . . . . . . 0,8

Garbonate de chaux. . . . . . . . . "

GNeiss : Gneiss décomposé sur place, au Meillon, commune de Chaponost (325 m.) ; terre de $2^{\circ}$ classe, sol moyen, consistance légère, etc. $\left(n^{0} 29\right)$.

Résidu après lavage. . . . . . . . . . . 73

Matières insolubles dans acides. . . . . . 95

Oxyde de fer dissous. . . . . . . . . . 3,8

Alumine dissoute. . . . . . . . . . . . . 0,6

Carbonate de chaux. . . . . . . . . 0,6

- Gneiss décomposé sur place, mélangé peut-être (?) avec un peu de diluvium, à Chaponost, vers les aqueducs $(310 \mathrm{~m}.) ; 1^{\text {re }} \mathrm{cl}$., sol moyen, consistance légère $\left(\mathrm{n}^{\circ} 30\right)$.

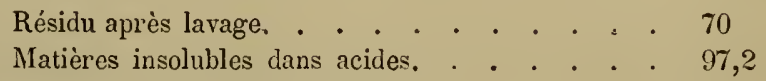

de la Flore lyonnaise sont établies surtout sur les terrains calcaires du Mont-d"Ur et des coteaux du Rhône, bien que la plupart ne soient pas des espèces calcicoles. (Voy, à ce sujet nos études paraissant en ce moment dans le Lyon scientifique. nov. 1879.)

1 Recherches sur la composition des terres régétales (Ann. de la \$oc. d'Agric., hist. nat. et arts utiles de Lyon, t. VIII 18'0̃, p. 419 et seq.) 
Oxyde de fel dissous. . . . . . . . . . 2,2

Alumine dissoute. . . . . . . . . . . . 0,6

Carbonate de chaux. . . . . . . . . . "

- Gneiss décomposé sur place, fragments, à Écully, lieu dit la Charrière-Blanche $(280 \mathrm{~m}.) ; 1^{\text {re }}$ classe, sol profond, consistance moyenne ( $\left.\mathrm{n}^{0} 16\right)$.

Résidu après lavage. . . . . . . . . . 45

Matières insolubles dans acides. . . . . . . . 97,2

Oxyde de fer dissous. . . . . . . . . . . . . 1,6

Alumine dissoute . . . . . . . . . . 1

Carbonatc de chaux. . . . . . . . . . . 0,2

- Gneiss décomposé en sable grossier reposant sur le gneiss en place, au bourg de Dardilly, en face des maisons (320 m.); $2^{c}$ classe, sol moyen rare, consistance légère ( $\left.n^{\circ} 19\right)$.

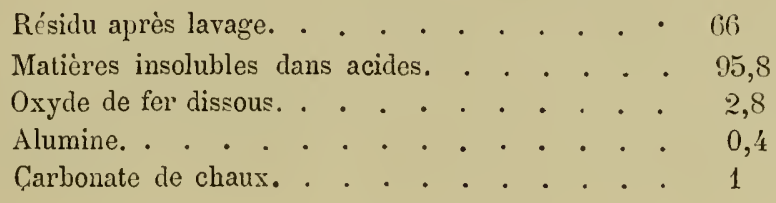

(Le carbonate de chaux doit provenir d'une petite quantité de lehm).

Ces analyses, bien qu'incomplètes et ne donnant pas la richesse en potasse et autres substances importantes pour la végétation, suffisent cependant pour le point de vue spécial auquel nous nous plaçons, la teneur en silice ou en chaux; elles établissent, en effet, que, même dans les terres cultivées, qui ont pu être l'objet d'amendements divers, ce qui domine, c'est la silice, tandis que le carbonate de chaux est nul ou inférieur à 1 pour $0 / 0$.

Sol indépendant. - Le sol végétal formé par les terrains de transport qui recouvrent d'une épaisseur plus ou moins grande les roches silicatées sous-jacentes dans la partie orientale des plateaux du Lyonnais, présente de grandes variations, tant dans ses propriétés physiques que dans sa composition minéralogique. Ces 
alluvion; anciennes sont, en effet, plus ou moins meubles et perméables, suivant que les cailloux roulés de quartzites alpins qui les constituent en grande partie sont, ou non, réunis entre eux par des sables, des argiles ou du carbonate de chaux provenant de la décomposition, soit des fragments de granites, gneiss et d'autres roches feldspathiques, soit des calcaires du Jura, qui accompagnent souvent les quartzites.

Voici quelques exemples que nous avons observés de ces différences :

Au N. de Brignais, à la Collonge, - entre le Pont du Chêne et le Pont-Jambon (commune de Francheville), - vers le Tourillon (commune de Craponne) - sous Dardilly, etc., les alluvions sont constituées par des quartzites libres ou faiblement empâtés dans du sable quartzeux, souvent ferrugineux, devenant quelquefois argileux; c'est un sol végétal très paurre, à Genêts, Bruyères, etc.;

Au bois de l'Étoile (Charbonnières), — sous Dardilly, entre les ruisseaux des Planches et de Serres ${ }_{4}$ et dans beaucoup d'autre; points de la région occupée par les alluvions, mais surtout dans la partie nord, les quartzites sont noyés dans une terre fortement argileuse; sur le plateau de l'Aigua et rers la ferme de Cornatelle, principalement, la prédominance de l'argile donne au sol une compacité et une imperméabilité telles, que les moindres dépressions du sol recueillent les eaux pluviales et donnent asile aux espèces hygrophopiles.

Sur les plateaux de Champoly et de Méginant les quartzites sont fortement réunis entre eux par un ciment argileux et ferrugineux, de manière à constituer des poudingues qui ne sont pas sans analogie d'aspect avec ceux des bords du Rhône, mais qui en diffèrent essentiellement par l'absence de carbonate de chaux.

Voici, du reste, des analyses qui montrent la nature de ces ter rains au point de vue de leur richesse en silice et de leur pauvreti en chaux :

$1^{\circ}$ Terre argileuse à cailloux roulés de quartziles, un peu ferru* 
gineuse; composition moyenne des alluvions de Tassin (route de Sain-Bel, etc.) ${ }^{1}$;

Silice à l'état de sable fin. . . . . . . . . . 875

Silice combinée ou soluble. . . . . . . . . 0,22

Oxyde de calcium. . . . . . . . . . . . 0,64

$2^{\circ}$ Conglomérat ferrugineux à quartzites de Champoly :

Silice à l'état de sable fin. . . . . . . . . 885

Silice combinée ou soluble. . . . . . . . . . 0,34

Oxyde de calcium. . . . . . . . . . . . 0,48

(Ces tableaux ne donnent l'analyse que de la terre qui entoure les cailloux quartzeux, ces derniers constituant souvent la presque totalité du sol.)

En se rapprochant de Lyon et des bords du Rhòne, ces alluvions prennent un caractère différent; en effet, sur le plateau d'Écully, au bord oriental des plateaux de Tassin, de Saint-Genis les Ollières, de Francheville, les alluvions glaciaires deviennent en plusieurs endroits plus ou moins riches en carbonate de chaux, ce qui est dù à la décomposition de cailloux calcaires ou à la présence $\mathrm{du}$ lehm; dans quelques localités, ces alluvions sont manifestement recouvertes par le lehm, qui est quelquefois rempli de concrétions calcaires, de Kupfsteins, comme on peut le voir en montant de Francheville à Chaponost ${ }^{2}$.

On peut même trouver ces alluvions agglomérées en poudingue par un ciment calcaire, par exemple sous Écully, le long de la route de la Demi-Lune, vers les aqueducs de Beaunant, etc.

Mais les sols qui proviennent de ces modifications accidentelles des alluvions sont de très peu deétendue dans la région siliceuse des bas-plateaux lyonnais; on les observe généralement près de sa limite orientale, au voisinage de la région des coteaux du Rhône, avec lesquels il est préfẻrable de les étudier.

1 Toutes les analyses que nous donnons ici sans indication d'origine sont dues ì divers chimistes qui ont bien voulu nous prêter le secours de leurs connaissances spiéciales, entre autres M. Guérin, chef des travaux pharmaceutiques à la Faculté de inédecine, et mou frère, M. Eug. Magnin.

2 Voy. Fournet, Ann. Soc. Agric. de Lyon, t. VII, 1844, p. 380 ; Falsan et Chistre, Catal. des blocs errat., ibid., t. X, 1877, p. 174. 


\title{
GHAPITRE IV
}

\author{
VÉGETATION
}

Pour donner une idée exacte de la végétation des vallées et basplateaux lyonnais, nous allons fournir les documents suivants :

1. Des exemples d'herborisation dans les principales localités de la région;

$2^{\circ}$ Le groupement des espèces rencontrẻes dans ces herborisations, suivant leurs stations habituelles;

$3^{\circ}$ L'énumération systématique des espèces qui constituent la Flore du Lyonnais en distinguant les espèces triviales, communes aux différentes régions secondaires, les espèces plus rares et celles qui sont spéciales aux vallées et aux bas-plateaux;

4. La comparaison de cette végétation avec celle des régions voisines et l'étude de la dispersion géographique des principales espèces, surtout au point de vue des rapports de cette dispersion avec la composition chimique du sol.

\section{\$ 1. - Exemples d'herborisation}

10.
Excursion de Saint-Genis-Laval à la Croix-de-Beaureyard, l'étang du Loup, le vallon de la Font-Marion, Chaponost et le Garon 1.

En descendant de la Croix-de-Beauregard dans la vallée de Beaunant, avant d'arriver à la route de Brignais, on quitte bientôt les terrains de transport pour pénétrer sur le gneiss mis à découvert

1 Voy. pour ces herborisations, les Ann. de la Soc. bot. de Lyon, t. I, p. 120 ; t. II, p. $31,37,80$; - t. III, p. 92,93 ;-t. IV, p. 175, 146;-t. V, p. 112, 178.

Bien que nos comptes rendus d'herborisation different entièrement, ainsi qu'il est facile de s'en assurer, de ceux auxquels nous renvoyous, nous croyons devoir les 
par les phénomènes d'érosion qui ont creusé la vallée de Beaunant, et la partie de celle du Garon qui lui fait suite, au-dessous de Brignais.

Sur les alluvions, on avait noté des plantes triviales, ou indifférentes à la nature $d u$ sol, ou même calcicoles; ainsi par exemple :

Le long des chemins et dans les lieux incultes : Geranium co-lumbinum, Agrostis vulgaris, Alsine tenuifolia, Arenaria serpyllifolia, Trifolium ochroleucum, T. scabrum, Papaver Argemone, P. clubium, Vulpia pseudo-myuros, Myosotis stricta, M. hispida, M. intermedia, Dianthus prolifer, Crassula rubens, Sedum Cepcea, S. reflexum, Bromus madritensis.

Dans les prairies ou les pâturages : Ajuga genevensis. Brunella alba, Lathyrus pratensis, Trifolium Molineri, Koeleria cristata, Arrhenaterum elatius, Avena flavescens, Alopecurus pratensis, etc.

Dans les cultures, les moissons: Sonchus asper, S. oleraceus, Bunias Erucago, Valerianella Auricula, Anthemis Cotula, Agrostis Spica-venti, Lolium temulentum, etc.

Mais dès qu'on atteint la limite des terrains de transport, avec le Sarothamnus scoparius, qui, du reste, se montre déjà sur les alluvions, par une anomalie que nous expliquerons plus tard, on voit apparaître :

Dans les endroits secs, incultes, en outre des espèces citees plus haut :

Vulpia pseudo-myuros.

Nardurus Lachenalii.

Jasione Carioni Bor.
Festuca duriuscula.

Thrincia hirta, etc.

Dans les prairies : Hypochæris raclicata, Roripa pyrenaica, Anthoxanthum odoratum.

citer, comme pouvant servir de preuves à l'appui, dans une certaine mesure.m Mais nous tenons à déclarer que toutes les excursions que nous reproduisons ici ont été refaites par nous plusieurs fois et complétées par de nombreuses courses spéciale. ment entreprises au point de vue de l'ettude des rapports du sol avec la Flore, et en vue de faire la constatation certaine de la nature minéralogique des sols dont on étudiait le tapis végétal. 
Dans les moissons : Linaria striata, Filago montana, et surtout les Rumex Acetosella, Trifolium rubellum, Hypochiris raclicata, Jasione Carioni, nuls ou bien moins abondants dans les champs situés sur les alluvions.

Un petit îlot rocheux de gneiss qui émerge du milieu des terres près du nouveau chemin, sous les Barolles, nous donne une végé tation encore plus spéciale; en effet, au milieu des Erigeron canaclensis, Festuca glanca, Artemisia campestris, Centaurea paniculata, Echium vulgare, Dianthus prolifer, etc., espèces plus ou moins triviales, nous notons:

Erica vulgaris, c. c.

Sarothamnus scoparius, c. c.

Andryala sinuata, $c$.

Hieracium Pilosella, c.
Potentilla argentea, c.

Tormentilla vulgaris, $\mathrm{c}$.

Genista sagittalis.

Nardurus Lachenalii.

et au printemps: Potentilla verna, Pulsatilla rubra.

En atteignant la route de Beaunant à Brignais, nous trouvons un exemple de ces prairies marécageuses situees dans les cuvettes gneissiques si communes dans le Lyonnais; dans celle-ci, connue sous le nom d'Étang du Loup, on peut récolter :

Ranunculus aquatilis.

Helosciadium repens.

H. inundatum.

Roripa amphibia.

Ervum hirsutun.

E. tetraspermum.
Alisma lanceolatum.

Heleocharis palustris.

Glyceria fluitans.

Carex hirta.

C. vesicaria.

C. vulpina.

et moins communes :

Gratiola officinalis.

Galium palustre.

Sagina procumbens.

Enanthe Phellandrium.

E. fistulosa.

Alopecurus fulvus.
Veronica scutellata.

V. scut., var. hirsuta.

Carex paradoxa.

C. paniculata.

Nitella glomerata.

On arrive sur le plateau de Chaponost en suivant une échancrure creusée dans le gneiss : c'est le vallon de la Font-Marion, occupé par une prairie humide, ombragée, à Adoxa Moscha- 
tellina, Luzula campestris, L. vernalis, Stachys silvatica. Valeriana officinalis, Epilobium hirsutum, Scirpus silvaticus, Spergularia mura, Roripa pyrenaica, Carex remota, Gandinia fragilis, Danthonia decumbens.

Sur le plateau de Chaponost, nous nous trouvons en pleine région gneissique : aussi la végétation est-elle tout à fait caractéristique, comme le montrent les séries qui suivent :

Dans les endroits secs, aux bords des chemins, dans les pelouses ou les pâturages, etc. :

Pteris aquilina.

Potentilla verna.

Genista tinctoria.

G. germanica.

Aira caryophyllea.

Trifolium striatum.

T. glomeratum.

Malva moschata.

Pimpinella saxifraga.

Scleranthus annuus.

Vicia latliyroides.
Hieracium Pilosella.

H. Auricula.

Calluna vulgaris.

Rumex Acetosella.

Herniaria hirsuta.

Linaria striata.

Jasione Carioni.

Asperula cynanchica.

Scleranthus perennis.

Herniaria glabra.

Dans les champs, les guérets :

Gypsophila muralis.

Galeopsis ochroleuca.

Filago canescens Jord.

F. lutescens Jord.

F. montana.

Rumex Acetosella.

Spergula arvensis.
Spergula pentandra.

Veronica acinifolia.

Brassica Cheiranthus.

Ervum hirsutum.

Pottia truncata.

Anacalypta lanceolata.

Fissidens adianthoides.

Par suite de l'imperméabilité du sous-sol, les bords des chemins, les fossés et autres dépressions du sol, sont garnis des plantes hygrophiles suivantes:

Juncus glaucus.

J. compressus.

J. bufonius.

Montia minor.

Barbarea præcox.
Roripa pyrenaica.

Mœnchia erecta.

Ranunculus philonotis.

R. aquatilis.

R. peltatus.

Ces mêmes causes determinent, dans toutes les prairies qui 
s'étendent entre Chaponost et le Corandiu, la formation de nombreux points marécageux, qu'on reconnaît de loin aux taches vertnoir formées par diverses espèces de Joncs et habités par :

Cardamine pratensis.

Carex glauca.

Ajuga reptans.

C. stellulata.

Taraxacum officinale.

C. disticha.

T. palustre.

C. panicea.

Alopecurus pratensis.

Mentha Pulegium.

A. utriculatus.

Ophioglossum vulgatum.

En approchant du Corandin, les granites qui percent le sol sont souverts de Racomitrium canescens, Bryum alpinum et pseudotriquetrum; puis on arrive sur les bords de la vallée du Garon, formés par des escarpements de roches silicatées, gneiss et granites, qui s'étendent de là jusqu'à Brignais. En suivant cette direction, on rencontre successivement :

Sur les roches mêmes non désagrégées :

Parmelia consper'sa.

P. perlata.

P. physodes.

P. caperata.

Lecanora Parella.

Lecidea fumosa.

Urceolaria seruposa.

Biatora erythrella.
Parmelia olivacea $f$, prolixa.

P. saxatilis.

Lecidea geographica.

Ramalina pollinaria.

Parmelia triptophylla, nigra.

Umbilicaria pustulata.

Lepraria chlorina.

Dans les fentes des mêmes roches :

Umbilicus pendulinus.

Asplenium septentrionale.

A. Halleri.

Sedum maximum.
Racomitrium canescens.

Grimmia commutata.

G. leucophæa.

G. pulvinata.

Les parties humides supportent Montia minor (plus rarement M.rivularis), Gnaphalium uliginosum, Ranunculus philonotis, Rhynchostegium rusciforme, etc.

Sur le goi'e provenant des granites et des gneiss décomposés:

Polytrichum juniperinum.

P. piliferum.
Astomum crispum.

Bartramia pomiformis. 
Gylindrothecium Montagnei.

Cladonia rangiferina.

Cl. furcata, C. coccifera.

Vicia lathyroides.

Arnoseris minima.

Ornithopus perpusillus.

Teesdalia nudicaulis.

Nardurus Lachenalii.

Polycnemum minus.

Silene Armeria.

Chamagrostis minima.

Veronica verúa.

Asperula cynanchica.
Lycopsis arvensis.

Spergula arvensis.

S. pentandra.

S. Morisonii.

Arenaria leptoclados Guss.

Festuca glauca.

Alsine rubra.

Plantago carinata.

Aira caryophyllea.

A. elegans Gaud.

A. patulipes Jord.

Myosotis versicolor.

Les endroits herbeux, les pelouses sèches sont garnis de :

Veronica acinifolia.

Hypochæris glabra.

Lathyrus sphæricus.

L. angulatus.

Linaria striata.

Festuca glauca.

Anarrhinum bellidifolium.

Jasione montana.

J. Carioni.

Crucianella angustifolia, r.
Peucedanum Oreoselinum.

Andryala sinuata.

Silene nutans.

Phalangium ramosum.

Malva moschata.

Carex præcox.

Trifolinm ochroleucum.

T. subterraneum.

T. Lagopus, $r$.

Thlaspi silvestre, $r$.

Les bords de la vallée, surtout les versants tournés au N., sont garnis de bois constitués par des Chênes, Frênes, Coudriers, Fusains, etc., et qui abritent sous leur ombrage :

Helleborus fœtidus.

Orobus tuberosus.

0 . niger.

Pulmonaria tuberosa.

Saxifraga granulata.

Teucrium Scorodonia.

Phyteuma spicatum.

Veronica Chamæedrys.

V. Teucrium.

Rubus tomentosus.

Melica uniflora.

Viola scotophylla.

Lysimachia nemorum.

Digitalis purpurea.

Centaurea intermedia, $r$.
Carex præcox.

Luzula silvatica.

Aspidium aculeatum.

Polypodium vulgare.

Cystopteris fragilis.

Polystichum Filix-mas.

Brachypodium silvaticum.

Hylocomium triquetrum.

H. squarrosum.

Eurynclium crassinervium.

Pterogonium gracile.

Thuidium delicatulum.

Minium hornum.

Climacium dendroides.

Luzula vernalis. 
Les prairies qui occupent le fond de la vallée renferment, en outre des Poa pratensis, Holcus lanatus, Kceleria cristata, Dactylis glomerata, etc., qui constituent ordinairement ces associations végétales :

Luzula campestris.

L. vernalis.

L. maxima.

Primula officinalis.

P. grandiflora.

Cardamine pratensis.
Viola Reichenbachiana.

V. Riviniana.

Anemone nemorosa.

Corydalis solida.

Adoxa Moschatellina.

Potentilla decipiens.

Dans les parties plus humides :

Roripa pyrenaica.

Cardamine impatiens.

Spiræa Filipendula.
Egopodium Podagraria.

Ranunculus Philonotis.

R. auricomus.

Des mares situées près de Soucieu renferment le Ranunculus hederaceus, et dans le Garon même on peut récolter : Fontinalis antipyretica, Endocarpon fuviatile, divers Spirogyra, les Schizogonium Boryanum, Stigeoclonium protensum, Vaucheria crespitosa, etc.

Excursion à Tassin, le vallon du Ratier, les plateaux de Saint-Genis les ollieres et de l'Aigua'.

De Lyon à Tassin, les propriétés closes et les cultures qui occupent presque toute la surface du sol empêchent les observations de géographie botanique; cependant, dans quelques points encore accessibles, on peut voir que le sol est constitué par les alluvions anciennes ou le lehm; ces terrains sont ordinairement riches en carbonate de chaux; ils constituent quelquefois des poudingues à ciment calcaire, comme on peut le voir au sortir de Vaise, à l'entrée

1 Voy. Ann. Soc. bot. Lyon, t. II. p. 28, : $38 ;-$ t. IV, p. 146; - t. V. p. 112. Et la note p. 40. 
du vallon de Chaleins, sous Écully, et le long de la route de Vaise à la Demi-Lune; vers ce dernier village, on observe de plus une épaisseur considérable de lehm. La végétation de ce sol, analogue à celle que nous avons donnée pour les terrains de transport rencontrés au début de notre première excursion, sera du reste ètudiée dans la partie spécialement consacrée aux Coteaux du Rhône.

En descendant du village de Tassin, dans le vallon du Ratier, on constate d'abord la présence des espèces vulgaires, indifférentes ou rudérales, telles que :

Poa annua.

P. pratensis.

P. compressa.

Eragrostris megastachya.

Agropyrum repens.

Brachypodium silvaticum.

Hordeum murinum.

Barkhausia setosa.

Achillea Millefolium.

Origanum vulgare.

Plantago major.

P. lanceolata.

Veronica agrestis.
Veronica hederæfolia.

Lamium amplexicaule.

L. purpureum.

L. album.

Stellaria apetala.

Lactuca dubia Jord.

Chærophyllum temulum.

Calamintha ascendens Jord.

Chenopodium album.

Ch. opulifolium.

Verbascum Thapsus.

Amarantus retroflexus.

Le sol formé du mélange des alluvions anciennes et du produit de la décomposition des granites donne encore des plantes triviales comme :

Saxifraga tridactylites.

Holosteum umbellatum.

Potentilla verna.

Arabis Thaliana.

Cardamine hirsuta.

Muscari racemosum.

Senecio vulgaris.

Erophila vulgaris.
Capsella Bursa-pastoris.

Cerastium glutinosum.

C. glomeratum.

G. triviale.

Viola odorata.

Stellaria Holostea.

Saxifraga granulata.

ou un peu moins communes comme: Veronica Buxbaumii, Potentilla micrantha, Corydalis solida, Erophila brachycarpa Jord. 


\section{Mais sur les granites on voit aussitôt :}

Sarothamnus scoparius.

Genista pilosa.

Placodium ochroleucum.
Chamagrostis minima.

Candellaria vulgaris.

Notons cependant l'absence du Lecidea geographica.

Dans les prairies qui bordent le Ratier, on trouve, en outre des Graminées habituelles:
Cardamine pratensis.
Primula officinalis.
Ajuga reptans.
P. grandiflora.
Luzula campestris.
P. variabilis.
Viola Riviniana.
Ranunculus acris,
V. Reichenbachiana.
R. bulbosus.
V. hirta.
Isopyrum thalictroides.
V. virescens Jord.
V. scotophylla Jord.
Adoxa Moschatellina.
Anemone nemorosa.
Taraxacum officinale.
Carex digitata.
C. glauca.
C. præcox.
T. levigatum.
Anthoxanthum odoratum.
Equisetum arvense.
Luzula maxima.

Signalons encore Carex polymhiza, assez rare, et dans les parties humides des prairies : Orchis viridis, O. Morio, Enanthe peucedanifolia, Ranunculus auricomus et bien plus rares: Ophioglossum vulgatum, Fritillaria Meleagris.

Dans les bois qui garnissent les versants exposés au N., on remarque :

Fraxinus excelsior.

Cerasus Padus.

Oxalis Acetosella.

Arenaria trinervia.

Adoxa Moschatellina.

Luzula vernalis.

L. campestris.

L. maxima.

Brachypodium silvaticum.

Echium rulgare

Senecio flosculosus.

Carex digitata.

C. montana.

C. remota.
Euphorbia amygdaloides.

Orobus tuberosus.

Pulmonaria angustifolia.

Ribes Uva-crispa.

R. rubrum.

Milium effusum.

Melica nutans.

M. uniflora.

Corynephorus canescens.

Agropyrum caninum.

Avena pratensis.

Poa nemoralis.

Hieracium, $p l . s p$. 
Et les Mousses suivantes:

Mnium rostratum.

Atrichum undulatum.

Isothecium myurum.

Ceratodon purpureus.

Hypnum cupressiforme.

Plagiochila asplenioides.
Leucodon sciuroides.

Anomodon viticulosus.

Brachythecium rutabulun.

Eurynchium prælongum.

Thamnium alopecurum.

Bryum capillare.

Tout le long des vallées du Ratier ou du Charbonnières, sous Tassin, le sol est formé soit par des granites ou des gneiss, soit par des alluvions anciennes à nombreux quartzites alpins noyès dans une terre argileuse jaune ferrugineuse; sur le granite apparaissent le Sarothamne, les Thymus Serpyllum, Linaria striata, etc.; sur les alluvions, le Sarothamne disparaît et il ne reste plus que des plantes indifférentes.

En arrivant sur le plateau de Saint-Genis les Ollières, on se trouve en présence d'une végétation aussi peu caractéristique ; toute la partie orientale du plateau; comprise entre le Ratier et le ruisseau des Ribes est formée par un sol de première qualité, meuble, à cailloux disséminés, et appartenant aux alluvions anciennes; le Sarothamne y est rare, ainsi que les plantes qui l'accompagnent ordinairement; on y rencontre cependant :

Trifolium arvense.

T. rubellum Jord.

Galeopsis dubia.
Brassica cheiranthiflora.

Potentilla decipiens.

Helianthemum guttatum.

mais sur les pelouses sèches, dans les rares endroits non cultivés:

Helianthemum vulgare.

Vincetoxicum officinale.

Medicago falcato-sativa.
M. præcox.

Trigonella monspeliaca.

Antirrlinum Orontium.

c'est-à-dire des espèces ou indiffèrentes ou des sols calcaires.

En continuant plus loin du côté du village de Sant-Genis les Ollières, on arrive à la limite des terrains de transport, limite qui suit à peu près le chemin allant du territoire des Gouttes à Cham. poly ; le granite apparaît alors, et avec lui les Sarothamnes, Hie racium Pilosella, Rumex Acetosella, etc.; ce terrain et cette 
végétation continuent au delà dans toute la partie granitique jusqu'à Vaugneray et les monts du Lyonnais.

Si pour revenir à Tassin, nous suivons le plateau de l'Aigua, situé entre le ruisseau des Ribes et celui de Charbonnières, nous trouvons alors un sol d'alluvions anciennes, fortement argileux, couvert de

Hieracium Pilosella.

H. Auricula.

Arnoseris minima

Holcus mollis.

Hypochæris glabra.

Aira aggr'egata Timeroy.
Filago canescens.

F. lutescens.

F. montana

F. gallica.

F. arrensis, etc.

et dans les parties où l'eau stagne au moins pendant quelque temps: Juncus conglomeratus,.J. bufonius, J. effusus, les Filago cités plus haut, etc.

- Excursion de Lyon à Francheville par le Pont-d'Alä̈. le plateau de Craponne et la vallée de l'Iseron'.

De Lyon au Pont-d'Alaï, on traverse le plateau de Fourvière et de Sainte-Foy, constitué par des granites et des gneiss que recouvrent les alluvions, la boue glaciaire et le lehm; ce platean est donc analogue, prar sa constitution géologique et sa régétation, à celui que nous arons franchi pour aller de Lyon à Saint-Genis et à la vallee de Beaunant.

Déjà, en descendant dans la vallée de l'Iseron par une des nombreuses échancrures qui découpent le bord du plateau on a pu rencontrer les roches silicatées et leur Flore caractéristique; mais ce n'est qu'aprés aroir franchi le Pont-d'Alaï qu'on pénètre dans la région silicense proprement dite du Lyonnais.

On se trouve alor's en présence d'alluvions anciennes à quartzites

1 Voy. Ann. Soc. bot. Lyou, t. II, 1. 23, 70, 98; - t. IV, p. 146 ; - t. V, p. 112 ; et la note page 40 . 
alpins que traversent, de distance en distance, les gneiss formant le sous-sol; le tout donnant naissance à un sol exclusivement siliceux; en effet, sur les gneiss, ce sont :

Lecidea geographica, ccc.

$\mathrm{L}$ fumosa.

Zeora sordida.

Candelaria vulgaris.

Callopisma citrina.

et sur la terre :

Sarothamnus scoparius. c. c.

Hieracium Pilosella.

Potentilla verna.

Jasione Carioni.

Spergula arvensis.
Biatora erythrella.

Grimmia apocarpa.

Racomitrium canescens.

Polytrichum piliferum, etc.

les dernières ubiquistes.

On arrive bientôt sur le plateau de Craponne, compris entre l'Iseron et le Ratier; comme ses voisins, il est formé dans sa partie orientale, par des terrains de transport, occupés par des cultures, à Flore spontanée mixte, mais devenant de plus en plus riche en espèces silicicoles à mesure qu'on approche de la limite des alluvions, laquelle se trouve dans les environs du lieu ditle Tourillon. Ce sont comme exemple: Veronica triphyllos, V. acinifolia, Brassica Cheiranthus, Agrostis Spica-venti, Anthemis arvensis, Ervum monanthos, Holcus mollis, Trifolium rubellum, Galeopsis dubia, Filago sp., etc.

A mesure qu'on approche du Tourillon, c'est-à-dire de la limite des alluvions glaciaires, on roit le Sarothammus scoparius derenir plus abondant, surtout dans les terrains incultes, et aussi fréquent qu'il le sera plus loin sur les gneiss et les granites.

On peut faire la même observation, si du Tourillon on dezcend dans la vallee de l'Iseron, sur le Pont-Jambon, par exemple; on se trouve encore sur les allurions qui forment des mamelons i gauche et à droite du chemin, et dont la limite passe un peu à l'O. du pont; ces allurions sont courertes de Sarothamne, qui manque oll est bien moins abondant sur le gneiss qui affleure sur les bords 
mêmes du chemin avant d'arriver à l'Iseron; il est vrai qu'après avoir franchi le Pont-Jambon, les roches de gneiss sont couvertes de Sarothamne, de Piloselle, de Bruyère et de Jasione.

Si l'on s'arance plus loin sur les coteaux de Francherille et de Chaponost, on suit toujours les allurions, qui deriennent de plus en plus maigres et sont constituées exclusivement par des cailloux de quartzites et des sables siliceux; aussi le terrain est-il ordinaire. ment, dans les lieux incultes, couvert de Bruyères et de Genêt-àBalais.

En revenant à Francheville par le chemin de grande communication $\mathrm{n}^{\circ} 25$, on peut noter, sur ce même plateau, divers faits intéressants au point de vue des rapports du sol avec la végétation ${ }^{1}$; les alluvions sont, en effet, interrompues en plusieurs endroits, soit par des filons de granite, soit par des placages de lehm; sur le gore granitique croissent en grande quantité les Sarothamnes, associés aux autres silicicoles qui les accompagnent habituellement; tandis que ces espèces disparaissent complètement sur le lehm, qui atteint, en quelques endroits, 2 à 3 mètres d'épaisseur et présente de nombreuses concrétions calcaires.

A Francheville-le-Haut, nous retrouvons les escarpements gneissiques des bords de l'Iseron avec les plantes que nous avons dejà récoltées, en partie, sur les mêmes roches des bords du Garon et du Ratier :

Lecidea geographica.

Parmelia conspersa.

P. prolixa.

Ochrolechia Parella.

Busbaumia aphylla.

Grimmia commutata.

Racomitrium canescens.

Les Polythrichs.

Aira flexuosa.
Des Fougères nombreuses.

Potentilla argentea.

Teucrium Scorodonia.

Erophila brachycarpa.

Genista scoparia.

G. sagittalis.

G. germanica.

Luzula maxima.

Enfin, si nous explorons les prairies qui sètendent sur les bords

1 C'est là, non loin de la croisés du chemin allant vers le Pont-Jambon, que se trouve une des rares stations (daus la Flore lyonnaise) de l'Ulez: curopreus, sur des alluvions, en societe arec Sarothamne, Piloselle, etc. 
de l'Iser'on, lorsque la largeur de la vallée le permet, nous notons une Flore identique à celle des autres vallées du Lyonnais que nous avons déjà ètudiẻes; nous nous bornerons à citer, en outre des Primula, Viola, Luzules et autres espèces printanières, ainsi que des Graminées habituelles aux prairies, les :

Scilla bifolia.

Corydalis solicla.

Adoxa Moschatellina.

Isopyrum thalictroides.

Anemone ranunculoides.
Anemone nemorosa.

Spiræa Filipendula.

Potentilla decumbens.

Thamnium alopecurum, etc.

40

Excursion sur le plateau de Champoly, le bois de l'Etoile

le plateau de Méginant,

la vallée de Charbonnières et la Tour-de-Salvagny'.

Nous avons déjà décrit la nature du sol et de la végétation des localités situées entre Lyon et Tassin (voyez précédemment excur$\left.\operatorname{sion} n^{\circ} 2\right)$. Entre Tassin et le plateau de Champoly, on a d'abord les alluvionsqui recouvrent les roches du sous-sol dans toute la plaine de la Demi-Lune et de Tassin; ces alluvions, dans les environs même de Tassin ont peu d'èpaisseur' et sont percées en maints endroits par le gneiss ou le granite; par le fait des cultures, la Flore a peu de caractère et on ne récolte guère le long des chemins que des plantes triviales ou indifférentes, comme les Lamium, les Plantains, les Verbascum, les Chenopodium et les Amarantes, etc. On descend ensuite dans la vallée du Charbonnières, garnie de prairies dont la végétation est tout à fait identique à celle des prairies des bords du Ratier, de l'Iseron et du Garon.

Mais en remontant le plateau, par la route de Sain-Bel, on peut, dès le bas du coteau, noter une végétation bien caractéristique. Si,

I Voy. Ann. Soc. bot. Lyon, t. 1, p. 120, 121; - t. II, p. 28, 41; - t. III, p. 89 ; - t. IV, p. 167; - t. V, p. 11 ; - Bull. Soc. bot. de France, t. XXII, 1876, session de Lyon, p. CLXII. Et notre note p. 40. 
en effet, au lieu de suivre la route on prend le sentier qui la rejoint sur le platean, vers l'auberge de Champoly, on traverse un sol formé par des cailloux roulés, principalement des quartzites, noyés dans une argile jaune, compacte. Or, on y trouve en abondance :

Pinus silvestris.

Quercus sessiliflora.

Castanea vulgaris.

Sarothamnus scoparius.

Festuca beterophylla.

Deschampsia flexuosa.

Senecio nemorosus.
Aira præcox.

A. caryophyllea.

Nardurus Lachenalii.

Vulpia sciuroicles.

Luzula vernalis.

L. Forsteri.

L. multiflora.

Lysimachia Nummularia.

plantes pour la plupart éminemment silicicoles.

De même plus haut, sur les bords de la route, dont les berges sont formées par un conglomérat de quartzites englobés dans une terre ferrugineuse assez compacte, on observe :

Sarothamnus scoparius, c. c.

Erica vulgaris, c. c.

Rumex Acetosella.

Pilosella vulgaris.
Polytrichum commune.

P. piliferum.

Pogonatum aloides.

Bartramia pomiformis.

el de nombreuses espèces du genre Rosa.

Sur tout le plateau, le sol est constitué par des alluvions identi ques, plus ou moins argilo-siliceuses, et partant plus ou moins imperméables.

En effet, le long des chemins, les fossés remplis d'une eau dont la coloration rougeàtre est due à la nature ferrugineuse du sol, sont garnis de Ranunculus aquatilis, Callitriche verna, Juncus conglomeratus, J. effusus, etc., et leurs bords de : Roripa pyrenaica, Peplis portula, etc.

Les terres et les champs sont habités par Aira aggregata, Gypsophila muralis, Scleranthus annus, S. bicnnis, Galeopsis clubia, les Spergules, etc. Lorsque le sol devient très argileux, son imperméabilité permet à certaines espèces hygrophiles de s'y maintenir même pendant les périodes de sécheresse. Un champ argileux, rempli de quartzites roulés, qu'on rencontre avant d'arriver au Bois de l'Étoile, vers la ferme de Cornatelle, supporte une végéta- 
tion bien démonstrative; elle est, en effet, constituée par les espèces suivantes, énumérées en allant des plus communes aux plus rares :

Setaria glauca.

Rumex Acetosella.

Agrostis Spica-venti.

Anthemis arvensis.

Matricaria inodora.

Spergula ar'vensis.

Sp. rubra.

Galeopsis dubia.

Ervum hirsutuul.

Hypochœris radicata.

H. glabra.

Jasione montana.
Holcus mollis.

Aira aggregata.

Juncus bufonius.

Gnaphalium luteo-album.

Epilobium collinum.

Arnoseris minima.

Ornithopus perpusillus.

Hypericum humifusum.

H. Liottardi.

Lotus angustissimus.

Lythrum hyssopifolium.

Trifolium elegans.

Les prairies, qui sont généralement disposées dans les plis de terrain, dans des vallons plus ou moins profonds, renferment, en outre des Graminées triviales :
Scor'zonera humilis.
Orchis maculata.
Cynosurus eristatus.
O. ustulata.
Alopecurus pratensis.
o. Morio.

Lorsque l'écoulement de l'eau devient difficile, on trouve dans les points plus marécageux, avec Scorzonera humilis et les autres espèces énumérées précédemment:
Carum verticillatum.
Orchis laxiflora.
o. latifolia.
Carex flava.
C. pallescens.
Orchis conopea.

0. viridis.

O. palustris.

Pedicularis palustris.

Scutellaria minor.

Cirsium palustre.

Notons aussi l'Ophioglossum vulgatum, qui trouve ici, dans ces prairies à sous-sol imperméable, les mêmes conditions indispensables pour sa végétation que nous avons déjà observées dans ses stations de Chaponost et des autres plateaux lyonnais. 
Des bois couvrent la plupart des pentes, surtout celles exposées au N.; à l'ombre des Chênes, qui en constituent l'essence principale, des Châtaigniers et des Pins, moins communs, mais qui y croissent très vigoureusement, comme dans toutes les régions siliceuses, se développent diverses espèces, entre autres :

Sarothamnus scoparius.

Serratula tinctoria.

Genista tinctoria.

G. sagittalis.

G. germanica.

Erica vulgaris.

Hypericum pulchrum.

H. montanum.

H. tetrapterum.

H. hirsutum.

Peucedanum parisieuse.

Senecio nemorosus.

Luzula maxima.

L. multiflora.

Carex silvatica.

C. hirta.

G. leporina.

C. pallescens.
Millium effusum.

Festuca ovina.

Aira flexuosa.

Danthonia decumbens.

Anthoxanthum odoratum.

Polypodium vulgare.

Leucobr'yum glaucum.

Hypnum purum.

H. cupressiforme.

Hylocomium splendens.

H. triquetrum.

Eurynchium Stockesii.

E. prælongum.

Thuidium filicinum.

Leskea complanata.

Polytrichum piliferum.

P. vulgare.

Les parties liumides, très ombragées, renferment plus spécialement :

Molinia cærulea.

Hyperícum tetrapterum.

Cartum verticillatum.

Sanicula europæa.

Aspidium aculeatum.

A. Filix-fomina.

Polystichum Filix-mas.
Cystopteris fragilis.

Les Luzules

Garex panicea.

Hypnum palustre.

H. cuspidatum.

Jungermannia sp.

Dans le bois de l'Étoile, situé à la linite des alluvions glaciai res, on trouve, en outre des espèces silvatiques énumérées plus haut, les Dianthus cleltoides, Juncus Tenageia, Aira patulipes Jord., Diphyscium foliosum, Webera nutans, Leucobryum glaucum, de nombreux Rosa de la section des Gallicanes, et, accidentellement, le enecio aclonidifolius. 
Un petit vallon sépare le plateau de Champoly et de l'Étoile, de celui de Méginant, situé plus au midi. La végétation est identique ì celle que nous avons indiquée plus haut pour les prairies du plateau de Champoly; on y rencontre les Scorzonera humilis, Carum verticillatum, Orchis ustulata, O. viridis, Ophioglossum vulgatum, etc.

Le plateau de Méginant est recouvert dans tout son tiers oriental par des alluvions glaciaires formées de galets de quartzites solidement reliés par un ciment ferrugineux. Sur le plateau même, occupé en grande partie par les cultures, on observe : Ervum mo. nanthos, Vicia varia, V. lutea, Inula graveolens, Agrostis vulgaris, Muscari comosum, Erythrea Centaurium, Airn caryophyllea, Agrostis canina, Scirpus setaceus, Juncus sp., Scorzonera plantaginea, etc. La présence de quelques-unes de ces espèces est l'indice de l'imperméabilité du sous-sol, qui leur permet de trouver, dans les moindres dépressions du sol, l'humidité suffisante pour leur végétation.

Quelques parties plus accidentées, surtout sur les bords du coteau, supportent des pelouses sèches à Flore éminemment silicicole :

Sarothamnus vulgaris.

Andryala sinuata.

Scleranthus perennis.

Jasione Carioni.

Festuca tenuifolia.
Ranunculus Chærophyllos.

Avena tenuis.

Lithospermum medium.

Mnium hornum.

et de nombreuses Roses.

Du reste, en se rapprochant de la limite des alluvions, on voit la Flore, tout en restant silicicole, se modifier légèrement; nous avions déjà observé ce fait en allant du Pont-d'Alaï à Vaugneray ; on peut le vérifier ici en allant soit de Méginant, soit du Bois-del'Étoile, à Marcy-les-Roses; on voit alors les Teesclalia mudicaulis, Ornithopus perpusillus, Myosotis versicolor, Narlus stricta, Veronica verna, Bryum alpinum, etc., rares ou nuls sur les alluvions alumino-siliceuses, devenir de plus en plus fréquents sur le gore granitique. 


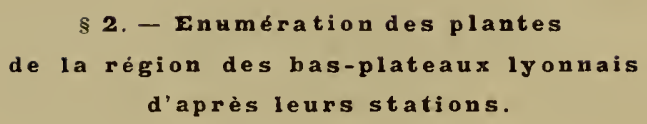

Bien que l'examen des divers comptes rendus d'herborisations que nous venons de donner démontre que la régétation de la région des Bas-Plateaux possède un caractère général, dèterminé par la présence constante des Sarothamnes, Jasiones, Scléranthes, Racomitrium, Polytrichs, etc., cependant, comme on a pu le voir par la même étude, cette végétation revêt des nuances diverses suivant les changements qui surviemnent dans l'état physique clu sol, dans la disposition topographique des lieux, leur orientation, etc. Certaines espèces, en effet, croissent de préférence dans les endroits incultes, sur les rochers, dans les sables ou parmi les bruyères; d'autres ne se plaisent que dans les endroits couverts, frais, à l'ombre des bois, ou dans les prairies, ou bien dans les cultures; enfin, les eaux donnent asile à des plantes qui ne se rencontrent jamaiș dans un autre milieu.

Nous allons donc énumérer successivement les espèces des habitats suivants :

$1^{\circ}$ Rochers nus, secs ou mouillés, formant des éboulis ou décomposés en gores, recourerts ou non de pelouses ou de Bruyères;

$2^{\circ}$ Bois, en distinguant les bois secs des plateaux et ceux plus frais des vallées;

$3^{\circ}$ Prairies, depuis les pàturages secs jusqu'aux prés marécageux;

$4^{\circ}$ Lieux argileux humides, mares, ruisseaux et rivières;

Јo Cultures, etc.

Il nous restera ensuite à examiner si la Flore des alluvions glaciaires siliceuses présente quelque diffẻrence arec celle de la région granitique proprement dite, et nous indiquerons à l'appui de cette comparaison les espèces qui croissent dans les lieux incultes, les bois et les cultures de ces terrains de transport. 
$1^{\circ}$ Rochers (Granites et gneiss formant les versants des vallées du Ratier, de l'Iseron (jusqu'à Francheville), du Garon (jusqu'à Brignais); - des escarpements sur les bords des ruisseaux de Rochecardon, de Chaleins, d'Écully, des Planches, de Charbonnières, apparaissant à travers le lehm ou les alluvions glaciaires ; - des affleurements à la base du massif du Mont-d'Or, des plateaux de Fourvière, d'Oullins, du Perron, de Saint-Genis-Laval, d'Irigny et Millery, ou dans les échancrures creusées à leur pourtour).

Les parties nues, exposées au soleil, à surface non décomposée, ne permettent la végétation que de quelques cryptogames, telles que :
Lecidea geographica 1.
Parmelia conspersa.
L. fumosa.
Biatora ferruginea.
Callopisma citrina.
C. aurantiaca.
Placodium saxicolum.
P. olivacea $f$. prolixa.
Umbiliearia pustulata.
Grimmia pulvinata.
Racomitrium canescens.
Homalothecium sericeum.

Dans les fentes des rochers, surtout lorsqu'ils commencent à se décomposer, on observe : Festuca duriuscula, F. glauca, $S a-$ rothammus vulgaris, Spergula Morisonii (Garon), Deschampsia flexuosa, Sedum maximum; - Epilobium lanceolatum, Bupleurum Jacquinianum, B. junceum, Epilobium collinum, Sedum elegans (Charbonnières), Silene Armeria; ces dernières, rares, habitent principalement la vallée du Garon.

Lorsqu les rochers sont garantis des rayons du soleil ou tournés vers le N., comme cela arrive au fond des vallées de Char-

1. Sauf indication contraire, toutes les fois que dans une énumération les espèces ne sont pas indiquées dans l'ord̉re systématique, c'est qu'elles sont disposées en allant des plus communes (surtout en individus) aux plus rares. 
honnières, hu Ratier, de l'Iseron et du Garon, ils se recourrent alors de Racomitrium canescens, Girmmia leucophra, G.commutata, Bartramia pomiformis, Hedrigia ciliata, Cylindrothecium Montagnei, Ramalina pollinaria, et plus rarement le Bartramia ithyphylla; dans leurs fissures s'établissent de nombreuses Fougères, entre autres:

Asplenium septentrionale.

A. Halleri.

A. Breynii.
Cystopteris fragilis.

Umbilicus pendulinus.

Quelquefois ces rochers sont parcourus par des filets d'eau qui permettent à des plantes hygrophiles, surtout à des Mousses et des Algues, de se développer : Montia rivularis, Gnaphalium uliginosum, Rhynchostegium rusciforme, Philonotis fontana, Vaucheria caspitosa, Conferva bombycina, etc.

Observation. - Le Lecidea geographica, dont la présence caractérise les régions siliceuses, même pour les phytostaticiens partisans de la prépondérance de l'influence physique ${ }^{1}$, ne se rencontre pas uniformèment dans toute l'étendue du Lyonnais granitique; nous avons constaté qu'il est commun surtout dans les vallées du Garon et de l'Iseron; il devient plus rare, à partir de Tassin, dans les vallèes du Ratier et du Charbonnières et manque complètement dans celles qui entourent la base du Mont-d'Or, bien que les roches gneissiques et granitiques $y$ affleurent dans toute leur étendue ${ }^{2}$.

Les Umbilicaria pustulata, Grimmia leucophcea, G. com mutata semblent aussi localisés dans les vallées du Garon et de l'Iseron, surtout dans la première, où ils sont assez répandus; ils paraissent être descendus des monts du Lyonnais.

\section{$2 \circ$ Gneiss et granites décomposés (mêmes localités).}

Les granites et certains gneiss donnent en se décomposant un

1 Voy. Thurmann, Phytost. du Jura, t. I, p. 421, 424 et 425.

2 Nous l'avons cependant rencontré dans le massif du Mont-d Or, au fond du vallon de Saint-Romain de Couzon, mais sur les calcaires siliceux du Civet. 
sol sableux, connu sous le nom de gore; parmi les nombreuses espèces psammophiles qui y croissent, nous citerons :

D'abord les plantes triviales, comme : Erigeron canadensis, Artemisia campestris, Potentilla verna, Festuca lenuifolia, Serpyllum vulgare, Teucrium Chamxdrys, Gypsophila saxifraga, Eryngium campestre, Carlina vulgaris, Echium vulgare, Dianthus prolifer, Genista sagittalis, etc.

Les espèces plus spéciales :

Sarothammus scoparius.

Pilosella vulgaris.

Rumex Acetosella.

Trifolium rubellum Jord.

Racomitrium canescens.

Polytrichum piliferum.

Jasione montana.

Scleranthus perennis.

Ornithopus perpusillus.

Cerastium brachypetalum.

Linaria striata.

Spergula pentandra.

Nardurus Lachenalii.

Nardus stricta.

Arnoseris minima.

Les Sagines.

Myosotis versicolor.

\section{Et plus rares :}

Crucianella angustifolia.

Echinospermum Lappula.

Silene Armeria.
Vicia lathyroides.

Tormentilla officinalis.

Anarrhinum bellidifolium.

Mibora verna.

Sedum reflexun.

Teesdalia nudicaulis.

T. Lepidium.

Hieracium Auricula.

Andryala sinuata.

Vulpia pseudo-myuros.

Spergula Morisonii.

Phleum nodosum.

Carex Schreberi.

Aira canescens.

Herniaria glabra.

Plantago carinata.

Armeria plantaginea.
Myosotis Balbisiana.

Aira elegans.

Ulex europæus.

Sur les plateaux, le gore provenant de la décomposition des affleurements de granites qui percent le sol, repose quelquefois sur des couches imperméables ou dans des sortes de cuvettes qui retiennent les eaux pluviales ou de sources; on a alors des sables humides à :

Montia minor.

Roripa pyrenaica.
Sagina procumbens.

Hypericum humifusum. 
Pedicularis silvatica.

Bryum alpinum.

Sagina erecta.

B. pseudotriquetrum.

Les gneiss forment d'autres fois, en se délitant, des éboulis de fragments plus ou moins volumineux, dont la végétation diffère quelque peu de celle du gore. A mesure que les débris pierreux deviennent plus considérables, on voit disparaitre les Teesdalia nudicaulis, Ornithopus perpusillus, Arnoseris minima, Anarrhinum, etc., et les espèces suivantes les remplacer ou devenir prédominantes :

Hellebor'us fotidus.

Potentilla rupestris.

Crassula rubens.

Sedum reflexum.

Andryala sinuata.
Scrofularia canina.

Linaria striata,

Phalangium ramosum.

Phleum nodosum.

Nardurus Lachenalii, etc.

$3^{\circ}$ Pelouses : Les bords des plateaux, les lisières et les clairières des bois, constituent des pelouses sèches formées par :

Festuca tenuifolia.

F. glauca.

Linaria striata.

Dianthus Carthusianorum.

Silene nutans.

Thymus Chamædrys.

Rumex Acetosella.

Sarothamnus scoparius.

Alyssum calycinum.

Helianthemum vulgare.

Dianthus Armeria.

Cerastium obscurum.

Linum catharticum.

Vicia lathyroides.

Luzula Forsteri.

L. campestris.

Anthoxanthum odoratum.

Aira caryophyllea.

Vulpia pseudo-myuros.
Festuca rubra.

Nardurus Lachenalii.

Anarrhinum bellidifolium.

Potentilla argentea.

Pulsatilla rubra.

Genista anglica.

G. sagittalis.

Trifolium striatum.

T. glomeratum.

Jasione Carioni.

Thrincia hirta.

Erica vulgaris.

Poterium dictyocarpum.

Trifolium scabrum.

Vicia angustifolia.

Hieracium Auricula.

Veronica verna.

A vena tenuis.

Trifolium subterraneum.

et les Polytrichum juniperinum, $P$. piliferum, Pogonatum nanum, $P$. aloides, Bryum atro-purpureum, etc. 
Plus rarement Ranunculus Chcerophyllos, Trigonella monspeliaca, Aira aggregata Tim.; les Potentilla decumbens, Trifolium Lagopus, Ranunculus cyclophyllus Jord. (forme très remarquable du $R$. monspeliacus), sur les coteaux du Garon; les Polygala depressa, Bupleurum tenuissimum, à Charbonnières, etc.

$4^{\circ}$ Bruyères. - Elles occupent ordinairement les affleurements des granites à la surface des plateaux, en les délimitant quelque fois fort exactement, comme on peut le vérifier dans les environs de Charbonnières, de Soucieu, de Taluyers, etc; la végétation de ces garennes, varennes, bruyères, a beaucoup de rapport arec celle des pelouses sèches; elle est composée des mêmes éléments, mais groupés d'une façon différente, ainsi que le montre l'association qui suit :

Erica vulgaris.

Pteris aquilina.

Sarothamnus vulgaris.

Teesdalia nudicaulis.

Anarrhinum bellidifolium.

Ornithopus perpusillus.

Spergula pentandra.

Potentilla Tormentilla.

Euphrasia officinalis.

Veronica ver'na.

Agrostis vulgaris

Thrincia hirta.

Jasione Carioni.
Festuca duriuscula.

Hieracium Pilosella.

H. umbellatum.

Potentilla ver'na.

Polygala vulgaris.

Rumex Acetosella.

Viola cauina, etc.

Polytrichum piliferum.

Racomitrium canescens.

Cladonia rangiferina.

Cl. furcata, etc.

Ulex europæus (rare).

$5^{\circ}$ Bois. - Ce qui caractérise la végétation des Bois du Lyonnais, c'est la belle venue des Chênes, du P'in silvestre et du Châtaignier

Les arbres ou arbrisseaux qu'on y rencontre ensuite le plus souvent, sont le Charme, l'Orme, le Coudrier, l'Érable champêtre, le Fusain, le Nerprun, l'Aubépine, etc.

La végétation qui se développe sous leur ombrage diffère suivant que les Bois occupent les terrains secs des plateaux ou les versants frais, exposès au N., des vallées.

a. Bois des Plateaux (la Tour-de-Salvagny, Marcy, Charbonnières, Méginant, Francheville-le-Haut, etc.). 
Les Chênes, Châtaigniers, Charmes, etc., abritent les espèces communes suivantes :

Luzula vernalis.

L. Forsteri.

L. multiflora.

Primula grandiflora.

Anemone nemorosa.

Viola scotophylla Jord.

V. Reichenbachiana.

Orobus tuberosus.

Pulmonaria tuberosa.

Brachypodium silvaticum.

B. pinnatum.

Corydalis solida.

Sarothamnus scoparius.

Genista sagittalis.

G. tinctoria.

Scabiosa Succisa.

Stellaria graminea.

Dianthus Carthusianorum.

Hypochæris maculata.

Veronica officinalis.

Melittis Melissophyllum.

Convallaria Polygonatum.

C. multiflorum.
Andryala sinuata.

Hieracium umbellatum.

Teucrium Scorodonia.

Anthoxanthum odoratım.

Aira flexuosa.

Danthonia decumbens.

Festuca ovina.

Hypericum montanum.

H. pulchrum.

Campanula Rapuncults.

Serratula tinctoria.

Centaurea nemoralis Jord.

Atrichum undulatum.

Polytrichum commune.

P. piliferum.

Pogonatum aloides.

P. nanum.

Dicranum scoparium.

D. heteromallum.

Trichostomum pallidum.

Astomum crispum.

Ceratodon purpureus.

Les Diphyscium foliosum, Buxbaumia aphylla plus rares; les Hypnum Schreberi, Webera nutans, Leucobryum glaucum, à Charbonnières.

Quelques espèces phanérogames ontaussi des stations spéciales: Dianthus deltoides, très commun à Tassin, Charbonnières ; Quer.cusapennina, bois taillis de Charbonnières; Aira patulipes Jord., Campanula Cervicaria, au bois de l'Étoile et au Garon; Gnaphalium silvaticum, à Tassin; Centaurea intermedia Cariot, forme du C. lugclunensis Jord. (var. du C. montana), trouvé audessus du moulin du Barail ; le Senecio adonidlifolius, dont quelques pieds ont été trouvés à Tassin et récemment à Charbonnières.

b. Bois frais des versants exposés au $N$. (Vallées du Charbonnières, du Méginant, du Ratier, de l'Iseron, du Garon et de ses affluents ; vallons de Rochecardon, Chaleins, Dardilly, etc.) 
Ces Bois sont composés des arbres et arbrisseaux des Bois des plateaux, et en outre des Fraxinus excelsior, Populus nigra, Rhammus Frangula, Cerasus Padus, des Saules ( $S$. cinerea, etc.).

A l'ombre de ces bois croissent :

Anemone nemorosa.

Viola Riviniana.

Pulmonaria tuberosa.

Primula officinalis.

Lysimachia Nummularia.

Carex silvatica.

C. remota.

Festuca heterophylla.
Luzula silvatica.

Phyteuma spicatum.

Saponaria officinalis.

Aquilegia vulgaris.

Hellebor'us foetidus.

Ranunculus nemorosus.

Lactuca muralis.

\section{Dans les endroits plus humides :}

Epilobium hirsutum.

E. parviflorum.

Circæa lutetiana.

Peucedanum parisiense.

Egopodium Podagraria.

Valeriana officinalis.

Mohringia trinervia.

Asperula odorata.

Hypericum tetrapterun.

H. pulchrum.
Geranium pyrenaicum.

Oxalis Acetosella.

Potentilla micrantha.

Lysimachia nemorum.

Myosotis silvatica.

Euphorbia dulcis.

Aspidium aculeatum.

Polystichum Filix-mas.

P. spinulosum.

Athyrium Filix-fœemina.

\section{Des Mousses nombreuses les habitent :}

\section{Hypnum purtm.}

H. cupressiforme.

H. molluscum.

Hylocomium triquetrum.

H. splendeus.

Thuidium delicatulum.

Th. tamariscinum.

Eurynchium longirostre.

E. prælongum.

E. crassinervium.

Brachythecium populeum.

$\mathrm{Br}$. rutabulum.
Brachy thecium rivulare.

Amblystegium serpens.

Isothecium myurum.

Mnium punctatum.

M. cuspidatum.

M. hornum.

M. affine.

M. rostratum.

M. undulatum.

Fissidens bryoides et adianthoides.

Climacium dendroides.

Notons spécialement le Thaminium alopecurum, dans la vallèe 
de l'Iseron; et les Eurynchium Stockesii, Pterogonium gracile, Plagiothecium denticulatum, dans le vallon de Charbonnières.

Les rares phanérogames suivantes se trouvent dans presque toutes les vallées ombragées et fraîches énumérées plus haut, soit dans les bois, soit à leur limite, dans les prairies humides : Sanicula europrea, Adoxa Moschatellina, Cardamine impatiens, Isopyrum thalictroides, Anemone ranunculoides.

Le Chrophyllum aureum, dans les mêmes stations à Beaunant, Francheville, Tassin ; le Senecio silvaticus, à Charbonnières etVaugneray; les Carex pilulifera, C. polyrhiza à Charbonnières et Dardilly; le Viscaria purpurea, à Charbonnières; le Maianthemum bifolium à Tassin; le Paris quadrifolia, dans les vallons de Saint-Didier au Mont-d'Or et Dardilly; le Veronica montana au N. de Chasselay, à -Rochecardon et au Pont-d'Alaï ; les Hesperis matronalis, Thaspi silvestre, sur les bords du Garon ; le Digitalis purpurea, qui descend des monts du Lyonnais jusqu'à Vaugneray et suit les bois de la vallée du Garon jusqu'audessus du moulin du Barail.

$6^{\circ}$ Les Prairies occupent ordinairement le fond des vallées, ains que nous l'avons explique plus haut (Voyez Topographie, p. 30); mais on en rencontre aussi dans des dépressions à la surface des Bas-Plateaux.

Les végétaux qui les composent sont les Graminées habituelles:

Poa pratensis,

Dactylis glomerata.

Cynosurus cristatus.

Anthoxanthum odoratum.
Alopecurus pratensis.

Phleum pratense.

Deschampsia cospitosa, etr.

De plus, on y voit successivement fleurir les:

Primula officinalis, variabilis et grandiflora.

Viola Reicheubachiana, odor'ata, Anemone nemorosa.

Orchis ustulata.

O. Simia.
Lathyrus pratensis. Hypochæris radicata. Bunium verticillatum. Scorzonera plantaginea Leontodon autumnalis. Nardus stricta.

ces dernières caractéristiques. 
Dès que ces prairies deviennent plus humides, on roit les espèces qui suivent s'ajouter aux précédentes :

Cardamine pratensis.

Silaus pratensis.

Heracleum Sphondylium.

Lychnis Flos-Cuculi.

Viola Riviniana.

Sanguisorba officinalis.

Roripa pyrenaica.

Vicia Cracea.

Rhinanthus glabra.

Spiræa Filipendula.

Festuca cærulea.

Alopecurus utriculatus.
Barbarea vulgaris.

B. præcox.

Rannnculus auricomus.

Lotus uliginosus.

Orchis viridis.

O. laxiflora.

Inula Pulicaria.

Symphytum officinale.

Carex hirta.

G. distans.

C. panicea.

Taraxacum palustre, etc.

Notons spécialement : Senecio erraticus et aquaticus, à Dar dilly; Enanthe pimpinelloides, sous Mornant; Crepis paludosa, aux bords du Garon; Symphytum tuberosum, à Franche-ville, Brignais; Fritillaria Meleagris, sur les bords du Ratier, sous Tassin; Ophioglossum vulgatum, dans beaucoup de prairies à soussol imperméable formant la transition avec la catégorie suivantes.

Prairies marécageuses. - Dès que le sous-sol imperméable ou le défaut de pente ne permet plus l'écoulement des eaux, il se forme dans les prairies des parties marécageuses, quelquefois même de véritables petites tourbières, où l'on voit apparaître une Flore spéciale dont voici les principaux représentants :

Ranunculus Flammula.

Roripa amphibia.

Lychnis Flos-Cuculi.

Stellaria uliginosa.

Enanthe fistulosa.

E. Phellandrium.

(E. peucedanifolia.

Galium palustre.

Cirsium palustre.

Gratiola officinalis.

Veronica scutellata.

Pedicular'is palustris.

Scutellaria galericulata.

Sc. minor.
Myosotis palustris.

Pedicularis palustris.

Mentha Pulegium.

Salix cinerea.

Orchis latifolia.

0 . viridis.

Eriophorum latifolium.

E. intermedium, etc.

Alopecurus fulvus.

A. geniculatus.

Hypnum purum.

H. cuspidatum.

Bracliy thecium rutabulum.

B. rivulare. 
Les Juncus conglomeratus, diffusus, etc., C'arex flava, leporina, etc., et d'autres espèces plus rares, comme Carex paradoxa, C. paniculata, Scirpus supinus, Sc. setaceus, etc.

Ces marais, plus ou moins tourbeux, sont établis soit le long des rivières, principalement du Charbonnières, du Mèginant, du Ratier, du Garon, soit dans des cuvettes gneissiques ou granitiques, comme les étangs du Loup (vallée de Beaunant), du Bâtard (sous Taluyers), de Lavaure (à l'O. de Souzy et de Chassagny), et les nombreux points de peu d'étendue disséminés sur tous les BasPlateaux dans les environs de Méginant, Chaponost, Saint-GenisLaval, etc. Nous renvoyons du reste leur étude au moment où nous exposerons la Flore des tourbières et des marais tourbeux.

\section{Flore aquatique. - a. Lieux argileux humides :}

Roripa pyrenaica.

Lotus uliginosus.

Ranunculus philonotis.

Nasturtium silvestre.

Hypericum humifusum.

Montia minor.

Corrrigiola littoralis.

ces deux derniers rares.

b. Bord cles fossés :

Barbarea vulgaris.

Roripa pyrenaica.

Inula dysenterica.

Alopecurus geniculatus.

Scrofularia nodosa.
Peplis Portula.

Holosteum umbellatum.

Gnaphalium luteo-album.

Gn. uliginosum.

Inula dysenterica, etc. Myosurus minimus.

Juncus capitatus.

\section{c. Mares, ruisseaux, rivières :}

Ranunculus aquatilis.

R. confusus.

R. fluitans.

R. hederaceus (Soucieu, le Garon).

R. peltatus (Chaponost).

Montia rivularis.

M yrioph yllum verticillatum.
Myriophyllum alternifolium.

Callitriche, sp.

Potamageton, sp., etc.

Helosciadium, sp., etc.

Hypnum fluitans.

Amblystegium irriguum.

A. riparium. 
Philonotis fontana.

Rhynchostegium r'usciforme.

Fontinalis antipyretica.
Eudocarpon fluviatile.

Lemanea fluviatilis.

Vaucheria cæspitosa, etc.

Nous bornons là nos indications sur la Flore aquatique, les données qu'elle fournit offrant peu d'intérêt pour le point de vue particulier auquel nous nous plaçons en ce moment dans l'étude de la végétation du Lyonnais; nous signalerons seulement, avec $\mathrm{M}$. le docteur Saint-Lager, l'absence singulière dans tous nos cours d'eau des Cinclidotus fontinaloides, riparius et aquaticus, si communs dans les ruisseaux et les rivières des régions calcaires, du Bugey, par exemple!

8' Cultures. - Dans les champs ordinairement sablonneux des plateaux du Lyonnais granitique, on remarque :

$\begin{array}{ll}\text { Brassica Cheiranthus. } & \text { Filago arvensis. } \\ \text { Burias Erucago. } & \text { F. gallica. } \\ \text { Viola tricolor. } & \text { Linaria simplex. } \\ \text { Gypsophila muralis. } & \text { L. arvensis. } \\ \text { Spergula arvensis. } & \text { L. Pelliceriana. } \\ \text { Alsine segetalis. } & \text { Rhinanthus hirsuta. } \\ \text { A. rubra. } & \text { Veronica triphyllos. } \\ \text { Trifolium arvense. } & \text { Galeopsis ochroleuca. } \\ \text { Vicia monanthos. } & \text { Teucrium Botrys. } \\ \text { Aphanes arvensis. } & \text { Mibora verna. } \\ \text { Filago germanica. } & \text { Agrostis Spica-venti. } \\ \text { F. montana. } & \text { A. alba. }\end{array}$

Plus rares: Saponaria Vaccaria, Lithospermum permixtum. En approchant des coteaux du Lihone on trouve plus spécialement : Ranunculus monspeliacus, Papaver dubium, P. Argemone, Reseda Phyleuma, Vicia varia, Lathymus Nissolin, Matricaria Chamonilla, Ornithogalum umbellatum, etc.

Haies. - Elles sont composées des arbrisseaux habituels: Cratiegus oxyacantha, Pmuns spinosa, A eer campestre, Evonymus europieus, Viburnum Lantana, Ligustrum rulgare, Cory-

1 Voy. Aun. Soe. but. Lyon, t. 11, p. 30. 
lus Avellana, Ulmus campestris, etc. Elles se distinguent, dans le Lyonnais siliceux, par l'absence ou la rareté du Berberis vulgaris et du Cerasus Mahalel, et la fréquence des Prunus insititia, $P$. fruticans, Mespilus germanicà, Ilex aquifolium, et surtout des nombreuses espèces du genre Rosa ${ }^{1}$. On y rencontre quelquefois des pieds d'Ulex europaus.

B. SOUs-RĖGion des AlLuvions alpines Siliceuses

Leur Flore présente la plus grande analogie arec celle de la sous-région granitique, comme le montrent les énumérations que nous allons faire des principales plantes habitant les lieux incultes, les bois et les cultures, seules stations intéressantes de cette région.

1. Lieux incultes, pelouses, etc. :

Sarothamuus vulgaris.

Genista germanica.

G. anglica.

Luzula vernalis.

L. Forsteri.

Polytrichum commune.

P. piliferum.

l'ogonatum aloides.

Bartramia pomiformis.

Erica vulgaris.

Rumex Acetosella.

Andryala sinuata.
Festuca tenuifolia.

Jasione Carioni.

Avena tenuis.

Helianthemum vulgare.

Vulpia sciuroides.

Aira caryophyllea.

A. precox.

Nardurus Lachenalii.

Seleranthus perennis.

Potentilla collina.

Anemone rubra.

Ranuneulus Chærophyllos.

2. Bois (Dardilly, Charbonnières, Champoly, Méginant, plateau de l'Aigua à Tassin, etc.) :

Quer'cus pedunculata.

Q. sessiliflora.

Q. apennina.

Castanca vulgaris.

Pinus silvestris, etc.
Anemone nemorosa.

Viola scotophylla.

Hypericum pulchrum.

Andryala sinuata.

Aira patulipes.

1 Voy. Bocluv. Enumérat. des Rosiers de la Flure lyonmaise (Bull. Soc. bot. de F'rance, t. XXIII, 1876, session de Lyon, p. XLVIl, xLTHI). 
Festuca heterophylla.

Deschampsia flexuosa.

senecio nemorosus.
Centaurea nemoralis.

Scr'ratula tinctoria.

Soliclago Virga-aurea, etc.

et la même série de Mousses que pour les bois des plateaux granitiques.

$3^{\circ}$ Cultures : ce sont elles qui présentent les plus grandes différences par leur sol argileux et argilo-siliceux et leur végétation composée de :

Ranunculus parviflorus.

Spergula arvensis.

Sp. rubra.

Alsine scgetalis.

Gypsophila muralis.

Holosteum umbellatum.

IJypricum humifusum.

Lotus tenuis.

L. diffusus.

Lythı'um Hyssopifolia.

Filago montana, gallica, arvensis, germanica.

Inula graveolens.

Hypochæris glabra, radicata.

Linaria Pelliceriana.
Veronica acinifolia.

Ervum hirsutum.

E. monanthos.

Vicia raria.

V. lutea.

Anthemis arvensis.

Matricaria inodora.

Juncus bufonius.

J. hybridus.

J. Tenageia.

Holcus mollis.

Agrostis alba.

A. vulgaris.

A. Spica-venti.

Et les Rumex Acetosella, Scleranthus ammus, Sc. biennis, Ornithopus perpusillus, Arnoseris minima, Trifolium rubellum, Jasione montana, moins fréquents que dans la région granitique.

\section{Pottia truncata.}

Anacalypta lanceolata.

Pleuridium subulatum.

Phascum cuspidatum.
Physcomitrium piriforme.

Enthostodon fasciculare.

Brachythecium rutabulum.

B. glareosum, etc.

Et plus rares: Myosumus minimus, Centunculus minimus, Cicendia filifomis.

Les alluvions siliceuses, tout en ayant une Flore identique dans son ensemble avec celle de la sous-région granitique proprement dite, présentent cependant quelques parlicularités qui méritent d'ètre notées: 
1. Tandis que les Sarothamnus vulgaris, Genista anglica, Pteris aquilina sont aussi fréquents dans une région que dans l'autre, les Teestlalia nutlicaulis, Scleranthus perennis, Amose'is minima, Naidurus Lachenalii, si abondants dans les terrains sableux provenant de la décomposition des granites, semblent presque nuls sur les allurions, ou tout an moins bien moins fréquents ${ }^{1}$.

2. Les alluvions glaciaires semblent avoir aussi le privilège de posséder plus particulièrement certaines espèces, comme les Roses de la section Gallicanie, le Leucobryum glaucum, qui existe abondamment en différents points du Bois-de-l'Étoile (Charbonnières) sur les alluvions, et que je viens de retrouver aussi abondant, sur le mème terrain, à l'extrémité méridionale du plateau de Dardilly².

3. Les terres argileuses dominent sur les plateaux recouverts par les alluvions glaciaires, surtout sur ceux de Champoly, Méginant et de l'Aigua; elles y déterminent, dans les cultures, par exemple, une végétation un peu différente de celle des moissons du Lyonnais granitique, et qui offre beaucoup d'analogie avec celle de la Dombes; mais un certain nombre d'espèces sont communes aux denx sous-régions, et leur présence sur des sols si différents au point de vue pliysique, possédant d'autre part ce caractère commun d'être dépourvus de chaux, est peut-ètre une des preuves les plus frappantes de la prédominance de l'influence chimique dans la dispersion de ces végétaux.

1 Comparez un fait exactement semblable signalé pour les mêmes plantes (mais sur. d'aulres terrains) jar M. B. Martin, dans les environs de Campestre. (Bull. Soc. Lot. France, 1875, t. XXI, session d'Angers, p. Xxxvir.) Du reste, des recherches dues a M. Saint-Lager, il ressort que la clispersion de certaines de ces espèces est sous linfluence de la juroportion plus ou moins grande de potasse que renferme le sol. II en serait ainsi du Sarothamne, qui croit dans les sols siliceus et peut s'accommoder d'un sol faiblement calcaire, pourvu qu'il contienne une qrantite suffisante de polasse; jai constaté des faits qui viennent à l'appui de cette explication dans beaucoup de points des Coteaux du Rhône, ou les allurions glaciaires renferment de nombreux galets feldspathiques dont la decomposition introduit dans le sol l'alcali qui parait indispensable à la végétation de certaines plantes. Mais comme des analyses faites a ce point de vue me manquent encore, je crois devoir renvoyer lexamen de celte question à plus tard.

${ }^{2}$ Le Leucobryum ylaucum existe aussi dans le Dauphiné et dans le Bugey, près de Belley, au bois de Chazey : jai vérifie qu il y croit de mème sur le terrain erratique qui_recouvre la mollasse. 
\$3. - Énumération systématique des végétaux

constituant la Floredes vallées et bas-plateaux du Lyonnais

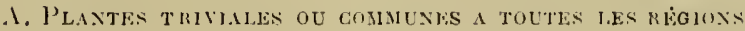

DE LA FLORF, LYONNUSE 1

Clematis Vitalba, Ranunculus aquatilis, R. arris, R. repens, R. Jullosus, R. arvensis, R. sceleratus, Ficaria ranunculoides, Caltha palustris.

Papaver Rhæas, Chelidonium majus, Fumaria officinalis.

Raphanus Raphanistrum, Sinapis arvensis, Barbarea vulgaris, Sisymbriun officinale, S. Alliaria, Nasturtium officinale, N. silvestre, Turritis glabra, Arabis Thaliana, Carlamine pratensis, G. hirsuta, Alyssum calycinum, Draba verna, Roripa amphibia, Thlaspi arvense, Capsella Bursa-pastoris, C. rubella, Lepidium campestre, L. graninifolium.

Hélianthemum vulgare, H. procumbens.

Viola hirta, V. odorata, V. tricolor. - Resella sp.. - Polygala vulgaris.

Silene inflata, S. dioica, S. nutans, Iychnis Flos-Cuculi, L. Githago, Saponaria officinalis, Dianthns prolifer, D. Carthusianorum, Alsine tenuifolia, Arenaria serpyllifolia, Stellaria media, St. Holostea, Holosteum umbellatum, Cerastium glomeratum, brachypetalum, senidecandrum, obscurum, triviale.

Linum catharticum.

Malva silvestris, M. rotundifolia.

Geranium columbinum, G. dissectum, G. molle, G. pyrenaicum, G. rotundifolium, G. Robertianum, Erodium cicutarium.

Hypericum perforatum.

Acer campestre.

Evonymus europreus, Rhammus cathartica.

Ononis repens, Nedicago Lupulina, falcata, melia, sativa, maculata, minima, Trifolium pratense, fragiferum, arvense, repens, micranthum, minus, procumbens, Lotus corniculatus, Astragalus Glycyphyllos, Vicia sativa, V. an gustifolia, V. sepium, V. Cracca, V. tenuifolia, V. hirsuta, V. tetrasperma, Lathyrus pratensis, Coronilla varia, Onobrychis sativa.

I'runus spinosa, insititia, Cerasus avium, vulgaris, Spircea Ulmaria, Geum u'banum, Potentilla Fragaria, P. verna, P. reptans, P. Anserina, Fragaria vesca,

1 Cette énumération, donnée une fois pour toutes, ne sera pas répétée pour les au tres régions. 
Rujus discolor, cæsius, Rosa sp.' , Agrimonia Eupatolia, Poterium Sanguisorba, Sanguisorba officinalis, Alchemilla arvensis, Mespilus germanica, Cratrgus oxyacantha, C. oxyacanthoides, Pirus communis, Malus communis, M. acerba. Epilobium montanum, parviflorum, hirsutum, Callitriche stagnalis, Lythrun Salicaria.

Bryonia dioica. - Portulaca oleracea.

Herniaria hirsuta, Scleranthus annuus.

Sedum Telephium, album, acre, reflexum

Ribes Uva-crispa, R. rubrum.

Saxifraga granulata, S. tridactylites.

Daucus Carota, Torilis Anthriscus, T. segetum, Angelica silvestris, Pastinaca opaca, Heracleum Sphondylium, Silaus pratensis, Etlınsa Cynapium. Ananthe Phellandrium, Buplcurum falcatum, Pimpinella Saxifraga, Egopodium Podagraria, Petroselinum segetum, Anthriscus vulgaris, $\Lambda$. silvestris, Chærophyllum temulum, Eryngium campestre.

Hedera Helix, Cornus sanguinea.

Viscum album.

Simbucus Ebulus, s. nigi'a, Viburnum Lantana, V. Opulus, Loniccra Perielymenum, L. Xylosteum.

Galium Gruciata, verum, elatum, erectum, Aparine. Asperula Cynanchica.

Valeriana officinalis, V. dioica, Valerianclla olitoria, carinata, Amricula. Dipsacus silvestris, Knautia arvensis, Scabiosa Columbaria.

lispatorium cannabinum, Tussilago Farfara, Solidago Virga-aurea, Erigeron canadensis, E. acris, Bellis perennis, Senecio vulgaris, S. Jacobæa, Aptemisia vulgaris, A. campestris, Lencanthemum vulgare, Matricaria inodora, Anthemis arvensis, Achillea Millefolium, A. Ptarmica, Bidens tripartita, Inula Conyza, Pulicaria dysenterica, Onopordon Acanthium, Cirsium lanceolatum, palustre, arvense, Carduus nutans, Centaurea Jacea, C. Cyanus, C. Scabiosa, C. Calcitrapa, Carlina vulgaris, Lappa minor. Cichorium Intybus, Lampsana communis, Leontodon autumnalis, Picris hieracioides, Tragopogon pratensis, Taraxacum officinale, Lactuca Scariola, Sonchus oleraceus, S. asper, Crepis taraxacifolia, fotida, virens, Hieracium Pilosella, H. Auricula, H. murorum, H. vulgatum.

Phyteuma spicatum, Specularia Speculum, Campanula glomerata, C. Trachelium, C. rotundifolia.

Utricularia vulgaris.

Primula officinalis, Lysimachia vulgaris, L. Nummularia, Anagallis phœnicrea, A. cærulea.

1 Il a paru inutile, dans cette liste comme dans les listes speciales, de citer les espèces encore si peu connues (surtout clans leur dispersion) des genres Rubus, Rosa, Hieracium, etc. 
Fraxinus excelsior, Ligustrum vulgare, Vinca minor, Erythræa Centaurium.

Convolvulus sepium, C. arvensis, Cuscuta sp.

Symphytum officinale, Lycopsis arvensis, Lithospermum officinale, L. ar vense, Echium vulgare, Pulmonaria tuberosa, Myosotis hispida, M. intermedia, Cynoglossum officinale, Heliotropium europeum.

Lycium Barbarum, Solanum Dulcamara, S. nigrum, Datura Stramonium, Hyoscyamus niger, Verbascum sp.

Scrofularia nodosa, S. aquatica, S. canina, Antirrhinum Orontium, Linaria spuria, L. vulgaris, Veronica Chamœdrys, V. Beccalunga, V. Anagallis, V. offi-. cinalis, arvensis, serpyllifolia, triphyllos, agrestis, polita, hedercefolia, Euphrasia officinalis, Olontites verna, Rhinanthus hirsuta, Rh. minor.

Orobanche sp.

Mentha sp. Lycopus europæus, Origanum vulgare, Thymus Serpyllum, Caiamintha Acinos, C. Clinopodium, Salvia pratensis, Glechoma hederacea, Lamium sp., Stachys palustris, S. recta, Betonica officinalis, Ballota foetida, Marrubium vulgare, Brunella vulgaris, B. alba, Ajuga reptans, A. genevensis, Teucrium Scorodonia, T. Chamæedrys.

Verbena officinalis.

Plantago officinalis, media, lanceolata.

Amarantus sp., Atriplex sp., Chenopodium sp.

Rumex pulcher, obtusifolius, cong lomeratus, Acetosa.

Polygonum lapathifolium, P. Persicaria, P. Hydropipes, P. mite, P. minus, P. aviculare, P. Convolvulus, P. dumetorum.

Euphorlia Helioscopia, platyphyllos, stricta, dulcis, verrucosa, Cyparissias, c.igua, Peplus, amygdaloides, Lathyris, Mercurialis annua.

Ulmus campestris.

Urtica ureus, U. dioica, Parietaria officinalis, Humulus Lupulus.

Fagus silvatica, Quecus Robur, Corylus Avellana, Carpinus Betulus, Salix alba, amygdalina, purpurea, capræa, Populus tremula, alba, nigra, Betula pendula, Alnus glutinosa.

Alies pectinata, Juniperus communis.

Alisma sp. - Colchicum autumnale.

Ornithogalum umbellatum, Allium sp., Muscari, comosum, M. racemosum, Polygonatum vulgare, P. multiflorum, Convallaria ma jalis, Tamus communis.

Iris pseudo-acorus.

Ol'chis laxiflora, latifolia, maculata, Conopea.

Potamogeton sp., - Lemna sp. - Typha sp.

Sprarganium sp. - Arum maculatum.

Juncus conglomeratus, effusus, glaucus, lamprocarpus, acutiflorus, compressus.

-- Luzula Forsteri, pilosa, campestris, maltiflora.

Eriophorum latifolium, angustifolium - Scirpus silvaticus, lacustris, palustrsi, 
- Carex disticha, vulpina, muicata, leporina, rulgaris, glauca, præcox, silvalica, Ederi, vesicaria, paludosa, riparia, hirta.

Phleum pratense, Alopecurus pratensis, A. agrestis, Setaria glauca, viridis, verticillata, Panicum Crus-Galli, P. sanguinale, Cynodon Dactylon, Andropogon lschoemum, Phragnites communis, Agrostis alba, Milium effusum, Avena pubescens, A. pratensis, Arrhenaterum elatius, Trisetum flavescens, Holcus lanatus, Glyceria fluitans, Poa amna, nemoralis, bulbosa, compressa, pratensis, trivialis, Briza media, Cynosurus cristatus, Festuca duriuscula, rubra, pratensis, Bromus tectorum, sterilis, crectus, arvelsis, mollis, racemosus, Hordeum murinum, Agropyrum glancum, repens, caninum, Brachypodium silvaticun, B. pinnatum, Lolimm perenne, L. temmlentun, Polypodium vulgare, Polystichum Filix-mas, Aspidium Filix-femina, Asplenium Trichomanes, A. Ruta-muraria, A. Adian. tum-nigi'ums.

Equisetum arvense, palustre, limosum.

Chara sp.

B. Plantes molns comaunes, ples répandufs

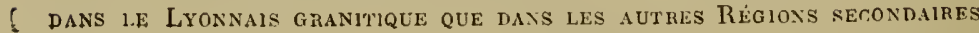

- LA PLUPART INDIFFERENTES

QUELQUES-UNES CALCICOLES, DAUTRES SILICICOLES.

Anemone nemorosa, A. ranunculoides, A. rubra Jord. Ranunculus confums, R. Flammula, R. auricomus, R. nemorosus, R. monspeliacus, R. Chærophyllos, R. parvilorus, Helleborus foetidus, Aquilegia vulgaris, Isopyrum thalictroides.

Papaver dubium, P.Argemone, Corydalis solida, Hesperis matronalis, Barbarea præcox, Cardamine impatiens, Draba muralis, Roripa nasturtioides, Lepidium ruderale, Senebiera Coronopus, Bunias Erucago.

Parnassia palustris.

Saponaria Vaccaria, Sagina procumbeus, S. patula, Mohringia trinervia, Stellaria graminea. - Elatine hexandra, E. Alsinastrum.

Malva Alcea. - Geranium pyreuaicum.

Hypericum hirsutum, montanum.

Acer monspessulanum.

Impatiens Noli-tangere, Oxalis Acetosella, stricta.

llex Aquifolium.

Genista sagittalis, pilosa, tinctoria (Anthyllis vulneraria), Medicago apiculata, M. Gerardi, Trifolium ochroleucum, striatum, glomeratum, Lagopus, Trigc.nella monspeliaca, Lotıs major, Vicia monanthos, Lathyrus Aphaca, Nissolia, hirsutus, niger, angulatus, sphæricus Orobus tuberosus, Hippocrepis comosa. 
Spiræa Filipendula, Potentilla micrantha, rupestris, Rulus glandulosus, R. tomentosus, R. thyr'soideus, Rosa sp., Alchemilla vulgaris, Sorbus torminalis, Amelanchier vulgaris.

Epilobium obscurum, E. tetragonum, E. spicatum, Isuardia palustris, Circæa lute. ana, Myriophyllum sp., Ceratophyllum sp., Callitriche vernalis, Herniaria glabra.

Sedum Ceprea, S. ruhens, S. sexangulare, Sempervirum tectorum.

Petcedanum carvifolia, P. Oreoselinum, Tordylium maxinum, Enanthe pencedanifolia, CE. fistulosa, Bupleurum affine, tenuissimum, Carum Carvi, Heloscialium nodiflorum, Scandix Pecten. Sanicula europæa.

Adoxa Moschatellina.

Galium silvestre, montanum, palustre, uliginosum, Asperula odorata. - Scabiosa Succisa.

Senecio viscosus, erraticus, flosculosus, Leucanthemum corymbosum, Parthenium, Gnaphalium silvaticum, Filago germanica, Cirsiun eriophorum, Centaurea intermedia, Serratula tinctoria, Hypochæris maculata, H. radicata Leontodon hispidus, Chondrilla juncea, Lactuca saligna, I. muralis, Prenanthes purpurex, Crepis paludosa, Crepis sctosa, Pterotheca nemausensis,

Campanula Rapunculus, C. persicifolia.

Primula grandiflora, P. variabilis, Lysimarhia nemorum.

Gentiana Pneumonanthe, Menyanthes trifoliata.

Anchusa italica, Lithospermum permixtum, Myosotis palustris, stricta, silvatica.

Verbascum montanum, Linaria ochrolenca, L. minor, Gratiola officinalis, Digitalis lutea, D. grandiflora, Euphrasia nemorosa, Pedicularis palustris, Melampyrum pratense.

Nepeta Cataria, Galeopsis Tetrahit, Stachys silvatica, Melitis Melissophyllum Scutellaria galericulata, Sc. minor', Teucrium Botrys.

Plantago arenaria, Armeria plantaginea.

Pinus silvestris.

Salix fragilis, cinerea, aurita.

Scilla bifolia, Phalangium Liliago, Paris quadrifolia, Maianthemum bifolizm.

Epipactis ovata, Orchis ustulata.

Arum italicun.

Carex pulicaris, Schreberi, pallescens, panicea, C. stellułata.

Alopecurus geniculatus, fulvus, utriculatus, Agrostis Spica-venti, Kœehleria cristata, P'oa megastachya, Melica ciliata, M. uniflora, Bromus giganteus.

Ceterach officinarum, Aspidium aculeatum, Polystichum spinulosum, Cystopteris fragilis, Asplenium Hulleri.

Observations. - Sur ces 185 espèces, 35 méritent une mention spéciale à cause de leur dissémination moins générale. Ce sont : Pulsatilla rubra Jord., Anemone ranunculoides, Ranunculus 
monspeliacus, R. Chrrophyllos, Isopynum thatictroides, Corydalis solida, Hesperis matronalis, Cardamine impaliens, Draba muralis, Senebiera Coronopus, Saponaria Vaccaria. Acer monspessulanum, Impatiens Noli-tangere, Rubus glan.. dulosus, tomentosus, Alchemilla vulgaris, Peucedanum parisiense, Carum Carvi, Adoxa Moschatellina, Senecio flosculosus, Gnaphalium silvaticum, Centaurea intermedia, Prenanthes purpurea, Crepis paludosa, Lysimachia nemorum, Gentiana Pneumonanthe, Menyanthes trifoliata, Teucrium Botrys, Paris quadrifolia, Maianthemum bifolium, Alopecumes fulvus, Polystichum spinulosum, Cystopteris fragitis, Asplenium Halleri.

Les unes sont des plantes méridionales qui remontent jusqu’ici dans des terrains secs ou des expositions chaudes, comme : Ta nunc. Chrophyllos, R. monspeliacus, Draba muralis, Sc nebiera Coronopus, Acer monspessulanum, Teucrium Botrys; - les autres habitent les vallées ombragées ou les bois courerts : An. ranunculoides, Isop.thalictroides, Curdamine impatiens, Impatiens Noli-tangere, Adoxa Moschatellina, Senecio flosculosus, Prenanthes pumpurea, Lysimachia nemorum, Pairis quadrifolia, Maianthemum bifolium, etc.; leur centre de dispersion parait être la région montagneuse.

$\mathrm{Au}$ point de vue de leur rapport avec la nature du sol, 51 de ces espèces sont données comme silicicoles par Lecoq; aucune n'est indiquée comme calcifuge par M. Contejean ${ }^{1}$.

Sur ces 51 espèces (réputées silicicoles par Lecor), les Anemone ranunculoides, Isopyrum thalictroides, Barbarea precox, Oxalis Acetosella, Carum Carvi, Sanicula europra, Ranun culus auricomus, Asperula odorata, Paris quadrifolia, Maianthemum, sont pour nous indifférentes, parce qu'on les rencontre non seulement dans le Lyonnais, mais encore dans les prairies et l'humus des bois et des forêts du Jura; il est vrai que si on les admettait comme silicicoles, on pourrait expliquer leur

1 LEcoe. Études sur la géogr. bot. de l'Europe. 185̌́, t. II, p. 67 et seq. - CoNtFjean. De l'influence du terrain sur la végétation (Ann. Sc. natur., 5" sér., t. XX, 1874, p. $266 ; 6^{\circ}$ série, t. II, 1875, p. 222 et seq.) 
présence dans les réģions calcaires par la nature spéciale des stations dans lesquelles elles croissent; on sait, en effet, que même sur un sous-sol calcaire, le sol des prairies et l'humus des forêts ne contient que très peu de carbonate de chaux et constitue soulvent un sol exclusivement siliceux ${ }^{1}$.

Il n'en est pas de même des Aquilegia vulgaris, Paparer dubium?, Genista pilosa, tinctoria, Trifolium ochroleucum, striatum, glomeratum, Potentilla rupestris, Rubus glandulosus, Sorbus torminalis, Herniaria glabra, Sectum Cepra. S. rubens, Cirsium eriophomm, Seratula tinctoria, Hypochceris maculata, Linaria minor, Digitalis grandiflora, $P_{0}$ lystichum spinulosum, Asplenium Halleri, qui sont domnèes aussi comme silicicoles par M. Lecoq. Ces espèces croissent aussi bien sur les roches calcaires et les pelouses du Mont d'Or, des coteaux du Rhône et du Jura que sur les roches silicatées et les pelouses du Lyonnais proprement dit.

La même observation s'applique aux espèces suivantes à stations bien diverses, cultures, lieux humides, qui dans notre régio:1 sont tout à fait indifférentes, bien qu'elles soient un peu plus communes dans le Lyonnais : Corydalis solida, Stellaria graminea. Saponaria Vaccaria, (Sagina procumbens), Mahringia triner.. nia, Hypericum hirsutum, Oxalis stricta, Vicia monanthos, Lathyrus niger, angulatus, Scabiosa Succisa, Senecio iscosus, Gnaphalizm silvaticum.

$\mathrm{Si}$, dans notre liste $\mathrm{B}$, quelques espèces ont été considérées, it tort, ainsi que nous venons de le voir, comme préférant les terrains siliceux, d'autres ont été indiquées, par contre, comme calcicoles. Je ne m'arrêterai pas aux Ranunc. Chærophyllos, R. par'vifloms, Malva Alcei, Medicago apiculata, M. Gerardi, Lathymus Aphaca, L. sphrricus, données comme telles par Lecoi, les assertions de ce phytostatisticien ne reposant pas sur un examen assez critique des localités; mais d'autres espèces de notre

1 Voy. par ex. dans Ogérien (Hist. natur. du Jura, t. I, p. 208), des analyses des sols des forêts du Jura renfermunt 14 à $280 / 0$ de silice et seulement 1 à $40 / 0$ de calcaire. 
énumération, les Helleborus foelidus, Hypericum hirsulum, Anthyllis Vulneraria, Digitalis lutea, Melica ciliata et Asplenium Halleri, sont des plantes calcicoles pour M. Contejean ${ }^{1}$, et l'autorité de ce botaniste exige que nous examinions avec plus de soin leur dispersion géographique dans notre région.

Si l'Helleborus fretichus est très répandu dans les sols calcaires du Mont-d’or, des coteaux du Rhòne, on le trouve non moins abondamment sur tous les éboulis gneissiques des vallées du Lyonnais, comme on peut le voir a Rochecardon, Tassin, Francheville, Brignais, etc.

Quoique moins fréquent, l'Hypericum hirsutum se rencontre assez souvent dans les bois recouvrant les gneiss de Tassin, Francheville, dans les monts du Lyonnais, etc.

L'Anthyllis Vulneraria serait peut-être un peu plus préférent; car, si on le rencontre sur toutes les pelouses sèches de nos environs, quelle.que soit la nature du sol, il est certainement plus fréquent sur les pelouses calcaires du Mont-d'Or, des coteaux du Rhône, de Crémieu et du Jura.

De même pour le Digitalis lutea, qui est encore plus spécial aux sols calcaires; cependant il a été rencontré dans quelques points de la région siliceuse du Lyonnais; du reste, M. Legrand l'indique sur les granites (et les basaltes) dans le Forez ${ }^{2}$.

Quant au Melica ciliata, la question de son adaptation à certains sols se complique d'une discussion de synonymie; sous ce nom de $M$. ciliata, il est certain qu'on a confondu plusieurs formes, voisines des M. Magnolii, nebrodensis, etc.; il est possible que quelques-unes d'entre elles préfèrent les sols siliceux et l'autres les terrains calcaires. Quoi qu'il en soit, on trouve dans les environs de Lyon, sur les granites et les gneiss, par exemple à Oullins, Saint-Genis-Laval, etc., un Melica ciliata ou Melica ciliata Vill.? M. nebrodensis Gr. God. non Parl., M. glauca Fr. Sch. sec. Car. Et. cles Fl., $6^{\bullet}$ éd., 1879, t. II, p. 824 (excl. $M$. $M a-$ gnolii Gr. (God.), qui paraît identique à celui que j'ai observé sur'

- Ann. des Sc, natur.,loc:cit.

2 Statist. botan. du Forez, p. 188. 
les calcaires de Couzon et du Bugey; je l'ai retrouvé plus loin encore dans la région granitique, sur les schistes carbonifériens (cornes vertes et cornes rouges) de l'Arbresle et de Sain-Bel, dont le tapis végétal est constitué, comme nous le verrons plus tard, par des espèces tout à fait silicicoles.

Reste l'Asplenium Halleri, que nous avions nous- même considéré longtemps comme éminemment calcicole, tellement il nous avait paru contrastant dans le Jura et dans le Bugey, mais il suffit de visiter les rochers du Garon, où on peut le cueillir en quantité notable sur le granite et le gneiss, en société de l'Asplenium sep tentrionale, pour se convaincre qu'il n'est pas aussi exclusivement calcicole qu'on le croit généralement.

Sur ce mème sujet, nous tenons aussi à liare remarquer que si nous avons mis dans notre liste $\mathrm{A}$, renfermant les plantes triviales ou communes à toute la Flore lyonnaise, le Teucrium Chamadrys, qui est aussi donné comme calcicole par M. Contejean, c'est que son abondance sur les granites de nos environs, à Oullins, par exemple, nous empêche de le regarder comme caractéristique des sols calcaires '.

\section{Plintes PRÉfirant les Sols SILIClud \\ 1. Silicicoles peu adhélentes :}
Diantlus Armeria.
Alsine viscosa.
Nalva moschata.
Geranium nodosun.
(Rhamnus Frangula).
Genista anglica.
G. germanica
Trifolium arvense.
T. scalrum.
(T. subterıaneum).
T. filiforme.
Vicia lutea.
Cerasus Padus.
Potentilla argentea.
Rubus discolor.
Epilobium lanceolatum.

1 Du reste, noas devons reconnaitre que M. Contejean arait déjà constaté que certaines des espèces qu'il donne comme calcicoles, entre autres les Helleborres forticlus Teucrium Chamadrys, etc., qui font précisement l'objet de nos obsel'vations, pouvaient se trouver cóte à côte des calcifuges, dans des terrains dépourvus de carbonale de chaux, le diluvium, par exemple (Ann. sc. natur., 1875, t. II, p. 252). De cestail.s et d'autres analogues, M. Contejean conclut que les calcicoles sont moins exclusives que les silicicoles. 
Sedum maximum.

S. elegans.

(Saxifraga granulata). (Peucedanum Oreoselinum).

Conium maculatum.

Crucianella angustifolia.

Senccio silvaticus.

S. er'raticus.

Chamomilla nobilis.

Filago germanica.

Centaurea amara.

C. Duboisii.

Thlincia hirta.

Hicracium Pilosella.

H. umbellatum.

Jasione montana.

Campanula patula.

Symphytum tuberosum.

Linaria Elatine.

L. striata.

L. Pelliceriaua.

Veronica nontana.

V. acinifolia.

Pedicularis silvatica.
Galeopsis Tetrahit.

Polycnemum minus.

Rumex Acetosella.

Quercus Robur.

Pinus silvestris.

Orchis Morio.

O. ustulata.

O. coriophora.

0 . viridis.

Carex remota.

C. polyrhiza.

Anthoxanthum odoratum.

Agrostis vulgaris.

A. Spica-renti.

Holcus mollis.

Danthonia decumbens.

Festuca ovina.

$\mathrm{F}$. temuifolia.

F. heterophylla.

F. sciuroides.

Gaudinia fragilis.

Lolium rigidun.

Nardurus Poa.

Quelques- unes de ces espèces sont considèèes comme nettement calcifuges par M. Contejean; ce sont, par exemple : Potentilla argentea, Jasione montana, Rumex Acetosella, Carex remota, et Nardurus Poa.

2o Silicicoles des lieux humides, marécageux ou tourbeux

Ranunculus philonotis.

R. liederaceus.

Roripa pyrenaica.

Drosera rotundifolia.

Sagina procumbeus.

Sagina apelala.

s. patula.
Stellaria uliginosa.

Cerastium erectum.

Hypericum tetrapterum.

Callitriche lamulata.

Montia rivularis.

Hclosciadium inundatum.

Bunium verticulatum. 
Senecio aquaticus.

Bidens cernuus.

Taraxacum palustre.

Grepis paludosa.

Myosotis lingulata.

Gratiola officinalis.

Mentha Pulegium.

Scutellaria minor.

Sc. galericulata.

Molinia cærulea.
Ophioglossum vulgatum.

Littorella lacustris.

Rumex maritimus.

Sparganium simplex.

Juncus pygmæus.

J. capitatus.

J. Tenageia.

Scirpus supiutus.

Sc. setaceus.

Heleocharis acicularis.

Les Ranunculus hedleraceus, Drosera rolundifolia, Cerastium erectum. Montia rivularis, Juncus Tenageia sont données c)mme calcifuges par M. Contejean; dans notre région, les $R$. hedleraceus, Montia rivularis le sont en effet, très-nettement; ces espèces ne viennent que dans les eaux des terrains granitiques; mais le:s Drosera, Jincus Tenageia, etc., sont comme les Ophioglosses, Gratiole, Biclens cermua, etc., des espèces qui croissent da:is les régions calcaires aussi bien que dans les granitiques, pourvu qu'elles trourent un sol tourbeux, lequel est, il est vrai, généralement dépourvu de carbonate de chaux ${ }^{1}$. C'est pour cette raison que nous avons cru devoir former un groupe a part de ces silicicoles, qui ont, du reste, un habitat spécial.

30 Silicicoles des terrains argilo-siliceux

Myosurus minimus.

Gypsophila muralis.

Spergularia segetalis.

Sp. rubra.

Lotns tenuis.

L. diffusus.

Agrimonia clorata.
Filago arvensis.

F. gallica.

Centunculus mininus.

Cicendia filiformis.

Lythrum Hyssopifolia.

Peplis Portula.

Corrigiola littoralis.

1 Eu attendant que nous traitions de la Flore des Tourbières du Lyonnais, voici des analyses de prés tourbeux du Jura: - $\mu^{\circ} 1$ : silice 19.4 , calcaire 2.7 ; no 2 : silice 24.2, calcaire 0.2, p. 0/0. (Ogélien, ibid., p. 212). 
Pulicaria vulgaris.

Gnaphalium luteo-album.

Gn. uliginosum.

Filago montana.
Stachys arrensis.

Spiranthes autumnalis. Juncus bufonius.

M. Contejean considère comme calcifuges seulement les Myosurus minimus, Spergularia rubra, Agrimonia odorata, Corrigiola littoralis, Filago montana; dans notre région et aussi, comme nous le verrons plus tard, dans les régions voisines du Forez et de la Dombes, les autres espéces que nous avons groupées dans cette catégorie sont pour nons caractéristiques au même degré ; leur dispersion a une importance très grande dans l'étude de cette question de relation du sol avec la régétation; en effet, leur habitat dans un sol argileux, devenant souvent très compacteà la suite des sécheresses, est un argument puissant contre la doctrine de l'influence physique, ces especes pourant prospèrer, comme nous l'avons observé souvent pour le Corrigiola littoralis, aussi bien dans les terrains meubles et les sables, que dans les sols compactes, argileux, pourvu qu'ils soient siliceux ${ }^{1}$.

$4^{\circ}$ Silicicoles des rochers. sables, pelouses seches, granitiques ou gueissiques.

* Sinapis Cheiranthus.

* Teesdalia nudicaulis.

Thlaspi silvestre Jord.

Dianthus deltoides.

* Silene Armeria.

Viscan ia purpureal.

Spergula arven:ir.

Sp. Morisonii.

* Sp. pentandra.

* Polygala depressa.

* Hypericum humifusum.

$\star$ H. pulchrum.

* Ulex europæus.

*U. nanus.
* Sarothamnus scoparius.

Trifolium elegans.

T. Lagopus.

Vicia lathyroides.

Orobus tuberosus.

^ Ornithopus perpusillus.

Rosæ (Gallicanæ).

Potentilla Tor'mentilla.

Epilobium collinum.

* Montia minor.

* Scleranthus perennis.

* Umbilicus pendulinus.

Sedum maximum.

* Senecio adonidifolius.

1 Cr. observations analogues de M. Saint-Lager; daus $A n n$. Sor. botan. de Lyon. 
Matricaria Chamomilla.

* Centaurea nemoralis.

Arnoseris minima.

* Hypochœris glabra.

Scorzonera humilis.

Andryala sinuata.

* Jasione Carioni.

* Calluna vulgaris.

* Myosotis versicolor.

M. Balbisiana.

Anarrhinum bellidifolium.

Linaria arvensis Desf.

Veronica verna.

* Digitalis purpurea.

* Galeopsis dubia.

Plantago carinata.

* Castanea vulgaris.
Luzula silvatica.

Carex pilulifera.

Mibora verua.

* Corynephorus canescens.

* Aira caryophyllea.

A. aggregata.

A. patulipes Jord.

A. elegans Gaud.

* A. præcox.

* Deschampsia flexuosa.

Avera temuis.

* Festuca psendomyuros.

* Nardus stricta.

* Asplenium septentrionale.

* A. germanicum.

Pteris aquilina.

Toutes les espèces précédées d'un astérisque sont des calcifuges pour M.Contejean; on voit donc que nos deux séries offrentla plus grande analogie; les quelques espèces que nous donnons comme silicicoles caractéristiques et qui ne se trouvent pas dans l'énumé ration établie par le savant professeur de Poitiers sont, ou des espèces critiques négligées par un grand nombre de botanistes, comme Thlaspi silvestre Jord., Spergula Morisonii Bor., Myosotis Balbisiana Jord., Aira aggregata Timeroy, A. painlipes Jord., ou des espèces rares dont la dispersion est généralement peu ètudièe, comme Trifolium elegans, T. Lagopus, Linaria ar. vensis, Aira elegans, Avena tenuis, etc., mais que nos recherches ne nous ont jamais montrées ailleurs que dans les terrains siliceux. Il en resle quelques unes qui demandent quelques mots d'explication; ce sont les Potentilla Tomentilla, Scorsonera humilis, Luzula silvatica, qui, très abondantes dans la région granitique, se retrouvent cependant dans les montagnes calcaires; mais c'est alors dans un habitat spécial, sur un sol siliceux, le Polentilla Tormentilla dans les tourbières desséchées, les Scorzonera humilis et Luzula silvatica dans l'humus siliceux des 1 rairies ou des forèts; du reste, le mème fait se produit pour les Dianthus deltoides, Polygala depressa, Nardus stricta, qu'on 
peut observer dans les pelouses des montagnes jurassiques et qui sont cependant considérées, avec raison, comme nettement calcifuges par M. Contejean.

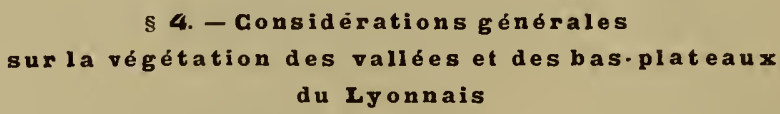

La végétation d'une région demande à être étudiée dans sa composition intime et dans ses rapports avec celle des contrées voisines ou analogues; nous allons donc donner les caractères de la Flore du Bas-Lyonnais et étudier ses rapports avec la Flore des régions qui l'entourent.

\section{A. Caractéles de la Flori:}

Ces caractères peuvent se grouper d'après les diverses influences qui agissen $t$ sur la distribution des végétaux, climat, exposition, influence chimique ou physique du sol, de voisinage, d'altitude, etc.

\section{$l^{\circ}$ Influence chimique du sol: Flore silicicole}

Il est superflu sans doute d'insister maintenant sur ce fait, mis en pleine évidence par les nombreuses énumérations de plantes qui précèdent, que la Flore des vallées et bas-plateaux du Lyonnais est composée d'espèces dont la plupart, les plantes triviales ou indifférentes exceptées, paraissent préférer dans les autres contrées les sols siliceux aux sols calcaires ${ }^{1}$; de même nous avons ru, avec la même clarté, que quelles que fussent les variations nombreuses d'état de désagrégation présentées par les granites, les gneiss et les alluvions anciennes, ces roches et le sol végétal qui en prore-

1 . Yous tenons à répéter de nouveau que nous ne pretendons pas expliquer a quoi est due cette influence prépondérante de la composition chimique du sol, mais que uous constatons seulement cles fuits 
naient pouvaient être considerès comme formés par des éléments siliceux et alcalins prédominants et dépourvus à peu près complètement de carbonate de chaux. C'est donc à l'influence de la composition chimique du sol et non à son état pliysique qu'on doit provisoirement attribuer cette association d'espèces caractéristiques qui se groupent autour des Sarothamne, Châtaignier, Polytrichs et Lécidée géographique.

$2^{\prime}$ Influence physique. - Variété des milieux et des stations

Un autre caractère de la Flore de cette région, c'est la variété de stations des végétaux; en effet, ainsi qu'on peut s'en rendre compte en parcourant les exemples d'herborisations choisis dans les points les plus divers de la région, et les listes des espèces groupées d'après leurs stations naturelles, tous les habitats principaux qu'on observe dans l'intérieur du continent sont ici représentés; on peut donc y étudier la végétation des rochers, des graviers, des sables et des éboulis, des sols argileux, des cultures, des bruyères, des bois, des pelouses, des prairies marécageuses, des eaux courantes, etc. On verra que cette variété de stations ne se retroure pas dans les autres régions de la Flore lyonnaise.

30 Influence du climat et de l'exposition:

Contraste entre les deux versants des vallèes; especes mériliouales: différence entre la végétation des vallèes méridionales et septentrionales.

Devant réunir tout ce qui concerne la climatologie végétale du Lyonnais dans un chapitre spécial, nous ne parlerons ici que de l'influence de l'exposition. Elle se fait sentir d'une manière très nette le long des vallées dirigées de l'O. à l'E. ; en effet, dans la première partie de leur cours, qui affecte généralement cette direction, par exemple le Charbonnières et le Ratier jusqu'au Pontd'Alaï, l’Iseron jusqu'à Francheville, le Garon jusqu'à Brignais, 
etc., leurs deux versants présentent le plus grand contraste dans leur végétation; le versant qui regarde le $\mathrm{N}$., généralement boisé, abrite des plantes des lieux frais, humides, des espèces plus ou moins hygrophiles comme :

Ranunculus auricomus.

Anemone ranunculoides.

Isopyrum thalictroides.

Cardamine impatiens.

Møhringia trinervia.
Acloxa Moschatellina.

Umbilicus pendulinus.

Lactuca muralis.

l)igitalis purpurea.

Fèstuca heterophylla, etc.

de nombreuses Fougères: Aspidium aculeatum, A. Filix-ficmina, Polystichum Filix-mas, Cystopteris fragilis, etc., des Mousses et des Hépatiques, etc.

Le versant tourné versle S. est, au contraire, aride et couvert de plantes xérophiles, Sedum, Saxifraga, Silene, Ranunculus Charophyllos, R. monspeliacus, de Spergules, Genêts, Jasiones, Linaire, Aira, Vulpia, etc., et quelques especes du Midi de la France.

Mais à ce dernier point de vue, on peut diviser notre région en deux zones : l'une méridionale, s'étendant de Givors au Garon, l'autre septentrionale, occupant le reste de la surface jusqu'à l'Azergue.

$1^{\circ}$ Zone méridionale. - Elle comprend les vallées du Garon et de ses affluents, la vallée du Mornantet, dont la végetation se rattache à celle du Gier et des environs de Condrieu et de Vienne. Cette végétation est caractérisée par la présence de quelrues plantes du Midi de la France, croissant ici sur les rochers ou dans les sables bien exposés au soleil; les principales de ces espèces sont :

Lianunculus moinspeliacus: Vallées du Gier, du Mornantet, du Garon;

Draba muralis: V. du Gier et du Mornantet;

Acer monspessulanum: V. dn Gier et du Garon;

Silene Armeria: V. du Gier, du Mornantet et du (iaron;

Trigcnella monspeliaca: sur la limite de la région à Beaunant, Tassin;

Trifolium Layopus: Givors, vallée du Garon;

Crucianella angustifolic: V. du Gier, du Garon; 
Centaurea tenuisectc Jord.: V. du Gier, du Mornantet;

Bartramia stricta: Orliénas (sec. Debat), etc.

Du reste, les espèces méridionales qui remontent dans les rallées du Bas-Lyonnais sont rares en comparaison du nombre de ces plantes qu'on peut observer dans l'autres parties de la vallée du Rhône, sur les coteaux qui bordent ce fleuve et jusque dans le massif du Mont-d'Or. Si l'on prend garde que, dans le sol de ces dernières régions, c'est l'élément calcaire qui domine (ainsi que nous le verrons plus tard), on sera tenté de conclure que, en outre de l'exposition, la nature du sol peut avoir une certaine influence sur la dispersion de ces espèces; on sait, en effet, que les sols calcaires sont plus chauds que les sols silicenx, et Lecoq avait déjà observé que les espèces méridionales s'avancent plus vers le $\mathrm{N}$. sur les calcaires des causses, que sur les granites et les gneiss ${ }^{1}$. Nons reviendrons du reste sur ce sujet, à propos de la Flore du Montd'Or, des coteaux du Rhône, et dans les considérations générales que nous donnerons sur la Flore méridionale de la région lyonnaise ${ }^{2}$.

$2^{\circ}$ Zone septentrionale. - Ies vallées de l'Iseron, du Charbonnières, etc., ne renferment pas ces espèces des régions plus chaudes; leur végétation se rapproche au contraire de celle des rallées des montagnes, on de la vallée de l'Azergue, comme le montrent Veronica montana, Stachys alpina, Paris quadrifolia, etc. et surtout :

Impatiens Noli-tangere.

Lychnis silvestris.

Cardamine impatiens.
Geranium nodosum.

Senecio silvaticus, etc.

On arrive ainsi aux représentants de la Flore montagnarde dont

1 Lecoq. Géogr. bot. de l'Europe, t. II, p. 139.

2 Un autre fait que l'on peut apporter à l'appui de ces obserrations, c'est que les plantes méridionales errat ques qui se sont fixees depuis quelques années dans les environs de Lyon, telles que Pterotheca nemausensis, Barkhausia setosa, etc. se sont étendues rapidement sur les coteaux du Rhòne et dans la direction du Montd'Or. mais nullement dans les cultures du Lyonnais granitique. (Voy. précédemment p. 35). 
la présence dans les Bas-Plateaux est une des particularitésłde leur régétation.

\author{
4. Flore monlaguarde : espéces dn plateau central \\ et de louest
}

Un certain nombre d'espèces de la région montagneuse qui domine les Bas-Plateaux du Lyonnais descendent jusque sur ces Bas-Plateaux et dans les vallées qui les séparent. En voici le tableau :

Ramunculus hederacens; habite les source:, les mares des monts du Lyonnais: les Halles, Aveize, Saint-Martin-en-Haut, Saint-Bonnet-le-Froid; descend a Vaugneray, Soucieu, et au commencement de la vallée du Garon;

Cardamine impatiens: deseend des monts qui entourent Sainte-Foy-l'Argenticre et Iscron dans les vallées de l'Iseron et du Ratier, à Beannant, Francheville, Tassin; des montagnes de Tarare, dans les vallées de la Turdine et de l'Azergue;

Polygala depressu: pl. olservée aux environs du Chatelard et de l'Orjol pri: l'. Irgentiere, de Larajasse, des Jumeaux; deseend à Charbonnières;

Dianthus deltoides : Fnvirons de l'Argentière, de Coise; descend à Charbonnieres, Tassin; ; avance jusque sous Limonest;

Spergula Morisonii: tous les sommets des environs de l'Argentière, de Saint-Bonnet-le-Froid; descent à Vaugneray et sur les boròs de la vallée du Garon, jusqüà Irrignais (c'est bien une plante montagnarde, car voỵ. LEGraxd, Stat. du Forez, p. 92);

Gcranizm nodosum: bois humides ct ruisseaux de toutes les montagnes, l'où il descend par les vallées, am bords de l'Azergue, a (rirors, ete.;

Lychnis silvestris: Coise; bords de l'Azergue;

Impiatiens noli-tanyere: Litrentiere; l'A bresle;

Rubus ,landulosus: Bois des monts du Lyomais, l'Argentière, Saint Bonnet ete.; arrive à Vaugneray, Tassin, Dardilly, ete.

Alchemilla vulgaris: de l'Argentière, Saint-Bonnet-le-Froid, descend à Chaponost;

Epilobium spicatum : environs de l'Argentière, Fenoyl; Tassin;

Montia rivularis : forme montagnarde du $M$. minor, qui des monts du Lyomnais, de l'Argentière, etc., descend à Soncieu, sur les Jor'ds du Garon, à Chäponost, à Tassin ;

Senecio adonidifolius: belle espèce caractéristique du plateau central, du Pilat et du Forez, qui existe aussi, mais rare, dans le Beaujolais et les monts du Lyon- 
nais (où elle a été trouvée près d'Iseron par MH. Guichard et Eug. Magnin), descend quelquefois jusqu’à Charbonnières et Tassin;

Gnaphalium silvaticum : Saint-Bonnet-le-Froid, l'Argentière; delà à Brignais, Tassin et même dans la Dombes (mais c'est bien une espèce montagnarde, car Legrand, Stct. du Forez, p. 154);

Crepis paludosa: de l'Argentière, de Saint-Bonnet-le-Froid, les Jumeaux et Iseron, descend dans la vallée du Garon; (cf. Legrand, op. cit., p. 164);

Jasione Carioni Bor.: Duerne, Iseron, Saint-Bonnet; Charbonnières, Chaponost, Beaunant, etc.

Lysimachia nemon'um: Commun dans les bois humides des montagnes, à l'Al'gentière, Iseron, ete.; descend à Vangneray, Mornant, Dardilly, etc.

Myosotis silvatica : Monts du Lyonnais, le Garon, Tassin;

M. Balbisiana Jord. : Saint-Laurent de Chamousset, Saint-Bonnet-le-Froil, Vaugneray;

Digitalis purpurea : pl. caractéristique des monts du Lyonnais (Saint-Bonnet, Iseron, Duerne, etc.); descend par l'Iseron, jusqu'all-dessus de la MaisonBlanche, et par le Garon, jusqu'au moulin du Barail;

Digitalis grandiflora: Le Fenoyl, Saint-Bomnet, Sourcieu, Soncien, le Garon;

Polygunum Bistorta: L'Argentière, Vaugneray, Soucieu, Francheville;

Carex pulicaris : LArgentière, lseron, Saint-Bonnet, Beaunant;

Deschampsia crespitosa : de la montagne, où il est commun, descend sur les bas-plateaux (cf. Legrand, op. cit., p. 238);

Les Aspixium aculeatum, Polystichum spinulosum, Cystopteris fragilis, A'plenium septentrionale, descendent aussi des monts du Lyomais, sur les has-plateaux et dans les vallées.

On pourrait y ajouter encore Parnassia palustiris, Elilobium obscurum, Menyanthes trifoliata, Pedicularis palustris, etc., et autres plantes des marais tourbeux, fréquentes dans la montagne, mais qui se retrouvent dans toutes les prairies marécageuses des environs de Lyon.

La plupart de ces espèces habitent les monts du Forez, du Pilat, et du Plateau central, qui paraît être leur centre de dis persion.

Quelques plantes de l'O. arrivent jusque dans nos contrées : par exemple, les Ulex europaus et nanus, qu'on suit jusque dans la Dombes, extrême limite de leur extension à l'E. de la France, et près de nous les Astrocarpus Clusii et sestmoitles dans Saône.. 
et-Loire, l'Adenocarpus complicatus dans la Loire et danș la fo rêt de Serres ${ }^{1}$, eic.

B. Comparaison de la végétation du Bas. Lyonnais avec les Flores voisines, non contrastantes.

La comparaison la plus instructive au point de vue de l'influence exercée par les différents sols sur la régétation est cerlainement celle de la Flore granitique du Lyonnais avec la végétat: on calcicole du Mont-d'Or et des coteaux du Rhône; mais derant exposc $?^{*}$ avec les mêmes déreloppements la Flore de ces régions et voular.t éviter les répétitions, nous renvoyons à ce moment leur ètude comparative, nous bornant aujourd'hui à indiquer les rapports que présente la Flore des bas -plateaux lyonnais avec les contrées ana logues comme sol et comme végétation, qu'elles soient situées dans son voisinage immédiat (alluviol.s de l'Azergue, cornes vertes du hassin de l'Arbresle, monts du Lyonnais), ou dans un rayon plus éloigné (plateau des Dombes, plaine clu Forez.)

\section{Alluvions de l'A zergue}

Elles recouvrent la partie septentrionale des bas-plateaux jusque sous Dardilly et près de la Tour -de-Salragny. Ces alluvions sont formées par un mélange de terre, de sable et de cailloux arrachés aux montagnes du Beaujolais et du Lyonnais par l'Azergue et ses affluents la Turdine et la Brevenne; sol essentiellement siliceux, comme le montrent les éléments qui le composent: quartz, diorite, amphibolite, granite, gneiss, porphyres, cornes vertes et calcaires (en petite quantité) ${ }^{2}$; aussi dans quelques courses que nous avons faites aux environs de la Tour-de-Salvagny et de Dommar-

1 Michalet. Hist. nat. Jura, t. II. Bot.p. 32 ; Cariot. É. des Fl. Ge éd. t. II, p. 153, etc.

On peut rapprocher de ces exemples l'Endymion nutans, plante de la régrion $\mathrm{N}-\mathrm{O}$. de la France, qui aurait été trourée entre Écully et Charbonnières; (Caniot, ibid, p. 713.)

? Voy. Falsan et Locard: Mont-d Or, p. 391. 
tin, avons-nous pu facilement constater que leur végétation ne diffère en rien de celle des alluvions alpines siliceuses de Charbonnières et Dardilly ${ }^{1}$.

\section{Cornes vertes}

Au N.-O. des bas-plateaux du Lyonnais, sur les limites de notre région, apparaît une formation spéciale quant à l'aspect des terrains qui la composert, sinon par sa régétation; ce sont les schistes qu'on observe à partir de Sourcieu, à Sain-Bel, à l'Arbresle, à Cliessy, etc., et qu'on appelle dans la contrée du nom de cornes vertes ou cornes rouges, suivant leur coloration ${ }^{2}$; riches on éléments silicatés, quelquefois fortement miracés, ils se rapprochent de nos gneiss par leur composition et leur mode de désagré . gation. Aussi la Flore qui recouvre leurs rochers ou les éboulis qui en proviennent, n'offre-t-elle aucune différence avec celles des gneiss du Lyonnais. On peut voir, en effet, abondamment sur les cornes vertes de l'Arbresle ou de Sain-Bel :

Sarothamnus vulgaris,

Erica vulgaris.

Andryala sinuata.

Malva moschata.

Jasione perennis.

Hieracium Yilosella.

Lampsana minima.

Linaria striata.
Hieracium umbellatum.

Scleranthus annuus.

Scl. peremnis.

Rumex Acetosella.

Thrincia hirta.

Chondrilla juncea.

Melica ciliata.

Papaver Argemone, etc.

Notons spécialement (non comme caractéristiques) le Glaucium luteım sur les cornes vertes, derrière la gare de Sain-Bel, le Melica ciliata, très commun dans tous les endroits bien exposés des environs de l'Arbresle.

Le Bouleau et le Châtaignier croissent avec rigueur sur ces schistes, tandis qu'ils ne viennent pas, ou végètent mal, sur le

1 L'ètude de ces alluvions se rattache à celle des monts du Lyounais et du Beaujolais, dont la Flore est loin d'ètre exactement connue, surtout pour ces derniers. Nous espérons pouvoir l'étudier plus tard.

${ }^{2}$ Voy. Founnet. Géologie lyon naise, (2e pagin.) p. 60. 
choin du $\mathrm{N}$. de l'Arbresle. Du reste, on observe un contraste frappant et tout a fait caractéristique entre la végétation descornes vertes et celle des terrains jurassiques qui s'ètendent de Bully i Châtillon d'Azergues. En sortant de l'Arbresle, dès qu'on passe des cornes vertes sur le sol calcaire (trias et surtout lias), on roit les plantes de l'énumération précédente disparaitre complètement et les suivantes apparaître : Berberis, Cerasus Mahaleb, Lien . trophyllum lanatum, Physalis Alkekengi, Lithospermum purpureo-camuleun, Melampyrum cristatum, etc.; puis, sur les placages des alluvions locales (siliceuses) de l'ancien glacier de la Brevenne, reviennent le Sarothamne, la Bruyère, les Châtaigniers, etc.

3. Monts du Lyonnais (et du Forez)

Les monts du Lyonnais, c'est-à-dire la chaîne qui s'ètend du Mercruy à Riverie, en passant par Saint-Bonnet, Iseron, Duerne, Sainte-Foy l'Argentière, Aveize, Saint-Hartin en Haut, Larajasse, Saint-André la Côte, etc., sont formés, comme on le sait, par des terrains identiques à ceux des Bas-Plateaux; c'est un sol exclusivement siliceux et à Flore éminemment silicicole; aussi en s'élevant des bas-plateaux sur les monts proprement dits du Lyonnais, n'obserre-t-on d'abord aucune différence dans la végétation, si ce n'est que les espèces montagnardes indiquées plus haut comme lescendant sur les bas-plateaux, deviennent plus communes; ce n'est qu'en arrivant à une certaine hauteur, en atteignant la zone des Pins et des Fayards (600 m. enriron), qu'on voit apparaitre les espèces nouvelles, dont la limite inférieure est sous l'influence de l'altitude; les principales de ces espèces, propres á la zone de la montagne, et qui ne descendent pas dans la région des basplateanx, sont :

Ranunculıs aconitifolius : Saint-Bonnet;

Cardamine amara: Saint-Bonnet;

Trifolium spadiceum : les Jumeaux, Saint-Bonnet, Duerne;

Rubus Idæus : Duerne, L’Argentière;

Circea intermedia: Saint-Bonnet; 
Sechm villosum : Saint-Bonnet, Iseron;

Ribes alpinum: Le Fenoyl, Saint-Laurent de Chamousset;

Conopodium denudatum: Larajasse, Pomeys;

Sambucus racemosa : L’Argentière, Duerne, Iseron, Saint-Bonnet le Froid;

Lonicera nigra : L'Argentière ;

Galium saxatile : Iseron, les Jumeanx, Saint-Bomet le Froid;

Senecio Fuchsii : Saint-Bomet, Duerne;

Centaurea nigra: Saint-Bonnet;

Vaccinium Myrtillus : toute la chaìne:

Pirola minor : Meys, Saint-André la Côte, Saint-Bomet;

Juncus supinus : Saint-Bonnet, les Jumeaux, Iseron ;

J. squarrosus: Saint-Bonnet, les Jumeaux;

Luzula nivea: Fenoyl, le Châtelard (l'Argentière), Saint-Bonnet;

Botrychium Lunaria: Saint-Bounet;

Polypodium Dryopteris: Haute-Rivoire, Duerne, Iseron;

Blechnum Spieant : Saint-André-la-Côte;

Equisetum silvaticum : Areize, Iseron, Saint-Bonnet.

On peut y ajouter Dentaria pinnata que nous avons trouvé dernièrement près du sommet du mont Arjoux.

De même que pour les eșèces montagnardes qui descendent dans la région des Bas-Plateaux, la plupart de celles-ci sont des plantes du Pilat et du Forez ${ }^{1}$.

Une autre remarque intéressante, c'est que quelques-unes de ces espèces descendent plus bas dans le Forez que dans le Lyonnais. par exemple: Ranunculus aconitifolius, Seclum villosum, Ribes alpinus, Sambucus racemosa (voy. Legrand, Stat. du Fore: pp. 66, 127, 129,140); cela tient à ce qu'à latitude égale, la vallèc, de la Loire est plus froide que celle du Rhône (voy. Legrand, Stat. ru Forez, pp. 29-30).

1 Pour la comparaison de la Flore des monts du Beaujolais et du Lyonnais arec relle de la chaine du Forez, voyez Legrand. Stat. bot. du Forez, p. 40 et 41 . 
$4^{\circ}$ Comparaison dela Flore des bas-plateaux avec colle de la plainedu Forez et de la Dombes à étangs

Si l'on compare la végétation des terrains granitiques des basplateaux du Lyonnais arec celle des sables siliceux de la plaine du Forez et des sols argilo-siliceux de la Dombes à étangs, on constate la plus grande analogie, qui se révèle, en attendant une étude plus complète, par la simple énumération qui suit ${ }^{1}$ :

* Myosurus minimus.

* Ranunculus hederaceus.

Teesdalia nudicaulis.

Dimnthus de'toides.

Sllene Armeria.

Lychnis Viscaria.

Spergula Morisonii.

* Alsine segetalis.

* Hypericum pulchrum.

* Ulex curoprens.

* U. namis.

* Sarothamnus vulgaris.

* Trifolium striatum.

T. subterraneum.

* T. elegans.

* Lathyrus Nissolia.

${ }^{*}$ L. angulatus

^L. hirsutus.

Potentilla micrantha.

Epilobirum collinum.

E. lanceolatum.

E. obscurum.

* Lythrum Hyssopifolia. Herniaria glabia.

* Montia minor.

$M$. rivularis.

Sedum elegans.
Umbilicus pendulinus.

Pencedanum parisiense.

* Enanthe Phellandrium.

Rupleurum Jacquinianum.

* Gnaphalinm silvaticum.

* Filago montana.

Senccio adonidifolizs.

* S. erraticus.

* Arnoseris minima.

Jasio perennis.

+ Centunculus minimus.

Myosotis luten.

Digitalis purpurea.

Veronica verna.

* Littorella lacustris.

* Rumex maritimus.

* Salix cinerea.

Orchis coriophora.

Lizula maxima.

Juncus capitatus.

J. pygmæus,

J. hybridus.

* Scirpus setacens.

* Sc. supinus.

Carex pulicaris.

* Alopecurus fulvus.

Aira aggregata.

${ }^{1}$ Les plantes dont le nom n'est pas suivi d'un astérisque (") sont celles communes aux bas-plateaux Iyonnais et à la plaine du Forez; lastérisque indique que la plante se trouve, en outre, dans la Dombes à èlangs. Les espèces soulignées sont des plantes communes aux montagnes ou aux rochers du Lyonnais et du Forez, et on doit les sup. primer dans la comparaison avec la Dombes et la plaine du Forez. 
Avena tenuis.

Danthonia decumbens.

* Festuca heterophylla.

* Nardurus tenellus.

Nardus stricta.
Ophioglossum vulgatum.

Asplenium Halleri.

A. septentrionale.

* Pteris aquilina.

Du reste, lorsque nous aurons donné le tableau de la végétation de la Dombes, il nous sera plus facile de faire ressortir les analogies et les différences qui existent entre les végétations de ces régrions, et de discuter quelle est la part qui revient aux influences chimiques et aux influences physiques dans l'analogie si grande que présente leur tapis végétal. 



\section{DEUXIÈME PARTIE}

\section{COTEAUXET VALLEES DU RHONE ET DE LA SAONE}

Sous la dénomination de Coleaux du Rhône et de la Saône, nous comprenons :

$1^{\circ}$ Les coteaux qui s’étendent sur la rive gauche de la Saône et la rive droite du Rhône, au-dessus de Lyon, c'est-à-dire les bords occidentaux et méridionaux du plateau de la Dombes, de Trévoux à Lyon, et de Lyon à Meximieux;

$2^{\circ}$ Les Balmes-Viennoises, situées sur la rive gauche du Rhône, de Jons à Villeurbanne et de Saint-Fons à Saint-Symphorien d'Ozon, en y joignant les coteaux du Bas-Dauphiné qui les avoisinent;

$3^{\circ}$ Les coteaux situés sur la rive droite du Rhône, de Givors à Lyon, c'est-á-dire les plateaux de Sainte-Foy, Saint-Genis-Laval, Irigny, Charly et Millery.

A leurs pieds s'étendent les alluvions de la Saône et les allu-vions du Rlione, ces dernières reprèsentées par des îles nombreuses, souvent fort étendues, et par des plaines qui prennent leur plus grande extension vers le confluent du Rhône et de l'Ain, où elles donnent naissance à la Valbonne.

Toutes ces localités présentent une analogie remarquable au double point de vue de leur constitution géologique et de leur végétation, ainsi que les détails que nous allons donner sur la Topographie, la Géologie et la Flore de chacune d'elles, vont le déinontrer. 


\section{SEGTION PREMIĖRE}

\section{COTIÈRE OCCIDENTALE ET IMÉRIDIONALE DE LA DOMBES}

Les hauteurs qui s'èlèvent de Trévoux à Lyon et de Lyon à Meximieux ne sont, à proprement parler, que les bords du plateau de la Dombes; mais, bien que leur constitution géologique soit celle du plateau bressan, l'aspect et la nature des terrains qui les composent et la végétation qui les recouvre en diffèrent complètement : nous allons en voir les raisons.

\section{GHAPITRE PREMIER}

\section{APERSGU TOPOGRAPHIQUE}

La còtière dont il s'agit est formée par une série de mamelons qui fontsuite au plateau de la Dombes et qui sont séparés entre eux par des dépressions plus ou moins profondes, donnant passage soit a des rivières alimentées par les eaux de pluie et l'écoulement réglé des étangs, soit à des torrents qui ne se forment qu'au moment des orages.

Son élévation au-dessus de la plaine alluviale du Rhône et de la Saône est de 100 à $150 \mathrm{~m}$. en moyenne; elle parait aller en augmentant à mesure qu'on se rapproche de Ljon; mais en réalité, le plateau conserve dans son ensemble une altitude noyenne à peu près constante, ainsi qu'on peut s'en assurer en comparant la cote $280 \mathrm{~m}$. au-dessus de Trévoux et celle de $280 \mathrm{~m}$. aux Mercières, près de Rillieux; cependant, dans sa partie méridionale, la còtière se relève légèrement au-dessus de Beynost, à Margnolas, où elle atteint son altitude maximum $(329 \mathrm{~m}$.), et s'abaisse ensuite lentement vers Meximieux. Si cette falaise s'accentue darantage dans 
les environs immédiats de Lyon, c'est que les vallées du Rhòne et de la Saône s'abaissent graduellement et que ses flancs deviennent de plus en plus abrupts.

Elle se divise naturellement en deux parties : une occidentale, dirigée du N. au S.., au pied de laquelle coule la Saône; l'autre méridionale, dirigée du N.-E. au S.-O, limitant au N. les alluvions du Rhône et la plaine de la Valbonne.

La première, dont nous n'étudions en ce moment que les parties situées entre Trévoux et Lyon, est coupée par de nombreux vallons dirigés du N. E. au S.-O., donnant pour la plupart passage à des ruisseaux de peu d'importance, mais dont les flancs ordinairement boisés sont les stations préférées par quelques-unes des espèces les plus intéressantes de la Flore. Les principaux de ces ruisseaux sont :

1. 2. Les ruisseaux de Reyrieux et de Parcieux;

3. Le ruisseau du Grand-Rieux, alimenté par les étangs de Saint-André de Corcy ; il traverse les communes de Civrieux et de Massieux;

4.5. Les ruisseaux de Genay et de Neuville;

6. Le ruisseau des Échets ou de Rochetaillée; c'est le plus im portant de la région; il sert d'écoulement au bassin des Échets dont la surface de 4,800 hect., reçoit annuellement 4,800,000 mètrey cubes d'eau'.

7. Le vallon de Sathonay, le plus intéressant au point de vue botanique; il est formé à l'origine par plusieurs dépressions qui prennent naissance sous les villages de Vancia, Sathonay et Rillieux, se réunissent et forment le vallon proprement dit ; dans le fond coulele ruisseau de Ronzières, qui se jette dans le Rhône sous Fontaine; les bords de ce vallon sont garnis de bois ou de pelouses incultes, riches en espèces caractéristiques; dans sa moitié inférieure, la vallèe s'élargit et permet l'établissement de prairies et de quelques autres cultures.

8. En deçà du vallon de Sathonay, le coteau est découpé par des

1 Voy. A. Magnin. Études géologiques, botaniques et statistiques sur l'impaludisme dans la Dombes, etc., 1876, p. 14. 
échancrures moins considérables, généralement derenues inaccessibles par des clôtures; ce n'est que dans quelques points rares, comme les pelouses et les bois du Vernay, les poudingues du vallon de Caluire et de la montée de la Caille, que le botaniste peut observer des traces d'une végétation spontanée.

Ces vallons séparent les plateaux de Genay, Montanay, Caillouxsur-Fontaine, Saint-Martin de Fontaine, Sathonay et de la CroixRousse.

La côtière méridionale présente une configuration analogue; en se dirigeant de Meximieux vers Lyon, on trouve successivement les vallons suivants, dirigés en général du N.-N.-O. au S.-S.--E. :

1. L'échancrure qui donne passage au Longerant, ruisseau qui sert à l'ècoulement des étangs de Saint-Éloi et des communes voisines; après avoir traversé Heximieux, ce ruisseau se perd dans les graviers de la Valbonne, près du domaine de la Rouge.

2. Après les hauteurs de Pérouges, du Bourg-Saint-Christophe, et de Béligneux, s'ouvrent les vallons du Cotey et de son affluent le ruisseau de Belignieux; le premier naît sous Faramans, passe entre les plateaux de Bressoles et de Pizay; le second sépare les hauteurs de Bressoles et de Bélignieux; ils se réunissent sous Dagnieux pour aller se jeter dans le Rhône, après avoir parcouru 2 kilomètres dans la plaine alluviale de Balan.

Ces vallées et les hauteurs qui les entourent offrent peu d'intérêt ; à part quelques endroits boisés, de peu d'étendue, leur's flancs, doucement inclinés, sont généralement garnis de cultures, principalement de rignes.

3. La Sereine, qu'on trouve ensuite, est la rivière la plus impor tante de la région; c'est aussi la vallée la plus intéressante pour le botaniste. Elle prend naissance par la réunion des biefs des nombreux ètangs de Birieux, du Monteillier, de Cordieux, etc., se dirige vers le S., sort du plateau à Montluel et de là s'étend pendant 6 kilometres environ dans la plaine alluviale de la Boisse et de Beynost, avant de se jeter dans le Rlòne entre Thil et Miribel. Audessous de Montluel, ses bords assez abrupts sont souvent garnis de bois et le fond de la vallée présente sur plusienrs kilométres de 
longueur, des prairies dont les parties marécageuses (marais de Sainte-Croix) renferment un grand nombre de plantes remarquables ${ }^{3}$.

De la Sereine à Lyon, la côtière est une falaise de plus en plus abrupte, aux flancs déchirés par une série de pittoresques échancrures, boisées, dont les principales sont : le torrent de Saint-Martin, le vallon de la route de Montluel à Trévoux, au-dessus de la Boisse, les vallons de la Miandière, de Saint-Germain, de Beynost, de Saint-Maurice, de Sermenaz, de la Cadette, de la Pape, de Crépieu et de Vassieux; ces derniers, autrefois riches stations classiques pour les botanistes lyonnais, deriennent de jour en jour plus inaccessibles par l'établissement de trop nombreuses clôtures.

Enfin, signalons à la base de la côtière une terrasse alluviale, très nette, déjà mentionnée, mais inexactement par Fournet ${ }^{2}$ et qui, par sa composition minéralogique et sa Flore, se rattache à la côtière qui la domine; cette terrasse s'étend, non pas de Beynost jusqu'à la Pape, mais de Neyron-d'en-Bas au delà de Balan, où elle se perd sur le bord de la Valbonne. Du. reste, elle n'est pas continue, mais se compose de trois tronçons ainsi disposés : le premier se détache de la côtière sous Neyron-d'en-Bas et s'étend jusqu'à l'extrémitè orientale de Miribel; le second naît insensiblement entre Saint-Martin et Saint-Maurice de Beynost, court parallèlement à la còtière en se perdant contre elle, au hameau du Péchu (commune du Beynost); le troisième commence vers l'église de la Boisse, se dirige vers le moulin Cassal et Balan; à partir de ce village, il suit le bord de l'ancien lit du Rhône et le plateau dont il est la limite méridionale, se continue avec la plaine de Valbonne. Ces balmes et ces plateaux qui leur succèdent sont élevés de 6 à 10 miètres au-dessus de la plaine alluvienne plus récente ${ }^{3}$.

1 Voy. Flore des Tourbieres et des marais tourbeux.

2 ".... Enfin je mentiounais les ébauches de nos grrandes vallées d'érosion arec eurs divers étages... Autour de Lyon, en purticulier, ces étages sont dessinés fort nettement, par exemple, à Villeurbanne le long de la Balme.Viennoise, à Beynost, d'où un gradin s'étend jusqu'à la Pape, etc... " Focrset. Gcol. lyonn., p. 299.

3 Dans un travail speccial, nous montrerons quelle a été l'intlueuce des cônes de déjections, qui débouchaient par les échancrures du coteau, sur la formation de ces terrasses. 
On verra, plus tard, qu'on observe une disposition analogue de l'autre côte du Rhône et que la Balme-Viennoise de Jonage à Villeurbanne correspond exactement à la terrasse que nous venons de décrire.

\section{GHAPITRE II}

GEOLOGIE ET NATURE DU SOL

Les travaux de MM. Em. Benoît, Falsan, etc. ${ }^{1}$ ont montré que le plateau bressan est constitue en allant de haut en bas par :

$5^{\circ}$ Le lehm ou terre à pisé;

$4^{\circ}$ La boue glaciaire ;

$3^{\circ}$ Les alluvions glaciaires ou conglomérat bressan;

$2^{\circ}$ Les sables et marnes pliocènes et mio-pliocènes;

$1^{\circ}$ La mollasse marine.

Or, les coteaux qui font le sujet de cette étude, n'étant que les bords du plateau bressan, ont exactement la même constitution : avec cette différence que le sol végétal, au lieu d'être formé comme dans le plateau bressan, par une ou deux seulement des couches supérieures, est ici formé aux dépens de toutes les assises qui s'ètagent en une coupe géologique naturelle.

Le lehm forme généralement les sommets de la côtière et les mamelons voisins situés en dehors de la zone à étangs; son épaisseur est variable, quelquefois considérable, comme à Vancia, au Haut-Neyron; on en trouve aussi des dépôts remaniés au pied de la còtière, dans le vallon de Fontaine, et de Miribel à Montluel sur

1 E. Benort. Esquisse géologique et agronomique de la Bresse (Bull. Soc. géoloy. de Fr., $2^{\circ}$ sèr., t. XV, 1858, p. 315 ; - FALSAN. Sur les anciens glaciers de la partie moy. du Rhône (Assoc. franç. pour l'avanc. des sc., session de Lyon, 1873; Falsan et Locand. Formations tertiaires et quaternaires des environs de Miribel (Ann.Soc. d'Agric. de Lyon, 1878), etc. 
la terrasse décrite plus haut, supportant les villages de Saint-Maurice, Beynost et la Boisse.

Il devient un sol meuble, perméable, silicéo-calcaire, ordinairement assez, riche en chaux; les analyses de M. Pouriau montrent, en effet, qu'il en contient normalement de 3 à $100 / 0^{1}$; on peut y trouver des kupfsteins ou concrétions calcaires, par exemple à Cordieux. Dans certaines parties cependant, surtont dans les points où le lehm repose directement en couches minces sur les alluvions glaciaires, ou à la partie supérieure des dépôts, on trouve un lehm devenu exclusivement siliceux par suite de l'entraînement du carbonate de chaux dans les couches profondes sous l'influence des infiltrations pluviales ${ }^{2}$. Les analyses de M. Pouriau ainsi que les nôtres lui donnent alors la composition moyenne suivante : silice, 74 à $80 \mathrm{p}, 0 / 0$; chaux. 0,50 à $1 \mathrm{p} .0 / 0$, jamais audessus.

Il importe d'avoir présente à l'esprit cette modification, si l'on veut interpréter sainement la présence de certaines espèces végétales.

Au-dessous du lehm se trouve la boue glaciaire; mais cette couche si répandue sur toute la surface du plateau bressan qui lui doit l'imperméabilité du sol et la possibilité de l'existence des ètangs, ce dépôt si important dans l'étude phytostatique de la Dombes, manque complètement sur le bord du plateau; recouverte resque partout par le lehm, elle n'occupe une surface de quelque étendue que dans la partie comprise entre Rillieux et la CroixRousse, et encore n'y contribue-t-elle à former que des terres cultivées; partout ailleurs la boue glaciaire n'apparait qu'en petits

1 Ex. : Lehm de Sathonay (village) : silice 74,24; chaux 5,76

$$
\begin{array}{ll}
\text { - } \quad \text { (sous-sul) : } & -72,14 ;-8,10 \\
\text { - du vallon de Fontaine : } & -80,12 ;-5,70 \\
- \text { de Cordieux : } & -80,35 ;-2,05
\end{array}
$$

Voy. Pouriau. Etudes géologiques, chimiques et agronomiques des sols de la Bresse et de la Dombes. (Ann. Soc. llagricul. Lyon, $3^{\circ}$ sèr., t. II, 1858, p. 184 et seq.)

2 Ce lehm spécial paraît correspondre, en partie du moins, au diluvium rouge de M. Sauvaneau (Ann. Soc. d'Agric. Lyon, 1845. Recherches sur la composition des terres végétales.) 
placages; elle n'a donc aucune influence sur la végétation; aussi ne nous $y$ arrêterons-nous pas davantage.

Il n'en est pas de même des alluvions glaciaires: leurs immenses amas de cailloux roulés, - quartzites, dioritines, pliyllades, calćaires jurassiques et autres roches des Alpes de la Savoie et du Bugey , - apparaissent dans toute la falaise, soit en place, soit sous forme d'éboulis recouvrant l'affleurement des couches plus inférieures. Les alluvions glaciaires, - conglomérat bressan d'Élie de Beaumont, - se présentent sous deux états bien diffẻrents : tantôt ce sont des amas de cailloux roulés, libres ou à peine liès entre eux par un sable fin, micacé et calcaire, passant quelquefois à de véritables bancs de sables et graviers; ailleurs ces masses de cailloux se sont solidifiées en poudingues parfois très résistants, par l'adjonction d'un ciment calcaire; on peut observer ces poudingues en beaucoup de points de la falaise, sous Sathonay, aux montées de Caluire, Cuire, de la Caille, sous la tour de la Belle-Allemande, a Saint-Clair, Crépieux, la Pape, le Mas-Rilliet, Beynost, etc.

Le sol formé par le conglomérat varie suivant sa nature; les poudingues se dissociant avec lenteur, forment ordinairement des pentes abruptes, recélant dans leurs excavations ou dans les fentes de leur ciment calcaire quelques cryptogames, Hépatiques, Mousses ou Lichens tout à fait caractéristiques. Les alluvions non solidifiées, cailloux, graviers, sables, constituent un sol aride, dont les parties supérieures peu inclinées sont garnies de pelouses, de broussailles ou de bois et les inférieures forment des pentes graveleuses, meubles, perméables, très arides; mais à la base de la falaise, les éboulis de ces alluvions, par leur mélange avec le lelim, donnent naissance à un sol meuble, très fertile, riche en chaux et en potasse $^{1}$, qui convient spécialement à la rigne, à la luzerne et aux cultures maraîchères qui en occupent du reste la plus grande ètendue.

ll est inutile de montrer par des analyses la richesse en chaux

${ }^{1}$ La chaux provient du Jehm, du ciment calcaire et de la décomposition des roches calcaires; la potasse, de la décomposition des ronches feldspalhiques. 
des poudingues à ciment calcaire; quant aux autres alluvions anciennes, les analyses de M. Pouriau montrent que la quantite de carbonate de chaux varie de 1 à 10 0/0, et qu'elle peut atteindre de 17 à 20 0/0 (carrières de sables de Sainte-Croix à Montluel) ${ }^{1}$.

Les sables et marnes pliocènes (de même que la mollasse marine) affleurent dans un grand nombre de points, comme à Tré voux, au Vernay, à Miribel, etc.; mais ou ces terrains ne se montrent que sous une faible épaisseur, ou bien ils sont recouverts par les éboulis du conglomérat; aussi leur influence peut-elle être considérée comme nulle sur la végétation de cette partie des coteaux du Rhône. Il en est de même des sables ferrugineux de Trévoux et des calcaires d'eau douce de Meximieux qui appartiennent au même ètage. Cependant il convient d'insister davantage sur l'horizon des marnes des Boulaies, près Miribel ${ }^{2}$, situées à l'altitude de 225 à $245 \mathrm{~m}$., soit à environ $70 \mathrm{~m}$. au-dessus de la plaine alluviale. Ainsi que Fournet l'avait déjà soupçonné ${ }^{3}$ et ainsi que nous l'avons vérifié récemment, c'est cette couche imperméable qui règle le régime des sources le long de la falaise méridionale depuis Neyron jusqu'à Montluel4 ${ }^{4}$ et par suite détermine l'apparition, tout a fait localisée à ce niveau, de plusieurs plantes hygrophiles.

Un niveau semblable de sources, déterminé, du reste, par des accidents géologiques de même ordre, se retrouve le long de la côtière occidentale de la Dombes; c'est à lui que se rattachent les sources de Reyrieux, Parcieux, Genay, la fontaine Camille (de Neuville), etc. ${ }^{5}$.

En résumé, des détails qui précèdent, nous pouvons conclure

1 Diluvium à gros élément : Grand-Peuplier. . = Silice 85,30; chaux $=3,18$

\begin{tabular}{|c|c|c|c|c|c|c|c|}
\hline- & - & Avant Miribel. . & $=$ & - & 74,$52 ;$ & - & $=8,80$ \\
\hline - & - & Sortie de Miribel. & $=$ & - & 77,08 & - & $=4,96$ \\
\hline 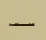 & - & Château de la Pape & $=$ & - & 76,46 & - & $=6,42$ \\
\hline - & - & Saint-Clair & $=$ & - & 81,38 & - & $=6,50$ \\
\hline
\end{tabular}

Pouriav. Op. et loc. cit., p. 203.

2 Voy. Falsan et Locard. Note sur les formations tertiaires et quat. cles environs de Miribel. (Ann. Soc. Agric. Lyon, 19 juillet 1878.)

3 Fournet. Mémuires sur les soulces (Ann. Soc. Algric. Lyon, 1839, t. II, p. $210-212$.

4 Principalement dans les communes de Mirihel, Saint-Maurice et Beynost.

s Voy. Fournet. ibid., p. 201. 
que les sols qui dominent dans les coteaux du Rhône et de la Saône sont des terrains calcaires; et, comme M. Pourian l'avait constaté pour les terres arables ${ }^{1}$, nous pouvons donner comme loi générale que le sol devient de plus en plus riche en carbonate de chaux à mesure qu'on s'èloigne des terrains à étangs de la Dombes, et à mesure qu'on descend du plateau, vers le Rhône ou la Saône. Nous verrons dans un instant l'application de ces faits à la régétation de la région.

\section{GHAPITRE III}

VÉGÉTATION

§ I. - Enumération des plantes d'aprés les localités

La végétation des côtières occidentale et méridionale de la Dombes, bien qu'uniforme dans son ensemble, présente cependant assez de particularités liées surtout aux accidents topographiques, pour que des exemples d'herborisation soient nécéssaires.

\section{A. Herborisation dans le valion de Fontainfes a Sathonay et a Rillieus ?}

En remontant le ruisseau de Ronzière, ou en suivant la route de Fontaine au camp de Sathonay, on rencontre d'abord, après avoir dépasséles propriétés closes, des éboulis provenant des sables mollassiques et du conglomérat bressan, des amas de lehm à Succinea oblonga, Helix rupestris, etc., recouverts de bois ou de broussailles à Robinia pseudo-acacia, Cratrgus oxyacantha, Corylus Avellana, etc., et ne renfermant, à part les Coronilla Emerus, Saponaria officinalis, Mercurialis perennis, etc., que des plan-

1 Pouriau. Op. cit.. p. 184 et seq., p. 203.

2 Voy. Ann. Soc. bot. de Lyon, t. III, P. 79, 81, 87; - t. IV, p. 159 ; - t. V, p. 126 , etc. 
tes triviales. Mais plus haut, vers la séparation des chemins de Sathonay, du Camp et de Rillieux, le vallon se resserre, se divise en plusieurs vallonnets étroits, garnis de bois frais qui renferment un grand nombre de plantes intéressantes:
Anemone nemorosa.
Polygonatum anceps.
Primula grandiflora,
P. multiflorum.
Viola scotophylla.
V. Reichenbachiana.
Convallaria majalis.
V. Riviniana.
Luzula campestris.
Orobus tuberosus.
L. Forsteri.
Pulmonaria tuberosa,
L. vernalis.
Veronica serpyllifolia.
Carex silvatica.
Phyteuma spicatum.
Melica uniflora.
Ranunculus nemorosus.
Hypnum triquetrum.
Euphorbia amygdaloides,
H. cupressiforme.
H. splendens.
E. dulcis.
Atrichum undulatum, etc.

Notons plus spécialement, comme plus rares :
Sanicula europæa.
Maianthemum bifolium,
Asperula odorata,
Veronica montana.

Paris quadrifolia

et quelques pieds disséminés de Cormus mas.

Sur le bord des chemins, dans les éboulis découverts :

Fumaria parviflora.

Sedum Fabaria.

S. Telephium.

Erucastrum obtusangulum.

Stellaria Holostea.
Cerastium brachypetalum.

Saxitraga granulata.

S. tridactylites.

Phleum asperum.

Bromus madritensis.

et dans les lieux plus ombragés: Taraxacum murinerve, Lamium incisum, Mcehringia trinervia.

En arrivant au sommet des vallons, les éboulis du conglomérat funt place aux alluvions glaciaires ou au lehm, mais à ce lehm devenu presque exclusivement siliceux que nous avons signalé dans les considérations géologiques; on roit alors disparaitre le Coronilla Emerus, dominer les Quercus sessiliflora, Q. pubescens, les Luzules, les Dicranum, et apparaître le Sarothamne et la Bruyère. 
Si l'on avance plus loin sur les terres du plateau, dans la direc. tion de Rillieux ou du Camp, on observe, sur la terre argilo-siliceuse, les plantes caractéristiques de la Dombes, comme : Sarothamnus vulgaris, Spergula arvensis, S. rubra, Ranunculus philonotis, les Filago, Sagina patula, Myosurus minimus (vers la Gare), Montia minor (Boullu, Cusin), etc.

Les cultures, les moissons donnent aussi quelques plantes intéressantes, mais non caractéristiques, telles que Caucalis daucoïdes, Bunias Erucago, Pterotheca nemausensis, Barkausia setosa, Tragopogon major, Veronica triphyllos, Neslia paniculata (rare), etc.

Nous avons suivi jusqu'à présent le versant tourné au $N$.; si nous passons sur le flanc du vallon, exposé au midi, nous trouverons une régétation bien différente; au lieu de bois frais, ce sont des pelouses arides, reposant sur les cailloux du conglomérat, traversées dans maints endroits par des masses de poudingues, à végètation spéciale.

Ces pelouses supportent en effet, au milieu des Festuca glauca, Thymus Serpyllun, Teucrium Chamxdrys, Potentilla verna. Helianthemum procumbens, etc. :

Pulsatilla r'ubr'a.

Geranium sanguineum.

Potentilla rupestris.

Anthyllis Vulneraria.

Hippocrepis comosa.

Orehis Simia.

0. fusca.

Raumeulus Chœrophyllos.

Trifolium alpestre.

Galimm tricorne.
Convolvulus cantabrica.

Helianthemum denticulatum.

Veronica prostrata.

Orobanche Epithymum.

Carex præcox.

C. muricata.

C. divulsa (rare).

Barbula squarrosa.

B. inclinata.

Les parties supérieures plus sableuses donnent :

Spergularia rubra.

Seleranthus annuus.

Myosotis ver'sicolor.
Veronica præcox.

Vicia lathyroides, etc.

Sur le ciment calcaire des poudingues: Barbula membranifolia. 
B. Excursion de la Pape a Rillieux par le valion de ha Cadettel

En sortant de Lyon par Saint-Clair, on passe devant les vallons de Vassieux, Crépieux, autrefois localités classiques pour les botanistes lyonnais, mais aujourd'hui défrichées ou inaccessibles. Cependant, sur les poudingues qui bordent la route vers Crépieux, on peut observer les espèces manifestement calcicoles suivantes :

Barbula membranifolia.

Thalloidima vesiculare.

Psoroma fulgens.

Le vallon de la Cadette, auquel on arrive bientôt, lorsqu'on a dépassé la Pape, est une échancrure profonde, creusée dans les alluvions glaciaires, qui naît par trois branches sous le village de Ril lieux et vient aboutir au Rhône. Il présente à explorer des poudingues arides, des pelouses sèches, des broussailles et des bois.

T'out d'abord, sur les blocs de poudingues qui ont roule de plus haut, on remarque le Barbula membranifolia, espèce méridionale qui se maintient là, gràce à l'exposition et à la nature calcaire du sol, et les Hutchinsia petrcea, Alsine Jacquini, Helianthemum canum, plantes originaires du Jura, mais des stations chaudes, bien exposées au midi, et qui, descendues du Bugey par le Rhône, se sont acclimatées dans nos environs et y croissent spontanément.

Sur les pelouses qui garnissent les flancs et le fond du vallon, on observe :

Carex humilis.

Orchis anthropophor'a.

Ophrys apifera.

Scilla bifolia.

Hutchinsia petræa.

Helianthemum procumbens.

H. denticulatum.
Barbula squar'rosa.

Hypnum rugosum.

Thuidium abietinum.

Cladonia furcata.

Cl. squammosa.

Psora decipiens, etc.

4 Voy. Ann. Soc. bot. de Lyon, t. II, p. 44; - t. V, p. 170, 173, etc. 
Et un perı en dehors des pelouses, principalement sur les éboulis : les Erucastrum, Diplotaxis, et le Dianthus Scheuchzeri Rchb. (D. silvestris Wulf. var. collivagus Jord.), à dispersion fort intéressante, comme nous le verrons dans un instant.

Le versant qui regarde l'O. a un sol plus frais, recouvert par : Barbula inclinata, Fissidens adiantoides, IVeissia viridula, Cylindrothecium Montagnei, Hypnumcupressiforme, Funaria calcarea (rare), Phascum bryoides, etc. Dans les bois qui le garnissent et s'étendent jusqu'au sommet du vallon :

Atrichum undulatum.

Des Hypnum divers.

Thuidium tamaricinum.

Les Luzules et Primevères halituelles.

Pulmonaria tuberosa.

Orobus niger.

Arenaria trinervia.
Polygonatum anceps.

Melittis Melissophyllum.

Phyteuma spicatum.

Melampyrum vulgatum.

Orchis bifolia.

O. ustulata.

Coronilla Emerus, etc.

G'est non loin de là, dans les bois situés derrière le chàteau de M. Réveil, que se trouvent le Centaurea lugdunensis, variété du C. montana, et une des rares localités de l'O. papilionacea.

En continuant de remonter le vallon, on récolte une série d'espèces des coteaux secs, préférant cependant les sols calcaires; ce sont: Helianthemum procumbens, H.pulverulentum, Geranium sanguineum, Trifolium alpestre, T. montanum, Convolvulus cantabrica, Veronica spicata, Orchis variegata, 0. fusca, etc.

En arrivant vers le sommet du vallon, on trouve dans les bois quelques pieds de Cornus mas; notgons aussi l'Alsine viscosa, le Valerianella microcarpa, dans les pelouses du fond du vallon, et au sommet, au-dessus de Crépieux, le Bromus maximus dẻjà indiqué par J. Fourreau et retrouvé par M. Cusin.

Mais ce cont les coteaux qui s'étendent de li ver's Neyron et Miribel qui offrent le tableau le plus complet de la Flore dite de la Pape. 
C. Herborisation dans le cothau de Neyron 1

Si, à partir du pont de la Cadette, on continue de suivre la route nationale, on arrive à des promontoires d'alluvions glaciaires dont les flancs forment de grandes masses d'éboulis, exploités comme gravières, sur la gauche de la route.

Nous croyons devoir donner une liste un peu ètendue des plantes qui y croissent en commençant par les plus communes; on aura ainsi une idée du tapis végétal de cette station si caractéristique des coteaux du Rhône.

Dans les gravières mêmes :

Thymus Serpyllum.

Setaria glauca.

Ononis Natrix.

Artemisia campestris.

Asperula cynanchica.

Teucrium Chamædrys.

Setaria viridis.

Hordeum murinum.

Festuca glauca.

Gypsophila 'saxifraga.

Hypericum perforatum.

* Teucrium montanum.

Alyssum calycinum.

Euphorbia Cyparissias.

Stachys recta.

Brachypodium silvaticum.

Centaurea paniculata.

* Convolvulus cantabrica.

Chondrilla juncea.

Plantago arenaria.

Galium lieve.

* Silene conica.

Dianthus prolifer.

*D. Schcuchzeri.
Helianthemum vulgare.

- Helichrysum Stœehas.

Sedum album.

I'opulus Tremula.

Quercus Robur.

Corylus Avellana.

Vincetoxicum officinale.

Scrofularia canina.

Silene nutans.

* Cerasus Mahaleb.

Echium vulgare.

Scabiosa Columbaria.

* Geranium sanguineum.

Origanum vulgare.

- Buxus sempervirens.

* Thesium divaricatum.

Viburnum Lantana.

Juniperus communis.

Sedum refiexum.

Campanula persicifolia.

* Coronilla minima.

Calamintha Acinos.

C. Nepeta.

*Globularia rulgaris.

Les pelouses qui s'ètendent au-dessus, jusqu'au sommet du coteau et les broussailles formées de Quercus sessiliflora, Q. pu . bescens, Cerasus Mahaleb, Ligustrum vulgare, Prunus spinosa, Coronilla Emerus, Ruscus aculealus, Buxus sempervi-

1 Voy. Ann. Soc. bot. Lyon, t. IV, 1. 151. 
rens, renferment en outre des espèces vulgaires : Asperula cynanchica, Sechum reflexum, Centaurea paniculata, Artemisia campestris, Thymus Serpyllum, Seclum album, S. sexangulare, S. anopetalum, Calamintha Acinos, Eryngium campestre, Festuca glauca, Linum catharticum, Allium sphierocephalum, Poiygala vuigaris, Helianthemum procumbens, $H$. pulverulentum, Geranium columbinum, $G$. dissectum, $G$. molle, G. pyrenaicum, Bromus mollis, les espèces plus spéciales qui suivent:

Trifolium procumbens.

T. glomeratum.

Hypochæris maculata.

Silene untans.

Vincetoxicunı officinale.

Helianthemum obscurum.

H. guttatum.

Bromus crestus.

Galium lieve.

G. decolorans.

Thesium divaricatum.

Orchis pyramidalis.

Trifolium alpestre.

T. montanum.

Galium corrudæfolium.

Bupleurum aristatum.

Potentilla rupestris.

Trinia vulgaris.

Globularia rulgaris.

Orchis Simia.

ก. Morio.

Aceras anthropophora.
Carex nitida.

Geranium sanguineum.

Peucedanum Cervaria.

Rubia peregrina.

Ophrys Arachinites.

Lithospermum arvense.

L. purpureo-cxruleum.

Convolvulus cautabrica.

Linum gallicum.

Ononis Columnre.

Veronica spicata.

Hippocrepis comosa.

Veronica prostrata.

Coronilla minima.

Helianthemum denticulatum.

Teucrium montanum.

Carex Halleriana.

Dianthus Scheuchzeri.

Orchis fusca.

0 . variegata.

Pulsatilla rubra.

Limodorum abortivum.

et le rarissime Cistus salvizfolizus, plante du Midi, remontant jus-qu'ici, au lieu dit Montgoîtron, mais qui disparaîtra bientôt par suite des défrichements.

Dans les parties supérieures, sur le lehm devenu siliceux :

Genista pilosa.

G. germanica.

G. sagittalis.

Trifolium arvense.

Potentilla argentea.
Festuca heterophylla

Aira caryophyllea.

Jasione montana.

Anarrhinum bellidifolium.

Sarothamnus vulgaris. 
Les cultures, les terrains meubles du lehm situé sur le plateau donnent : Caucalis daucoides, Iberis pinnata, Calamintha Acinos, Ajuga Chamrpitys, Veronica precox, V. tiiphyllos, $V$. acinifolia, Pterolheca nemausensis; les bords des chemins : Iyosotis stricta, M. versicolor, Cynoglossum officinale, Saxifraga granulata, Cerastium brachypetalum et autres plantes triviales dont l'énumération n'offre aucun intérêt.

\section{b. Herborisation du Grand-Peupler a Margnolas \\ (GOMMUNE DE BEyNost)}

A partir de Miribel, le Rhòne s'écarte de plus en plus de la base de la côtière, en laissant une plaine alluviale qui va en s'élargissant jusqu'à la Valbonne; cette plaine est d'abord formée par les alluvions récentes du Rhône, puis par les terrasses alluviales dont nous avons parlé précédemment. (Voy. plus haut, p. 103).

Ces terrasses et les parties voisines de la plaine proprement dite sont constituées par des éléments semblables à ceux de la côtière, c'est-à-dire par des alluvions glaciaires, qui sont ou agglutinées en poudingues ou meubles, mais généralement mélangées au lehm ayant glissé des parties supérieures; aussi leur végétation estelle identique à celle du coteau.

En se dirigeant perpendiculairement sur la côtière, on trouve :

$1^{\circ}$ La plaine alluviale proprement dite, cultivee dans toute son ètendue. Les seules particularités botaniques qu'on puisse y relever sont : la fréquence du Berberis vulgaris et de l'Iris foetidissima dans les haies, du Veronica Buxbaumii, qui parait devenir de plus en plus commun dans nos environs, et les plantes méridionales qui envahissent les cultures, comme Plerotheca nemausensis, Barkhausia setosa devenues tout à fait spontanées, et Centaurea solstilialis, Helminthia echioicles, qui n'apparaissent que de temps à autre.

$2^{\circ}$ La terrasse alluviale sur laquelle sont situes les villages de Saint-Maurice et de Beynost. Elle est élevèe d'une sixaine de métres au-clessus de la plaine ; son flanc abrupt, formé d'alluvions gla- 
ciaires souvent consolidées en conglomérat, supporte une végétation intéressante analogue à celle des gravières de Neyron; on trouve en effet:

Echium vulgare.

Leucanthemum vulgatum.

Bupleurum falcatum.

Stachys recta.

Calamintha Nepeta.

Chondrilla juncea.

Lepidium graminifolium.

Amarantus retroflexus.

A. silvestris.

* Convolvulus cantabrica.

Setaria glauca.

Cerasus Mahaleb.

Dianthus prolifer.

Eryngium campestre.

Antirrhinum Orontium.
Reseda Phyteuma.

Eragrostis megastachya.

Teucrium Chamredrys.

Gypsophila Saxifraga.

* Barkhausia setosa.

Artemisia vulgaris.

Heliotropium europæum.

- Foniculum officinale.

Linum tenuifolium.

Asperula cynanchica.

* Lactuca saligna.

Caucalis daucoides.

* Lathyrus sphæricus (rare).

Scandix Pecten-Veneris.

Scilla autumnalis, etc.

Insistons surtout sur le Convolvulus cantabrica très abondant et qui reparaît plus loin sur une terrasse analogue s'ètendant de la Boisse à Balan et dans la Valbonne, sur le Fceniculum officinale qui se retrouve aussi tout le long de ces terrasses, à Miribel, Saint Maurice, Beynost, la Boisse, Balan, etc.

$3^{\circ}$ Le plateau de cette terrasse est occupé en cntier par des vil lages et des cultures; le sol, formé par un mélange de lehm et d'alluvions, est riche et de première qualitè.

$4^{\circ} \mathrm{La}$ base de la falaise est formée par les éboulis du conglomé rat; c'est une terre vegetale, renfermant plus ou moins de cailloux roulés, cultivè surtout en vignes et n'ayant comme Flure spontanée que les plantes triviales habituelles aux cultures, comme: Setaria viriclis, S. verticillata, Mercurialis anmua, Brassica nigra, Serratula arensis, Digitaria sanguinalis, Silene inflata Ranunculus repens, Chenopodium album, Ch. hybridum, Amarantus Blitum, A. retroflexus, Calendula arvensis, Potentilla reptans, Sonchus oleraceus, Caucalis segetum, Fumaria officinalis, Malva silvestris, Senerio vulgaris, etc. 
A u-dessus, la falaise est couronnée par des bois ou des broussailles reposant sur le conglomerat ou sur le lehm; leur Flore se compose de :

Quercus sessiliflora.

Cerasus Mahaleb.

Cornus sanguinea.

Prunus spinosa.

Ligustruin vulgare.

Coronilla Emerus.

Cratregus oxyacantha.

Acer campestre.

Evonymus europæus.

Ruscus aculeatus

Origanum vulgare.

Teucrium Chamedrys.

* Odontites lutea.

* Geranium sanguineum.

\author{
* Aster Amellus. \\ Galium corrudæfolium. \\ Veronica prostrata. \\ Picris hieracioides. \\ Coronilla varia. \\ Andropogon Ischæmum. \\ Caucalis segetum. \\ Brachypodium silvaticun. \\ Dianthus Carthusianorum. \\ Stachys recta. \\ Bupleurum falcatum. \\ Brunella vulgaris. \\ B. pinnatifida. \\ Helianthemum vulgare.
}

Quelques poudingues abrupts montrent: Cladonia divers, $B a r^{\text {- }}$ bula membranifolia, etc.

$5^{\circ}$ Enfin, au sommet, sur le lehm devenu siliceux, on voit apparaître: Cytisus capitatus, Sarothamnus vulgaris, Erica vulyaris, le Châtaignier, Andryala sinuata, Hieracium umbellatum et des Mousses nombreuses.

7. Si l'on poursuit sur le plateau, on arrive bientôt vers d'anciens étangs desséchés, vers le Ferrier, par exemple, où, sur la boue glaciaire, on trouve la Flore caractéristique de la Dombes :

Sagina patula.

Hypericum humifusum.

Gypsophila muralis,

Veronica arvensis.

Leoutodon autumualis.

Rumex Acetosella.

Gnaphalium luteo-album.

Gn. nliginosum.

Filago gallica.

F. germanica lutescens Jord.

F. canescens Jord.
Setaria glauca.

Anthoxauthum odoratun.

Jasione montana.

Trifolium arvense.

Matricaria inodora.

Viola segetalis.

Polygonum Persicaria.

P. lapathifolium.

P. Hydropiper.

Spergularia arrensis, etc. 


\section{E. Flore de la Valbonne 1}

" Toute la plaine de la Valbonne ne laisse voir que des cailloux provenant soit des alluvions anciennes ou glaciaires, soit des alluvions du Rhone postérieures au retrait des glaciers ${ }^{2}$. »

Mais, lor'squ'on se rapproche de Dagnieux et de Balan, le sol de cette partie de la Valbonne, de même que la terrasse de la Boisse dont elle est la continuation, est formé par le mélange des alluvions anciennes et récentes avec les terrains plus riches amenés par les cònes de déjection s'échappant des vallées de la Sereine et du Cotey. D'autre part, les collines de Saint-Maurice de Gourdan et de SaintJean de Niost, qui s'èlèvent dans le triangle formé par le confluent du Rhône et de l'Ain, sont constituées par les dépôts erratiques appartenant à ces « immenses moraines arquées autour du plateau oolithique de la Balme et de Crémieu ${ }^{3}$. »

Bien que les éléments siliceux dominent dans quelques parties, la végétation de la Valbonne, surtout sur ses bords, présente une grande ressemblance avec celle de la côtière méridionale de la Dombes.

Si l'on suit, en efiet, les balmes qui s'étendent de la Boisse au moulin Cassal, de ce moulin à Balan et de Balan à la ferme du Contant et à Pollet, on note :

Gypsophila saxifraga.

Linum tenuifolium.

Calamintha Nepeta.

Origanum vulgare.

Bupleurum falcatum.

Stachys recta.

Asperula cynanchica.

Thymus Serpyllum.

Echium vulgare.

Centaurea Calcitrapa.

Potentilla argentea.

Plantago Cynops.

Jasione montana.
Thesium divaricatum.

Tragus racemosus.

Torilis nodosa.

Silene Otites.

Coronilla minima.

Fœniculum officinale.

Convolvulus cantabrica.

Silene conica.

Carex montana.

Helianthemum pulverulentum.

H. salicifolium.

Ghlorocrepis staticifolia.

1 Ann. Soc. botan. de Lyon, t. 1, p. 122; - t. II, p. 87; - t. III, p. 73.

2 Falsan et Chantre. Catal. des blocs errat. dans Ann. Soc. Agric. de Lyon, l. $\mathrm{X}, 1877, \mathrm{p} .119$.

3 Falsan et Chantro Lonecit. 
Le sol rougeâtre de la plaine, généralement inculte, à cause de sa stérilité ${ }^{1}$, est caractérisé par une végétation mixte devenant en quelques endroits silicicole, par suite de la prédominance des éléments siliceux à la surface et de l'entrainement du carbonate de chaux dans la profondeur, comme l'indique l'abondance des cailloux de quartzites à la surface du sol; aussi celui-ci est-il couvert de : Andropogon Ischrmum, Trifolium arvense, Thrincia hirta, Jasione montana; mais on y rencontre aussi Ononis campes tris, Stachys recta, Plantago Cynops, Centurrea paniculata, etc., et dans quelques points Geranium sanguineum, Ajuga genevensis variété longistyla (Mathieu);

Plus près des collines qui s'étendent de Meximieux à SaintMaurice de Gourdans :
Helichrysum Stœchas.
Phleun arenarium.
Xeranthemum inapertum.
Alsine hybrida.
Kentrophyllum lanatum.
Orchis fragrans.
Galium corrudæfolium.
Cerastium arvense.
Veronica prostrata.
Carex Schreberi.
Ranunculus Chœrophyllos.
R. gramineus.

Ces collines renferment quelques plantes intéressantes, telles que l'Artemisia camphorata var. virgata Jord. et Four. (Sub Abrotano) et une nouvelle localité du rare Orchis papilionacea découverte par M. Fiard dans les pelouses et bois taillis de SaintMaurice de Gourdans, derrière le château de Marcelle.

Une autre plante remarquable, le Polygala exilis D C., espèce rare de la France méridionale, se rencontre dans plusieurs points de la Valbonne, surtout dans les endroits un peu humides, à Balan, aux Peupliers, etc.

Dans les terres cultivées:

Caucalis daucoides.

Gagea arvensis.

Pterotheca nemausensis.

Barkhausia setosa.
Crucianella angustifolia.

Valerianella coronata.

Linaria simplex.

1 La plaine de la Valbonne est occupee aujourd'hui en partie par le Camp de Balan. 
Et accidentellement : Senebiera Coronopus, Neslia paniculata, Vicia peregrina.

Au bord des chemins : Lepidium Draba, Stachys germanica, Myosotis stricta, Veronica Buxbaumii, etc.

Enfin, au delà de Meximieux, la station classique dite des Peupliers, explorée surtout par M. Chevrolat, renferme soit sur les coteaux secs, soit dans les prairies humides qui s'ètendent à leur pied, sur le bord de la rivière d'Ain : Pulsatilla rubra, Ononis Columnx, Cytisus argenteus, Trigonella monspeliaca, Bupleurum aristatum, Trinia vulgaris, Galiumitricorne, Artemisia virgata, Micropus erectus, Inula montana, Leontodon crispus, Neoltia autumnalis, Carex humilis., La présence de ces espèces, que nous avons vues pour la plupart en d'autres points de la còtière, montre bien que la végétation présente le même caractere depuis Lyon. On y trouve de plus: Polygala exilis, Spircea Filipendula, Scorzonera hirsuta, Chlora perfoliala, et autres espèces spéciales aux bords de la rivière d'Ain, ou que nous retrouverons sur les bords du Rhône, lorsque nous étudierons la Flore de ses alluvions récentes.

\section{1. - Énumération des Plantes d'après leurs \\ stations}

Si on réunit les indications disséminées dans les exemples d'herborisations qui précédent, en éliminant les espèces triviales, on peut établir ainsi qu'il suit la Flore des côtières occidentales et méridionales de la Dombes :

A. BoIs frais, lieuX ombragés

Espèces communes à toutes les èchancrures et dépressions du coteau :

Anemone nemorosa (purpurea).

Ranunculus nemorosus.

Aquilegia vulgaris.

Corydalis solida.
Viola Reichenbachiana.

V. Riviniana.

Mœhringia trinervia.

Oxalis Acetosella. 
Hypericum montanum.

Orobus tuberosus.

0 . niger.

Asperula odorata.

Melittis Melissophyllum.

Phyteuma spicatum

Primula grandiflora.

Galeopsis Tetrahit.

Pulmonaria tuherosa.

Euphorbia dulcis.

Paris quadrifolia,

Tamus communis.
Convallaria majalis

Scilla bifolia.

Luzula vernalis.

L. For'steri.

Scirpus silvaticus.

Carex pallescens .

C. muricata.

C, silvatica.

Melica nutans.

M. uniflora.

Aspidium aculeatum

Scolopendrium officinale.

Espèces spéciales:- vallon de Sathonay: Actra spicata, Sanicula europea, Mercurialis perennis, Maianthemum bifolium, Veronica montana (indiqué aussi à Meximieux, Cariot) : - Ca luire : Anemone ranunculoides; - l'Isopyrum thalictroides, à Meximieux et Reyrieux; - le Centaurea Ir.gdunensis, à la Pape; - le Dipsacus pilosus, à Trévoux, Reyrieux et dans le vallon de Sermenaz près Neyron; - l'Orchis papilionacea, à la Pape et Saint-Maurice de Gourdans.

\section{B. Pelouses sĖches, broussailles et talllis}

\section{Espèces communes :}

Ranunculus Chærophyllos.

Pulsatilla rubra.

Helleborus fœtidus.

Helianthemum salicifoliım.

H. guttatum.

Geranium sanguineum.

Linum gallicum.

Cytisus capitatus.

Ononis Columnæ.

Anthyllis Vulneraria.

Trifolium alpestre.

T. montanum.

Hippocrepis comosa.

Coronilla Emerus.

C. minima.

Cerasus Mahaleb.

Potentilla rupestris.

Peucedanum Cervaria.
Trinia vulgaris.

Galium corrudæfolium.

G. læve.

G. tricorne.

Rubia peregrina.

Globularia vulgaris.

Aster Amellus.

Inula montana.

Veronica prostrata.

V. præcox.

V. spicata.

Vincetoxicum officinale.

Campanula rapunculoides.

Lithospermum purpureo-cæruleun.

Teucrium montanum.

Euphrasia lutea.

Thesium divaricatum.

Ruscus aculeatus. 
Orchis Simia.

Ophrys anthropophora.

0. Morio.

0 . arachnites.

O. fusca.

Carex nitida.

O. ustulata.

C. Halleriaua.

0. pyramidalis.

Espèces spéciales: Scabiosa Gramuntia, Crupina vulgaris, Chrysocoma Linosy.jis, àla Pape;-Buxus sempervirens, Dianthus Scheuchzeri de la Pape à Miribel ; - Stipa pennata, Sathonay, la Pape; - Carex humilis, Hutchinsia petræa, Rhamnus saxatilis, Micropus erectus, Onosma arenarium, à Neyron et Meximieux (aux Peupliers) - Convolvulus cantabrica, Sathonay, la Pape, Neyron, et à partir de Miribel le long de la balme de la terrasse alluviale jusque dans la Valbonne; - Cornus mas. quelques rares pieds disséminés à Sathonay, rallon de la Cadette, etc.; - Limodorum aborlivum, à Fontaines, Neyrou; - Thalictrum aquilegifolium et Pulsatilla propera Jord., entre Montluel et Meximieux; - Aphyllanthes monspeliensis, entre la Pape et Montluel; - Glauwium luteum, au bourg Saint-Christophe; Cytisus biflorus et Cistus salvirefolius, seulement au-dessus de Neyron.

\section{C. Éroulis, gravières et SABLIÈres}

Erucastrum Pollichii.

E. obtusangulum.

Diplotaxis muralis.

D. tenuifolia.

Gypsophila saxifraga.

Dianthus prolifer.

Silene Otites.

S. conica.

Alyssum calycinum.

Liuum catharticum.

L. temuifolium.

Ononis Natrix.
Epilobium rosmarinifolium.

Scandix Pecten-Veneris.

Torilis nodosa.

Bupleurum aristatum.

Asperula cynanchica.

Centaurea paniculata.

Helichrysum Stœchas.

Chondrilla juxcea.

Anchusa italica.

Plantago Cynops.

Pl. arenaria.

Tragus racemosts.

Le Bromus madritensis, espèce méridionale qui ne s'éloigne pas des environs immédiats de Lyon; - Scabiosa sucueolens, 
à Meximieux; - Chlorocrepis staticifolia, plante dea éboulis du Jura et des Alpes, descendant par le Rhône sur les alluvions et les balmes de Balan, mais naturalisée sur les éboulis du conglomérat it Beynost.

\section{Cultures}

Fumaria parviflora. Caucalis daucoides: Pterotheca nemausensis. Barkhausia setosa. Centaurea solstitialis.
Helminthia echioides.

Neslia paniculata.

Iberis pinnata.

Odontites serotina.

Veronica Buxbaumii, etc.

Le Gladiolus segetum a été trouvé accidentellement dans les moissons a Saint-Maurice de Beynost.

Haies : en outre des espèces vulgaires, on y remarque fréquemment :

Berberis vulgaris.

Cerasus Mahaleb.

Cucubalus bacciferus.
Humulus Lupulus.

Iris fotidissima.

\section{8̧II. - Enumération systématique des espèces caractéristiques des coteaux du Rhone}

Ranunculus nemorosus. R. Chærophyllos, Pulsatilla rubra, P. propera, Anemone ranunculoides, A. nemorosa, Thalictrum aquilegifolium, Helleborus foetidus, Isopyrum thalictroides, Aquilegia vulgaris, Actrea spicata. - Berberis rulgaris.

Papaver Argenone, Glaucium luteum, Fumaria capreolata, F. parviflora, Corydalis solida.

Nasturtium silvestre, Erucastrum Pollichii, E. obtusangulum, Diplotaxis tenuifolia, D. muralis, Hutchinsia petræa, Iberis pinnata, Thlaspi perfoliatum, Neslia paniculata, Rapistrum rugosum.

Cistus salviæfolius, Helianthemum vulgare, H. obscurum, H. procumbens, H. pulverulentum, H. salicifolium, H. guttatum, H. canum.

Viola sp., Polygala vulgaris, Reseda Phyteuma.

Gypsoplhila saxifraga, Dianthus Armeria, D. Schcuchzeri, Silene Otites, S. italica, S. conica, Buffonia macrosperma, Alsine Jacquini, A. lyybrida, A. riscosa, Linum gallicum, L. tenuifolium, Hypericum montanum.

II alva moschata, Geranium sanguineum, Oxalis Acetosella, O. stricta. 
Rhamnus saxatilis, Rh. Frangula.

Sarothamuus scoparius, Genista anglica, G. germanica, G. pilosa, G. tincto1ia, Cytisus capitatus, C. biflorus, Ononis Natrix, O. Columnæ, Anthyllis Vulneraı ia, Trigonella monspeliaca, Melilotus arvensis, Trifolium medium, T. alpestre, T. rubens, T. ochroleucum, T. Molinerii, T. striatum, T. scabrum, T. subterraueum, T. glomeratum, Coronilla Emerus, C. minima, Hippocrepis comosa, Lathyrus hirsutus, L. sphreriens, L. latifolius, Orobus niger.

Cerasus Mahaleb, Fragaria collina, Potentilla r'upestris, Epilobium rosmarinifolium, Crassula r'ubens.

Caucalis dancoides, Torilis nodosa, Peucedanum Corvalia, Tordylium maxiv mum, Seseli coloratum, Trinia vulgaris, Foniculum officinale, Bupleurum aristatum, Bunium Bulbocastanum, Seandix Pecten, Sanicula europrea; - Cornus mas.

Sherardia arvensis, Asperula odorata, A. galioides, Rubia peregrina, Galium tricorne, G. corrulæfolium, G. Timeroyi, G. silvestre, Valerianella coronata, V. membranacea, V. microcarpa; Dipsacus pilosus, Scabiosa Gramuntia, S. suaveolens; Globularia vulgaris.

Cirsium acaule, Crupina vulgaris, Centaurea lugdunensis, C. paniculata, C. solstitialis, Kentrophyllum lanatum, Helichrysum Stoechas, Filago spathulata, F. montana, F. arreusis, F. gallica, Chrysocoma Linosyris, Artemisin virgata, Micropus erectus, Aster Amellus, Inula montana, I. hirta, I. salicina, Calenclula arvensis, Lactuca saligna, L. dubia, Chondrilla juncea, Pterotheca nenrausensis, Barlihausia setosa, Chlorocrepis staticifolia, Hieracium umbellatum, Audryala sinuatà, Leontodon hastilis, Leontodon crispus, Thrincia hirta. Helminthia echioides, Hypochreris maculata.

Jasione montana, Campanula Medium, C. persicifolia, C. rapunculoides.

Erica vulgaris, Primula grandiflora, P. variabilis, Lysimachia nemorum, Vincetoxicum officinale, Convolv:Ius cautabrica.

Physalis Alkekengi, Anchusa italica, Echinospermum Lappula, Myosotis stricta, Lithospermum purpureo-creruleum, Onosma arenarium;

Anarrhinum bellidifolium, Odontites serotina, O. lutea, Melampyrum arvense, M. pratense, Veronica montana, prostrata, spicata, Buxbaumii.

Thymus Chamædrys, Galeopsis Tetrahit, G. angustifolia, Stachys annua, Melittis Melissophyllum, Brunella grandiflora, Teucrium montanum.

Plantago Cynops, Daphne Laureola, Thesium divaricatum, Buxus scmper' virens, Euphorbia Gerardiana, Mercurialis perennis, Humulus Lupulus.

Paris quadrifolia, Ruscus aculeatus, Tamus communis, Maianthemum bifoliun, Arum italicum.

Lilium Martagon (au Veinay), Phalangium Liliago, Ph. ramosum, Scilla bifolia, Sc. autumnalis, Ornithogalum suliurenu, Allium sphærocephalum, A. vineale, $A$. intermediun, A. cariuatum, A. ursinum. 
Iris fotidissima, Gladiolus segetum?

Orehis hircina, $O$. bifolia, $O$. pyramidalis, 0 fusca, $O$. Simia, $O$. variegata ( $x$.), O. fragrans, O. Morio, O. rubra, Ophrys anthropophora, O. aranifera, 0 . fucifera, O. apifera, O. muscifera, Epipactis orata, E. lancifolia, Limodorum abortivum.

Aphyllanthes monspeliensis.

Scirpus silvaticus, Carex divulsa, C. Schreberi, C. remota, C. nitila, C. montana, G. Halleriana, C. humilis .

Stipa pennata, Kæhleria phlcoides, Melica ciliata, M. nutans, M. unilora, Poa megastachya, P. Eragrostis, Festnca rigida, F. tenuifolia, F. heterophylla, Bromus asper, B. gigantcus, B. squarıosus, B. maximus, B. madritensis, Nar' durus tenellus, N. Lachenalii.

Aspidium aculeatum, Scolopendrium officinale, Pteris arpuilina.

Barbula membranifolia, B. inclinata, B. squarrosa, Gylindrothecium concinum, Hypum rugosum, H. abietinum, Funaria calcarea, Psora decipiens, Psopoma fulgens, Thalloidima vesiculare, etc.

\section{\$IV. - Caracteres de la Flore des Falaises occidentale \\ et méridionale de la Dombes}

En résumé, voici quels sont les caractères qui distinguent la végétation de cette région de la Flore lyonnaise :

$1^{\circ}$ Absence des plantes aquatiques : Batrachium, Callitrichées, Haloragées, Potamogétonées, etc.

$2^{\circ}$ Rareté des plantes hygrophiles : Ce n'est que dans la profondeur des bois frais, autour des sources, le long des ruisseaux qui descendent par les échancrures du coteau et le long de la falaise, au niveau des marnes qui déterminent l'apparition des sources, qu'on observe quelques espèces hygrophiles, et encore sont-elles peu nombreuses :

Le long des ruisseaux, on ne rencontre que les especes triviales suivantes : Lupatorium cannabinum, Epilobium hirsulum, Galcopsis Tetrahit, etc.

Au nireau de; sources, sur les marnes des Boulées, prés de Miribel ; au même horizon au-dessus de Saint-Maurice, etc.: Phragmites communis, Eupatorium cannabinum, Equisetum, Sénecio Jacobcea, Solidago glabia, Imula dysenterica, Lathyrus latifolius. 
Dans les excavations des poudingues, qu'on observe en divers points du coteau, à Serin, par exemple, on trouve, inondées par des infiltrations d'eaux chargées de carbonate de chaux : Trichostomum tophaceum, Eucladium verticillatum, Gymnostomum curvirostrum, Hypnum commutatum, Vaucheria cæspitosa, etc. Nous verrons plus tard, dans d'autres points des coteaux du Rhône, aux Étroits, à Oullins, etc., de nouveaux exemples de cet habitat spécial concernant ces espèces éminemment calcicoles ${ }^{1}$.

3० Abondance des espèces psammiques : Les nombreux éboulis de sables, graviers et cailloux qui règnent tout le long du coteau, les pelouses qui les surmontent, renferment la plus grande partie des espèces qui caractérisent la Flore de cette région, ainsi qu'on peut le voir en se reportant aux énumérations données plus haut (Voy. pp. 118 et 120).

$4^{\circ}$ La présence d'un certain nombre de plantes méridionales : Grâce à l'exposition et peut-être aussi à la nature calcaire du sol, une colonie d'espèces dont l'habitat ordinaire est le Midi de la France, s'est établie sur plusieurs points de la côtière méridionale de la Dombes; voici les plus remarquables :

Cistus salviæfolius.

* Helianthemum salicifolium.

H. guttatum.

Cytisus biflorus.

C. argenteus.

Trigonella monspeliaca.

Crucianella angustifolia.

Valerianella coronata.

V. microcarpa.

Crupina vulgaris.
Xeranthemum inapertum.

Linosyris vulgaris.

* Convolvulus cantabrica.

Kentrophyllum lanatum.

Onosma arenarium.

Aphyllanthes monspeliensis.

Orchis fragrans.

0 . papilionacea.

* Barbula membranifolia.

Plusieurs de ces espèces (celles marquées d’un *) se retrouven sur les versants exposés au Midi, de la còtière occidentale.

1 Ces mêmes espèces se retrouvent aussi sur les tufs calcaires du Bugey et du Jura; à propos de ces derniers, M. Contejean (Ann.sc. natur., 1875, t. II, p. 12i), donne une énumération qui offre beaucoup d'analogie avec la nôtre; nons faisons cependant des réserves pour le Systegium crispum, qui, dans nos environs du moins, non sculement ne croît jas en compagnie des Mousses précédemment citées sur les ircrustations tufeuses des poudingues. mais paraît au contraire prefẻrer les sols granitiques. 
Nous verrons plus tard, dans le chapitre consacré plus spécialement à cette étude, que cette Flore méridionale existe pareillement au Mont-d'Or, sur plusieurs points des Balmes-Viennoises, et dans les parties bien exposées des vallées du Bugey et de la Savoie, c'est-à-dire dans des régions calcaires, d'où l'on peut déjà conclure qu'à l'influence de l'exposition s'ajoute celle de la nature du sol ${ }^{1}$.

$5^{\circ}$ La prédominance marquée des aspèces calcicoles : Du dẻpouillement de nos énumérations il ressort que, si l'on met de côté les espèces indifférentes, il reste un nombre bien plus grand d'espèces calcicoles que de silicicoles.

On trouve, en effet, comme espèces plus ou moins adhérentes :

* Helianthemum procumbens.

H. Fumana.

* H. pulverulentum.

H. salicifolium.

Dianthus Scheuchzeri.

Rhamnus saxatilis.

* Ononis Columnæ.

* 0 . Natrix.

Trifolium alpestre.

T. rubens.

* Coronilla Emerus.

C. minima.

* Hippocrepis comosa.

Lathyrus latifolius.

* Trinia vulgaris.

Cornus mas.

Asperula galioides.

Rubia peregrina.
Galium corrudæfoliun.

* Aster Amellus.

* Inula montana.

* Vincetoxicum officinale.

* Lithospermun purpureo-cæruleum.

* Brunella grandiflora.

* Telcrium montanum.

* Daphne Laureola.

* Buxus sempervirens.

* Mercurialis perennis.

* Orchis hircina.

* 0 . pyramidalis.

* Ophrys muscifera.

* 0 . anthropophora.

* Carex gynobasis (Halleriana).

* C. humilis.

* Stipa pennata.

* Melica uniflora.

1 Nous ne disons pas que toutes ces espéces méridionales sont des plantes calcicoles ; quelques-unes croissent manifestement, dans d'autres localitès, sur les gneiss: mais nous constatons de nouveau que les plantes du Midi remontent plus haut (en latitude) dans les régions calcaires que dans les régions siliceuses, ce qui tient évidemment à ce que les sols calcaires ont une température moyenne annuelle plus èlevée que les silicenx, comme le prouvent les températures des sources, l'époque des moissons et d'autres données agricoles (Voy. plus haut, p. 35).

On peut rapprocher de ce fait la clécouverte d'une Fanuule m lacologique de trois espèces méridionales (Ielix trochoides, H. acuta, Pupa quinquedentata) trou. vées précisement sous la Pape, et que vient de décrire M. Locard (Ann. Soc. ('agric. Lyon, t. X, 187i. p. 96). 
Plantes adhérentes, mais rares:

* Thalictrum aquilegifoliım. Hutchinsia petræa.

Helianthenum canum.
Buffonia macrosperma

Alsine Jacquini.

* Chrysocoma Linosyris.

Plantes préférentes, peu adhérentes:

Berberis vulgaris.

Diplotaxis tenuifolia.

Iberis pinnata.

Reseda Phyteuma.

Neslia paniculata.

Rapistrum rugosum.

Silene italica.

Linum tenuifolium.

* Antlyyllis Vulneraria.

Otrobus niger.

* Cerasus Mahaleb.

Lathyrus sphæricus.

Fragaria collina.

Caucalis daucoides.

Peucedanum Cervaria.

Seseli coloratum.

Bupleırum aristatum.
Scandlix Pecten.

* Globularia vulgaris.

Kentrophyllum lanatum.

Micropus erectus.

Lactuca saligna.

Galeopsis Tetrahit.

* Stachys recta.

Plantago Crnops.

Iris foetidissima.

Ophrys sp.

Epipactis sp.

Limodorum abortivum.

* Phalangium ramosum.

* Ornithogalum sulfureum.

Carex nitida.

Aphyllanthes monspeliensis.

Soit 41 adhérentes et 35 préférentes.

Toutes ces espèces se retrouvent dans le Mont-d'Or et dans le Bugey.

Sur ces 71 espèces, 33 sont données comme caractéristiques des sols calcaires par M. Contejean; encore n'avons-nous pas compté les Helleborus futidus, Bupleurum falcatum, Teucrium Chamædrys, Melica ciliata, considérées comme adhérentes par M. Contejean, mais que leur dispersion dans le domaine de la Flore lyonnaise nous fait regarder comme peu caractéristiques.

Nous pourrions ajouter aussi les Barbula membranifolia, Hypnum rugosum, les Gymnostomum curvirostrum, Eucladium verlicillatum, Trichostomum tophaceum, Hypnum commuta${ }^{t} u m$, tout a fait adhėrents, mais qui croissent sur un substratum spécial, manifestement calcaire, le ciment ou les dépôts tufeux des poudingues du conglomérat. 
Or, en regard de ce chiffre de plus de 70 espèces calcicoles, nous ne trouvons qu'une dizaine de plantes préférant les sols siliceux, et encore croissent-elles certainement dans des terrains dépourvus de carbonate de chaux, ce sont :

Malra moschata.

Sarothamnus seoparius.

Filago sp.

Hieracium umbellatum.

Andryala sinuata.

Thrincia hirta.
Jasione montana

Erica vulgaris.

Anarrhinum bellidifolium.

Festuca heterophylla.

Nardurus Lachenalii.

Pteris aquilina.

Et, par contre, il convient d'insister sur l'absence complète des plantes silicicoles caractéristiques suivantes, si communes cependant à peu de distance du bord de la côtière, dans la Dombes sili ceuse : Gypsophila muralis, les Sagines, les Spergules, Stellaria uliginosa, Radiola linoides, Hypericum humifusum, H. pulchrum, Ulex europaeus, Corrigiola littoralis, Illecebrum verticillatum, Arnoseris minima, Galeopsis ochroleuca, etc. 



\section{APPENDIGE}

Ainsi que le montre le tableau des différentes parties dont ces Recherches doivent se composer (voyez précédemment page 23), il entrait dans mon plan primitif de publier, en même temps et avec les mêmes développements que je viens d'apporter à l'étude des bas-plateaux lyonnais et de la côtière méridionale de la Dombes, les autres coteaux du Rhône, le Mont-d'Or et le plateau bressan. Mais l'ètendue considérable donnée à cette première partie de mon travail et la nécessité où je suis de la publier de suite, me forcent à ajouter dès maintenant, en appendice, les résultats principaux de mes observations phytostatiques sur la région lyonnaise, en les faisant précéder d'une revue historique des travaux les plus importants publiés sur les rapports de la végétation avec la nature du sol.

\section{GHAPITRE PREMIER}

DE L'INFLUENCE GHIMIQUE DU SOL SUR LA VÉGETATION.

COUP-D'AEIL HISTORIQUE

Il serait trop ambitieux, à propos d'une étude sur la géographie botanique du Lyonnais, de faire l'histoire complète des travaux relatifs à la question des rapports qui existent entre la végétation spontanée et la nature minéralogique du sol. Je me bornerai donc à rappeler brièvement les plus importants de ces travaux.

1 Cette partie de notre travail a été rédigée sur les notes et les nombreux renseignements commaniqués par notre obligeant ami, M. le $\mathrm{D}^{r}$ Saint-Lager. 
Déjà les anciens botanistes avaient remarqué l'influence que le sol exerce sur la distribution des plantes, comme le prouve l'aphorisme bien connu de Linné : dignoscitur ex sola inspectione plantarum subjecta terra et solum ${ }^{1}$.

A la fin du siècle dernier, Link fit une étude particuliere de la Flore des environs de Göttingen, sous le rapport géognostique, et signala les espéces propres aux sols calcaires, lesquelles sont complètement diffèrentes de celles qu'on observe sur les terrains sablonneux et argileux ${ }^{2}$.

Wahlenberg avait remarqué que, dans la chaine des Carpathes, il est des espèces qui ne croissent que sur le granite, d'autres, au contraire, qui ne viennent que sur les roches calcaires ${ }^{3}$.

En 1825, de Caumont signalait le contraste très prononcé qui existe entre la Flore des régions calcaires de la Normandie et celle des territoires non calcaires du même pays ${ }^{4}$.

Alph. de Brébisson ${ }^{5}$ et Dubourg d'Isigny ${ }^{6}$ développèrent la donnée que de Caumont n'avait fait qu'esquisser.

Dans l'ètude qu'il avait faite de la Flore de Brunswick, Lachmann était arrivé aux mêmes conclusions et avait constaté que la végétation des calcaires du Muschellialk est toute différente de celle des argiles du Keuper et des sables produits par la désagrégation des grès bigarrés ${ }^{7}$.

Dans un ouvrage concernant l'histoire de l'Autriche (unter der Enns)" publié à Vienne en 1832, on trouve un remarquable article intitulé : Daistellung der Pflanzen-geographischen Ver"hältnisse (Exposé de la distribution géographique des plantes), par Johann Zahlbruckner.

1 Philos. botan., § 334.

2 Flore Gœttingensis specimen, sistens vegetabilia saxo calcareo propria. Gœettingen, 1789.

3 Flora Carpatorum principalium. Gottingen, 1814.

4 Essai sur la topographie géognostique du Calvados; dans Mém. Soc. linn. du Calvados, t. IV.

5 Coup d'orl sur la végétation de la Basse-Normandie considérée dans ses rap. ports avec le sol et les terrains, $i b i d$.

- Aperçus sur la géologie et la Flore de l'arrondissement de Vire, 1836.

7 Flora Brunsvicensis. Braunschweig, 1828.

\& Beitrege zu' Landeskunde (Esterreichs, unter der Euns. Wien, 1832. 
L'auteur énumère les principales espèces qni habitent exclusirement, soit les montagnes calcaires, soit les montagnes primitives.

En 1836, parut à Vienne un ouvrage qui marque une étape importante dans l'histoire de la Phytostatique ${ }^{1}$. Unger, professeur de botanique à Grätz, publia le résultat de ses observations sur la distribution des plantes dans la partie N.-E. du Tyrol, et spécialement dans le district de Kitzbühel.

Sous le rapport géognostique, il divisait les plantes en :

$1^{\circ}$ Kalkstete (spéciales à la chaux);

$2^{\circ}$ Kalkholde (amies de la chaux);

$3^{\circ}$ Schieferstete (spéciales aux schistes);

$4^{\circ}$ Schieferholde (amies des schistes);

$5^{\circ}$ Bodenwage (indifférentes au sol).

Les expressions de Schieferstete et de Schieferholde ne tarderent pas à être remplacées par celles de Kieselstete et de Kieselholde, attendu que la végétation des schistes micacés et argileux offre une grande ressemblance avec celle des gneiss, granites et grès, et que dans ces divers terrains il y a un élément commun, la silice (Kiesel) combinèe avec l'alumine et les alcalis.

Ainsi modifiée, la classification d' Unger fut généralement adoptée et n'avait plus besoin que d'un complément, c'est-à-dire l'adjonction d'une sixième catégorie, celles des halophiles (amies du sel, Salzholde).

Les recherches d'Unger ne portaient que sur une partie des Alpcs tyroliennes; Hugo de Mohl les continua, en les étendant à toute la chaine des Alpes autrichiennes et helvétiques. Dans son mémoire? H. de Mohl énumère 752 espèces alpines, qu'il répartit en cinq catégories correspondant à celles déjà établies par Unger.

En 1843, Grabowski fit le même travail pour la Silésie et les montagnes voisines ${ }^{3}$.

1 Ueber den Einfluss des Bodens auf die Vertheilung der Gewächse (Influence du sol sur la distribution des plantes). Wien, 1836.

2 Ueber den Ëinfluss des Bodens auf die Vertheilung der Alpenpflanzen. Disser. tation de 1838, rẻimprimée dans les Vermischte Schriften. Tübingen, 1846.

3 Flora der Olerschlesien u. d. Gesenke. Breslau, $18: 3$. 
A l'occasion des opinions contradictoires qui avaient été émises à la réunion extraordinaire de la Société géoløgique de France à Chambéry, Ch. Desmoulins fit paraître, en 1846, 1847 et 1848, trois mémoires dans lesquels il discuta la question de l'influence minéralogique et chimique du sol sur la végétation ${ }^{1}$.

A la même époque, Mougeot, qui avait fait une ètude attentive de la végétation de la chaîne vosgienne, publiait le résultat de $\mathrm{se}_{\mathrm{S}}$ observations; en regard du nom de chaque espèce, il indiquait l'habitat géologique ${ }^{2}$.

Les travaux de Mougeot complétaient ceux qui avaient étẻ déjà si heureusement entrepris par M. Godron ${ }^{3}$.

En 1848, Schnizlein et Frickinger mettaient en lumière le con-traste frappant qui existe entre la végetation des calcaires jurassiques et celle des grès bigarrés et des argiles keupériennes de la Franconie ${ }^{4}$.

En 1849, Thurmann, déjà connu par d’importants travaux géologiques, publia un ouvrage dans lequel il exposait et groupait, avec un remarquable talent, toutes les observations phytostatiques faites, d'une part; dans la chaîne des Vosges et le Schwarzwald, d'autre part, dans les territoires calcaires de l'Alb Wurtembergeois, de la Lorraine, de la Côte-d'Or, de la chaîne jurassique et des montagnes calcaires de la Savoie et du Dauphiné ${ }^{5}$.

Thurmann démontra avec la plus complète évidence que, dans la vaste étendue de pays qu'il considerait et, abstraction faite des conditions climatériques, la végétation est en relation directe avec

1 Examen des causes qui paraissent influer particulièrement sur la croissance de certains végétaux; dans t. I des Mém. de l'Institut des prov., 1847; - $2^{\circ}$ mémoire dans t. XV, 1848, Actes Soc. linn. de Bordeaux; $-3^{\circ}$ mem., ibid.

2 Consilérations générales sur la végétation des Vosges. Épinal, $18 i ́$.

3 Flore de Lorraine. Nancy, 1842. Cet ouvrage a étè réedite en 1857 et contient de prècieux renseignements sur les stations géologiques des plantes. On trouve aussi d'utiles indications, relativement au sujet qui nous occupe, dans la Flore d'Alsace par Kirschleger, Strasbourg, 1852 et surtout dans l'Essai sur la Géographie bota. nique de la Lorraine, par M. Godron, Nancy, 1862.

+ Die Vegetations-Verhältnisse der Jura und Keuperformation in den Flussgebieten der Wornits und Altmühl. Nordlingen, 1848.

5 Essai de Phytostatique applique à la chaîne dn Jura et aux contrées voisines. 2 vol. Berne, $184 \%$. 
la nature minéralogique des terrains. Il réduisait donc à néant cette singulière assertion de l'illustre de Candolle qui avait dit que " la végétation des montagnes de granite et de grès des Vosges ne diffère pas sensiblement de celle des montagnes calcaires du Jura. On ne pourrait pas citer une seule plante qu'on n'ait trouvée exclusivement que sur les sols calcaires ou sur 'les sols granitiques ${ }^{1}$. $)$

Thurmann, si habile dans l'exposition des faits, fut moins heureux dans leur interprétation. Il nia presque complètement l'action chimique des roches, ou du moins ne l'admit que lorsque celles-ci contenaient des sels très solubles comme le sel marin ou les sels ammoniacaux. Pour lui, l'influence du sol sur la végétation était, dans la plupart des cas, sous la dépendance du mode de désagrégation des roches et de leurs propriétés physiques.

La doctrine de Thurmann fut accueillie avec grande faveur; elle fut acceptée et défendue par plusieurs naturalistes parmi lesquels il suffira de citer, pour le moment, MM, Contejean ${ }^{2}$, Delbos ${ }^{3}$, Alph. le Candolle ${ }^{4}$, Wirtgen ${ }^{5}$, Hoffmann ${ }^{6}$. .

Cependant, de toutes parts des protestations ne tardèrent pas a s'élever contre une doctrine qui ne laissait aucune part d'influence, sur la distribution des plantes, aux qualités chimiques des roches sous-jacentes. Beaucoup de bons observateurs se refusèrent a ne voir dans le sol qu'un simple support mécanique. Parmi eux, citons en particulier Boreau ${ }^{7}$, Bogenhard ${ }^{8}$, Lècoq ${ }^{9}$, M. Em.

1 Dict. des sc. natur., art. Géogr. bot., t. XXIII. Physiologie végétale, t. III.

2 Enumérat. des pl. vasc. de Montbéliard. Besançon, 1854, dans Mém. Sc. d'Emul. du Doubs.

${ }^{3}$ Recherches sur la répartition des végét. dans le département de la Gironde dans t. I, Mém. Soc. sc. phys. nat. Bordeaux. 1855.

4 Gèographie botanique, t. I, p. 422. Genève, 1855.

5 Flora d. Rheinprovinzen. Bonn, dans Verhandl. d. naturh. Ver. d. Preuss. Rheinlande, 1853-5\%.

- Ueber die Vegetation d. hohen u. d. vulcanischen Eifel. ibid, t. XXII, 1865.

${ }^{6}$ Vergleich. Studien zur Lehre v. d. Bodenstetigkeit d. Pflanzen, dans $S^{\circ}$ Bericht d. Oberhess. Gesellsch., Giessen, 1860. - Untersuchungen zur Lïlima und Boden kunde mit Rücksicht auf die Vegetation, dans Botan. Zeitung, 1865.

7 Flore du Centre de la France. Paris, 1819.

8 Taschenbuch d. Flora v, Jena. Leipzig. 1850.

${ }^{3}$ Études sur la Géographie Jotanique de l'Europe, t. II, p. 45. Paris, 1854 
Planchon ${ }^{1}$, Sendtner ${ }^{2}$, Stur ${ }^{3}$, Parisot ${ }^{4}$, Lejolis ${ }^{5}$, Godron ${ }^{6}$, Boulay ${ }^{7}$.

Par un revirement d'opinion qui fait le plus grand honneur à sa bonne foi, M. Contejean, qui avait été un des plus chauds partisans de la doctrine de son maître Thurmann, s'est rangé, depuis quelques années, parmi ses adversaires les plus convaincus. Dans une série de Mémoires, il a combattu victorieusement les arguments de Thurmann et a apporté des arguments en faveur de la doctrine qui attrribue la dispersion des espèces végétales principalement à l'influence chimique des roches sous-jacentes ${ }^{8}$.

La science forestière est venue aussi apporter son contingent à la question de l'action chimique du sol sur les plantes.

Déjà Giraud--Soulavie, dans un ouvrage peu connu et généra!ement nassé sous silence, bien qu'il renferme sur les régétations différentes desterrains calcaires et siliceux, des observations remarquables pour l'époque, avait, dès 1780, constaté que les Châtaigniers s'arrêtaient où le sol sablonneux devient calcaire $\left(2^{\mathrm{e}} \mathrm{par}-\right.$ tie, chap. viII, p. 149) ${ }^{9}$.

Dunal, en s'aidant des indications géologiques qui lui avaient été données par M. Paul de Rouville et par E. Dumas, réfuta certaines

1 Sur la végétation spéciale des dolomies du Gard et de l'Hérault. Bull. Soc. bot. Fr., t. I, 1854. - Sur la végétation des terr. siliceux du Gard et de l'Herault, ibid. Compte rendu de la quinzième session de la Soc. helv. de sc. nat. Lausanne, $18 j 1$.

2 Die Vegetations-Verhältnisse Sudbayerns. München, 1854.

3 Ueber d. Einfluss d. Bodens auf d. Vertheilung d. Pflanzen. t. XX et XXV Sitzber. d. k. Akad. d. Wiss. Wien, 185̄6-57.

4 Note sur la Flore des envir. de Belfort. Mém. Soc. émul. Doubs, t. III de $3^{\circ}$ sér. Besançon, 1858.- Rapport sur une herborisat. au Kaiserstuhl. Bull. Soc. bot. Fr., t. Y, 1858.

5 De l'influence chimique des terrains sur la dispersion des plantes, t. VIII, 1861. Mém. Soc. sc. nat. de Cherbourg. - La soude dans le sol et les régétaux. Comptes rendus Acad.sc., t. LXXXVI, 1878.

6 De la végétation du Kaiserstuhl. Nancy, 1864. - De l'Espèce et des Races, t. I, p. 91. Paris, 1859.

7 Notice sur la Géographie botau. des envir. de Saint-Dié. Besançon, 1866.

8 De linfluence du terrain sur la végétation. Ann. d. sc. nat. $5^{\circ}$ sèr. t. XV p. $266^{2}$. Ibid., $6 \bullet$ série, t. II.

${ }^{\circ}$ Histoire naturelle de la France méridionale. Paris, MDCCCLXXX, 8 vol. 2' partıe, chap. vill; 3' partie, Ch. I, 11 et 111 surtout. 
erreurs relatives à la dispersion de cet arbre. Il montrait que, dans la vallée de l'Hérault, autour de Saint-Guilhem le Désert, le Châtaignier croit sur des couches de calcaire oolithique à entroques recouvertes de nodules siliceux qui fournissent à cet arbre l'élément chimique dont il a besoin ${ }^{2}$.

M.M. Fliche et Grandeau ont aussi fait une étude approfondie de l'influence chimique du sol sur la croissance du Châtaignier et du Pin maritime ${ }^{2}$.

Enfin, s'appuyant sur des considérations tirées de la chimie physiologique et de l'expérience agricole, mon ami, M. le docteur Saint-Lager ${ }^{3}$ est venu apporter des arguments décisifs en faveur de la doctrine de l'influence chimique du sol. En effet, dit-il, puisque certains composés minèraux sont indispensables à la vie des plantes au même titre que le phosphate de chaux, le sel marin et le fer à la vie d'un grand nombre d'animaux, puisque les quantités de ces composés minéraux sont fort différentes dans les divers terrains, puisque enfin l'appétit des plantes pour chaque substance minérale est lui-même variable suivant les espèces, ainsi que le démontrent les analyses de cendres et les faits agricoles, on est nécessairement conduit à adınettre que la distribution géographique des végétaux est en rapport direct avec la facilité que ceux-ci trouvent sur tel ou tel sol à satisfaire leurs besoins.

J'aurais encore à citer les ourrages lichénographiques, bryolo giques et algologiques concernant les relations qui existent entre la nature chimique du support et la distribution géographique des Lichens, des Mousses et des Algues.

C'est un dogme accepté par tous les lichénographes, depuis Acharius et Fries jusqu’à Kiærber et Nylander que les Lichens saxicoles sont presque tous fixés invariablement, les uns sur les roches siliceuses, les autres sur les pierres calcaires ${ }^{4}$.

1 De l'influence minéralogique du sol sur la végétation. Mém. Acad. de Montpellier, sect. sc. t. I, p. 174, 1848.

2 De linfluence de la compos. chim. du sol sur la végèt. du Pin marit. et du Chàtaignier. Ann. phys. chimie, t. XXIX de $4^{\circ}$ série, 1873 ; t. II de $5^{\circ}$ série, 1874.

3 De l'intluence chimique du sol sur les plantes. Ann. Soc. bot. Lyon, t. T, 1876-iт. Note sur la Géogr. bot. de la Bresse et de la Limagne d'Aurergne, ibid. t. VI, 18it-78.

1 Cf. Weddel, 
Il me faudrait citer aussi les nombreux ouvrages des bryologues qui se sont appliqués à signaler la diversitẻ des Mousses vivant sur les rochers de granites, de gneiss, de grès, arrosés par des eaux pures, d'avec celles qui s'attachent aux pierres mouillees par des eaux calcaires ${ }^{1}$.

Enfin, il me resterait encore à mentionner les remarquables études faites sur les Diatomées silicivores, ainsi que sur les Desmidiées calcivores.

Mais une pareille énumération m'entraînerait au delà des bornes assignées à mon modeste travail. Aussi, je termine là l'historique déjà trop long des ouvrages relatifs à la question de l'influence physico-chimique du sol sur les plantes.

\section{CHAPITRE II}

ANALOGIES ET CONTRASTES PRÉSENTÉS PAR LES DIVERSES PARTIES DE LA REGION LYONNAISE

COMPAREES ENTRE ELLES OU AVEC LES REGIONS VOISINES

\$I. Comparaison de la végétation du Mont-dor lyonnais avec celle des montagnes du Jura, du Bugey et de Crémieu

L'influence que la nature minéralogique et chimique des roches exerce sur la régétation est démontrée avec la plus complète évidence par la ressemblance qui existe, à altitude égale, entre la Flore du Mont-d'Or lyonnais et celle des chaines calcaires du Jura, du Bugey, et des pays de Crémieu et de Morestel².

Il serait trop long de donner ici l'énumèration des plantes com-

1 Cf. Boulay.

2 On sait que les montagues des environs de Grémieu et de Morestel, situées dans le nord du departement de l'Isère, ne sont séparées de celles du Bugey que par la cassure à travers laquelle coule le Rhòne et, comme ces dernières, appartiennent a la formation jurassique. 
munes aux deux régions jurassiques; je me bornerai à présenter la liste des espèces les plus caractéristiques .

Thlaspi perfoliatum.

Polygala comosa.

Dianthus silvestris.

Acer monspessulanun.

A. opulifolium.

Cytisus Laburnum.

Ononis Columnæ.

Trifolium rubens.

T. alpestre.

Vicia tenuifolia.

Coronilla Emerus.

C. minima.

Hippocrepis comosa.

Cerasus Mahaleb.

Trinia vulgaris.

Cornus mas.

Bunium Carvi.

Lonicera etrusca.

Galium Timeroyi.

Rubia peregrina.

Centaurea montana (v. lugdunensis).

Pyrethrum corymbosum.

Inula montana.

Campanula glomerata.

Gentiana Cruciata.

G. ciliata.

Anchusa italica.

Lithosperm. purpureo-cæruleum.

Veronica spicata.

Digitalis parviflora.
Galeopsis angustifolia.

Stachys annua.

Brunella grandiffora.

Rumex scutatus.

Daphne Laureola.

Aristolochia Clematitis.

Buxus sempervirens.

Mercurialis perennis.

Ornithogalum sulfureum.

Orchis pyramidalis.

0 . fusca.

O. Simia.

Aceras hircina.

Ophrys fucifera.

O. muscifera.

O. anthropophora.

Epipactis lancifolia.

E. rubra.

Limodorum abortivum.

Alopecur'us agrestis.

Polypodium calcareum.

Melica uniflora.

Carex alba.

C. montana.

C. gynobasis.

C. ornithopoda.

Seligeria pusilla.

Grimmia crinita.

Placodium callopismum.

Verrucaria calciseda, etc.

La plupart de ces espèces ont été données comme caractéristiques par les auteursqui se sont occupés de la question des relations du sol arec la végétation. En effet, si nous consultons les Etudes de Gégraphie botanique de Lecoq, nous voyons que les Thlaspi perfoliatum, Polygala comosa, Althaca hirsuta, Acer opulifolium, Ononis Columnæ, Trifolium rubens, Vicia tenuifolia, Coronilla Emerus, C. minima, Hippocrepis comosa, Trinia vulgaris, Cornus mas, Lonicera etrusca, Rubia peregrina, Pyrethrum 
corymbosum, Campanula glomerata, Gentiana ciliata, Ancliusa italica, Stachys annua, Rumex scutatus, Aristolochia Clematitis, Omithogalum sulfureum, Orchis fusca, O. hircina, Ophrys muscifera, anthropophora, Carex gynobasis, Polypodium calcareum, appartenant à notre liste, sont données comme calcicoles par cet auteur.

Et M. Contejean range parmi les espèces préférant les sols calcaires : Acer opulifolium, Ononis Columnæ, Coronilla Emems, C. minima, Hippocrepis comosa, Cerasus Mahaleb, Trinia vulgaris, Inula montana, Gentiana Cmuciata, Lithospermum purpureo-crmuleum, Digitalis parviflora, Brunella grandiflora, Rumex scutatus, Daphne Laureola, Buxus sempervirens, Mercurialis perennis, Orchis pyramidalis, O. hircina, Ophrys muscifera, O. anthropophora, Epipactis mbra, Carex alba, C. gymobasis, C. omithopoda, Melica uniflora, Polypodium calcareum.

Les autres espèces sont des calcicoles moin exclusives comme Acei monspessulanum, Veronica spicata, etc., ou des espèces qui sont pour nous manifestement calcicoles, du moins dans notre région, telles que Dianthus silvestris, Comis mas, Rubia peregrina, Inula montana, etc.; nous les avons toujours vues, en effet, sur les calcaires ou les terrains riches en carbonate de chaux, comme les alluvions ou les mollasses calcaires des coteaux du Rhône.

Cependant parmi les espèces qui sont reconnues comme préfé-. rentes ou exclusives calcicoles par Lecoq, Contejean, etc. et par nous, quelques-unes peuvent se trouver accidentellement dans les sols siliceux; ce sont par exemple l'Helleborus foeticus et le Dentaria pinnata, que nous n'avons pas cités dans notre liste, bien qu'ils se trouvent abondamment dans les régions que nous étudions dans ce paragraphe; ces espèces sont données comme calcicoles par beaucoup d'auteurs et notamment par M. Contejean ; or, nous avons vu l'Hellébore fétide très abondant sur les gneiss du Lyonnais (Voy. précédemment p. 80) et nous-mème avons trouvé le Dentaria pinnata dans les montagnes du Lyonnais, en plein terrain siliceux, mais, il est vrai, sur as porphyres, qui renferment souvent une 
proportion variable d'élément calcaire ${ }^{1}$. Il en est de même du Mercurialis perennis, que j'ai observé, sur les gneiss, dans les bois du Lyonnais, et du Rumex sculatus, qui paraît de prime abord si caractéristique des régions calcaires, qui est reconnu comme tel par les botanistes statisticiens et qu'on trouve cependant sur les micaschistes du Pilat.

L'espace nous manque pour développer ces considérations; nous les reprendrons avec plus de détails dans la partie que nous comptons consacrer prochainement à l'étude complète des végétations calcicoles et silicicoles.

Après avoir indiqué les similitudes, il ne sera pas inutile de faire ressortir les différences qu'on observe entre la Flore du Mont-d'Or lyonnais et celle de la chaîne jurassique.

L'altitude maximum de notre Mont-d'Or étant de 612 mètres au mont Toux et de 625 mètres au mont Verdun, il est bien évident que les espèces végétales propres à la région alpestre du Jura manquent aux sommités du Mont-d'Or lyonnais.

On ne sera pas surpris non plus de l'absence des nombreuses plantes vivant en société dans les forêts de Sapins qui couvrent les pentes de la plaine jurassique de 650 à 1,000 mètres.

Le défaut d'altitude n'est d'ailleurs pas la seule cause qui s'oppose à l'établissement des forêts de Sapins dans notre Mont-d'Or : celles-ci ont besoin, pour leur bonne venue, d'un sous-sol frais et humide. Or les sources du Mont-d'Or lyonnais se trouvent toutes à un niveau relativement bas, qui est celui des marnes supérieures du lias, comme il est facile de le voir à Collonges, à Saint-Romain, à Couzon, à Curis, à Poleymieux et à Saint-Germain. Toute la masse des calcaires situés au-dessus de ce niveau est très fendillée et laisse rapidement écouler les eaux. Il n'existe au Mont-d'Or que quelques buis de peu d'importance. Au surplus, on sait que dans le voisi-

1 Plusieurs auteurs ont signalé la présence d'espéces calcicoles sur les porphyres: en attendant que nous développions ce point particulièrement intẻressant des exceptions apparentes à la théorie de l'influence chimique du sol, uous renvoyous an travail de M. Legrand (Stat. botan. du Forez, p. 44) qui indique aussi le Dentaria pinnata en socièté d'autres préfërentes calcicoles (Ophrys apifera, Helianthemum Fumana etc.) sur les porphỵres de la Loire. 
nage des grandes villes, la terre, ayant une valeur relativement élevée, estemployée de préférence à la culture productive de la vigne et des plantes fourragères.

Toutefois on est surpris, au premier abord, de ne pas rencontrer dans notre Mont-d'Or plusieurs espèces calcicoles qui, dans la chaîne jurassique, descendent au-dessous de la région des Sapins et qu'on trouve même à partir de 350 mètres. Parmi ces espèces, nous citerons en particulier :

Kernera saxatilis, Ethionema saxatile, Sisymbrium austriacum, Clypeola Jonthlaspi, Arabis alpina, A. auriculata, Lunaria rediviva, Anthyllis montana, Potentilla caulescens. Cotoneaster vulgaris et tomentosa, Amelanchier vulgaris, Rhamnus alpina, Laserpitium Siler, Valeriana montana, Carduus defloratus, Lactuca perennis, Hieracium amplexicaule, H. pulmonarioides, H. Jacquini, H. farinulentum, $H$. lanatum, Saxifraga aizoicles, S. Aizoon, S. rotundifolia, Campanula pusilla, Phyteuma orbiculare, Scrophularia Hoppii, Veronica urlicifolia, Linaria alpina, Erythronium Dens-Canis, Luzula nivea, Sesleria cærulea, Lasiagrostis Calamagrostis, Asplenium viricle, sans parler d'une multitude d'autres espèces qu'on voit assez communément dans les bois de Hètres de la région inférieure du Jura et du Bugey.

L'absence des susdites plantes dans notre Mont-d'Or peut s'expliquer de la manière suivante : l'observation directe et l'étude de leur distribution géographique montrent que le véritable centre de dispersion de toutes ces espèces se trouve dans la région des Sapins. On les voit, il est vrai, descendre au-dessous de cette zone; il en est même quelques-unes qui de temps en temps apparaissent sur les alluvions du Rhône en amont de Lyon. Il n'en est pas moins rrai que, puisque les susdites espèces appartiennent à la région des Sapins, elles ne peuvent se trouver dans notre Mont-d'Or, où elles n'auraient pu descendre que si cette région se trouvait située dans un massif montagneux comprenant des sommets de la zone des Sapins. Telle est l'explication plausible de la notable différerice qui existe à égale altitude, entre la Flore de nos montagnes juras:iques et celle du Jura, du Bugey et du Dauphiné. 
8II. Contraste entre la Floredu Mont-dor et les plateaux granitiques du Lyonnais

Il n'est aucun botaniste attentif qui n'ait été frappé de voir combien la végétation spontanéedes territoires granitiques de Mornant, Soucieu, Chaponost, Francheville, Tassin, Charbonnières, la Tourde-Salvagny, diffère de celle des terrains calcaires du Mont-d'Or lyonnais.

Cette différence est d'autant plus saisissante que ces derniers succèdent immédiatement, au-dessus de Limonest et de Chasselay, à la longue bande de terrains granitiques et gneissiques qu'on peut suivre depuis Chasselay et Limonest jusqu'au pied de la chaine de Riverie dans la vallée du Gier et qui, dans tout ce parcours, n'est interrompue que par l'îlot calcaire de Dardilly.

Nous avọs donné précédemment la liste des principales espèces du Mont-d'Or (voy. p. 139). Actuellement et sans entrer dans de trop longs détails, nous allons extraire, du tableau complet de la végétation des bas-plateaux lyonnais donné dans notre première partie, une énumération des plantes les plus communes sur ces territoires granitiques et qu'on ne trouve dans le Mont-d'Or que dans certaines localités, ainsi que nous le montrerons plus loin; ces espèces éminemment silicicoles sont :

Sarothamnus vulgaris.

Calluna vulgaris.

Gypsophila muralis.

Spergularia rubra.

Vicia lathyroides.

Digitalis purpurea.

Holcus mollis.

Hypericum pulchrum.

H. humifusum.

Orobus tuberosus.

Hypochæris radicata.

Veronica verna.

V. acinifolia.

V. præcox.

Anarrhinum bellidifolium.

Luzula maxima.
I uzula multiflora.

L. campestris.

Aira canescens.

A. flexuosa.

A. caryophyllea.

A. præcox.

A. divaricata.

Brassica Cheiranthus.

Teesdalia nudicaulis.

Vicia angustifolia.

Ornithopus perpusillus.

Scleranthus perennis.

Arnoseris minima.

Jasione inontaula.

Festuca tenuifolia.

Agrostis vulgaris. 
Vulpia pseudo-myuros.

V. myuros.

Nardurus Lachenalii.

Asplenium septentrionalc.

Dianthus Armeria.

Sedum maxinum.

Montia minor.

M. rivularis.

Corrigiola littoralis.
Peucedanum parisiense.

Bunium verticillatum.

Monchia erecta.

Ranunculus Philunotis.

Viscaria purpurea.

Crucianella angustifolia.

Ranunculus Chærophyllos.

R. hederaceus.

Silene Armeria.

Le contraste entre les deux végétations s'observe non seulement dans les points du pourtour du Mont-d'Or où les granites et gneiss sont en contact avec le lehm ou les éboulis des terrains jürassiques recouvrant les couches du trias, mais aussi entre les calcaires jurassiques et les grès triasiques qui, comme on le sait, constituent des sols siliceux.

Si, par exemple, des hauteurs de la Garenne, situees au-dessus de Poleymieux, on descend sur Chasselay, dès qu'on atteint les grès bigarrés, vers le bois de Pierre-Molière, on voit les Châtaigniers, les Pins, le Sarothamne, les Bruyères, l'Hierarium Pilosella, faire subitement apparition; le contraste est frappant'.

Mais dans l'intérieur même de la chaîne du Mont-d'Or, on peut observer des faits de contraste qui, quoique occupant une petite surface, n'en sont pas moins remarquables. Tous les géologues savent que, entre le mont Toux et le Verdun, s'étend sur le versant occidental du Narcel, une bande de grès bigarrés qui descend dans la partie supérieure du vallon de Poleymieux, où elle est recouverte par des bois de Pins, de Bouleaux et de Châtaigniers.

Indépendamment de ces trois arbres, on observe, sur cette bande de grès, une colonie d'espèces silicicoles exactement pareilles à celles qu'on peut relever, à peu de distance de là, sur les gneiss et les granites de Limonest et de Chasselay. Citons en particulier :

Hypericum pulchrum.

Orobus tuberosus.
Vicia angustifolia.

Genista germanica.

\footnotetext{
1 Ces gres remontent (rès haut sous le mont Verdun: et bien que recouverts par des éboulis de peu d'épaisseur, la végétation caractéristique les Pins, du Chàtaignier et d’autres espèces silicicoles suffit à dévoiler leur présence dans le sous-sol.
} 
Sarothamnus vulgaris.

Lonicera Periclymenum.

Saxifraga granulata.

Luzula maxima.

L. multiflora.

Carex pilulifera.

C. pallescens.

Aira flexuosa.

Festuca tenuifolia.

Anthoxanthum odoratum.

Potentilla Tormentilla.

Hieracium murorum.

Phyteuma spicatum.
Calluna vulgaris.

Veronica officinalis.

Teucrium Scorodonia.

Rumex Acetosella.

Maianthemum bifolium.

Agrostis vulgaris.

Melica unifiora.

Danthonia decumbens.

Pteris aquilina.

Polytrichum commune.

Dicranum scoparium.

Atrichum undulatum.

Divers Hypnum et ylocomium.

Nous ne devons pas omettre de signaler une complication qui se présente dans l'étude phytostatique du Mont-d'Or. On sait que dans les couches bajociennes de ce massif se trouve un calcaire renfermant de nombreux rognons de silex. Il arrive souvent que les habitants du pays amassent sur le bord des champs ces silex qu'ils appellent charveyrons; c'est précisement sur ces amas siliceux qu'on observe parfois l'existence de la Bruyère, du Genêt-à-Balai, du Danthonia decumbens et de quelques autres plantes qui, sans repousser complètement le carbonate de chaux, ne viennent que sur les sols à prédominance siliceuse.

On peut voir un exemple frappant de cette adaptation de la végétation au sol en descendant $d u$ mont Toux dans la dépression qui le sépare du mont Cindre, au sommet des vallons de Chatanay et de Saint-Romain : on aperçoit bientòt une bande assez étroite de Châtaigniers décrivant des sinuosités à la surface des cultures. Or, si l'on consulte la carte géologique du Mont-d'Or de MMI. Falsan et Locard, on reconnaît sans peine que ces sinuosites correspondent exactement à l'affleurement d'une couche riche en silice, le Calcaire à Bryozoaires.

Les mêmes observations s'appliquent aussi à la couche, située audessus du Calcaire jaune à entroques, qu'on appelle le Ciret. Sous ce nom, on désigne un calcaire fortement siliceux qui se délite à l'air et qui est impropre à la fabrication de la chaux. Si l'on explore les pelouses ou les bois qui les recouvrent, on ne devra pas s'étonner 
d'y trouver au milieu de plantes calcicoles, telles que Buxus sempervirens, Coronilla Emerus, Aster Amellus, Vincetoxicum officinale, etc., des espèces croissant de préférence dans les sols siliceux, comme Erica vulgaris, etc., des Chênes vigoureux, et même le Sarothamne, le Pteris aquilina; ce qu'on peut vérifier, par exemple, au--dessus des carrières de Couzon.

Du reste, les analyses de terres faites par Sauvanau montrent bien à quel point la terre végétale provenant de la désagrégation du Ciret est souvent pauvre en carbonate de chaux ${ }^{1}$ :

\begin{tabular}{llr} 
No 2. Résidu insoluble dans les acides. . . . . & 93,8 \\
Carbonate de chaux. . . . . . . . . & 0,4 \\
Autres matières solubles dans acides &. & 5,8 \\
\hline 100,00
\end{tabular}

Cet échantillon de terre a été pris sur le sommet du mont Cindre.

No 5. Résidu insoluble dans les acides. . . . . 97,2

Carbonate de chaux. . . . . . . . 0,4

Autres matières insolubles. . . . . 2,4

$\overline{100,00}$

Cet échantillon a étè pris à Poleymieux, au-dessus des Places.

Un autre échantillon de terre, pris comme le $\mathrm{n}^{\circ} 2$, sur le sommet du mont Cindre, présente une composition très différente des précédentes :

No 7. Résidu insoluble dans les acides. . . . 50,6

Carbonate de chaux. . . . . . . . 48,4

Autres matières solubles. . . . . . . 1

100,00

Ces résultats prouvent en outre que la composition chimique du sol est fort sujette à varier, même à de faibles distances, puisque nous voyons la terre $n^{\circ} 7$ contenir 48,4 p. $0 / 0$ de carbonate de

1 Ann. Soc. d'agric. de Lyon, t. VIII. 1ㅃㅎ. 
chaux, tandis que la terre $\mathrm{n}^{\circ} 2$, prise sur le même plateau du mont Cindre, n'en contient que $0,4 \mathrm{p} .0 / 0$.

Ces différences dans la composition chimique du sol, en rapport avec des variations de la Flore, expliquent l'erreur dans laquelle sont tombés les défenseurs de l'influence exclusivement physique du sol, comme Thurmann, lorsqu'ils donnent la présence d'espèces réputées silicicoles, dans une région calcaire, comme une preuve de l'inanité de l'influence chimique.

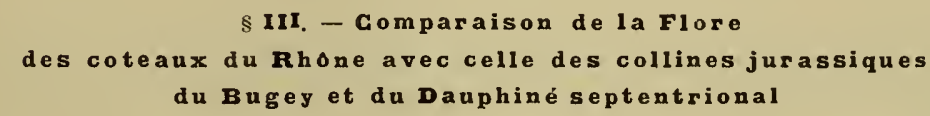

Si on examine comparativement la végétation des collines qui bordent le Rhône, en amont et en aval de Lyon, et celle des coteaux calcaires du Bugey et du pays de Crémieu et de Morestel, on ne tarde pas à remarquer qu'il existe une ressemblance assez notable entre les Flores de ces deux régions, pourtant si différentes l'une de l'autre par l'âge et par l'aspect extérieur des terrains.

On constate, en effet, sur les coteaux du Rhône, une grande prédominance d'espèces calcicoles, comme le montrent les séries suivantes :

$1^{\circ}$ Falaise méridionale de la Dombes : de Trévoux à Sathonay et Lyon, de la Pape à Meximieux.

Coronilla Emerus.

Buxus sempervirens.

Coronilla minima.

Ononis Columnæ.

Teucrium montanum.

Cerasus Mahaleb.

Geranium sanguineum.

Peucedanum Cervaria.

Psoroma fulgens.
Hutchinsia petræa.

Helianthemum salicifolium.

Dianthus Scheuchzeri.

Trifolium rubens.

Galium corrudæefolium.

Silene conica.

Fragaria collina.

Asperula galioides.

Aster Amellus.

$2^{\circ}$ Balmes-Viennoises ; Mollasse de Saint-Fons :

Dianthus Scheuchzeri.

Geranium sanguineum.

Hutchinsia petræa.
Helianthemum salicifolium.

Silene conica.

Ononis Columnæ. 
Fragaria collina.

Bupleurum aristatum.

Asperula galioides.
Psoroma fulgens.

Coronilla minima.

Veronica prostrata, etc.

$3^{\circ}$ Coteaux d'Écully, Vaise, Sainte-Foy, Beaunant, Beauregard, Irigny, Charly, etc.

Geranium sanguineum.

Ononis Columnæ

Peucedanum Cervaria.

Trifolium alpestre.

Fragaria collina.
Helianth. salıcifolium.

Silene Otites.

Asperula galioides.

Rubia peregrina.

Aster Amellus, etc.

Comme on le voit, les végétations de ceś coteaux ont la plus grande analogie entre elles; il est facile de vérifier aussi que ces espèces se retrouvent toutes sur les collines calcaires du Bas-Bugey et de Crémieu, comme :

Dianthus Scheuchzeri.

Linum angustifolium.

Geranium sanguincum.

Trifolium rubens.

T. alpestre.

Cerasus Mahaleb.

Rubia peregrina.

Globularia vulgaris.

Inula montana.

Aster Amellus.
Lithospermum purpureo-cæruleum.

Teucrium montanum.

Carex gynobasis.

Limodorum abortivum.

Orchis fusca.

Ophrys anthropophora.

$\mathrm{O}$ apifera, etc.

Epipactis lancifolia.

E. rubra.

Thesium divaricatum, etc.

Enfin, en comparant ces listes avec celles que nous avons données plus haut du Mont-d'Or, on peut constater sans peine une grande similitude, moins marquée cependant qu'avec le Bas-Buger.

Cette ressemblance est une preuve nouvelle de la prépondérance de l'influence chimique sur les actions physiques des roches.

Au surplus, on sait que les graviers qui forment la masse des terrains de transport des coteaux du Rhône se composent d'un mélange de cailloux quartzeux, dioritiques, granitiques et de fragments de roches calcaires venues des Alpes et des montagnes subalpines, jurassiques et néocomiennes de la Savoie et du Bugey. Or, comme les carbonates de chaux et de magnésie sont beaucoup plus solubles dans l'eau que les silicates, il en résulte que l'in- 
fluence chimique des roches calcaires l'emporte sur celle des roches siliceuses.

L'abondance du carbonate de chaux dans les eaux qui traversent les graviers des collines de nos environs est démontrée non seulement par l'analyse chimique, mais aussi par l'existence d'uı ciment calcaire qui, dans les conglomérats si communs autour de Lyon, a soudé les cailloux les uns aux autres.

Quant aux mollasses de Saint-Fons, leur richesse en chaux est bien démontrée par les analyses que nous en donnons plus loin.

On ne doit pas être surpris de voir que, à cause des rapports de voisinage, la végétation des coteaux du Rhône, en amont de Lyon surtout, offre une plus grande ressemblance avec celle des collines jurassiques de Crémieu et du Bugey, qu'avec la Flore du Montd'Or. En effet, on observe que plusieur's espèces plus ou moins communes sur les coteaux de la Pape, de Miribel, de Montluel, de Meximieux et des balmes de la rive gauche du Rhòne, sont rares ou nulles dans la chaîne du Mont-d'Or. Telles sont : Hutchinsia petræa, Helianthemun canum, H. pulverulentum, Cylisus capitalus, C. biflorus, Bupleurum aristatum, Scabiosa patens, Sc. suaveolens, Galium corrudxfolium, G. myrianthum, Linosyris vulgaris, Leontodon crispus, Scorzonera hirsuta, Micropus erectus, Ajuga genevensis, Carex nitidn, C. humilis, Koeleria valesiaca, etc.

Contrastes en petit. - Nous avons vu précédemment que dans la chaîne du Mont-d'Or, les éléments siliceux accumulés sur quel-ques points déterminent l'apparition d'une Flore silicicole. La même observation est, à plus forte raison, applicable aux terrains de transport des coteaux du Rhône. C'est ainsi que sur les sables du Mollard de Décines on remarque en grande abondance les espèces suivantes : Brassica Cheiranthus, Trifolium arvense, Corrigiola littoralis, Hypericum humifusum, Arnoseris pusilla, Hypochæris radicata, Jasione montana, Alkanna tinctoria, Myosotis hispida, Anarihinum bellidifolium, Rumex Aceto. sella, Corynephorus canescens, Vulpia pseudo-mynros, Psilurus nardoides, sans compter les Sarothammus vulgaris, Cal- 
luna et Pteris qu'on voit apparaître partout où prédominent les éléments siliceux. Les mêmes faits sont faciles à observer sur d'autres points des Balmes-Viennoises, à Montchat, à Bron, dans la plaine qui s'étend entre Génas et Heyrieux, et plus loin, en se dirigeant vers Saint-Quentin.

Tout le long du coteau de Lyon à Meximieux on peut constater aussi que, partout où le lehm devient siliceux, on voit apparaître le Sarothamne, les Andryala sinuata, Jasione montana, Anar.. rhinum bellidifolium, et disparaître les Globulaires et les autres plantes calcicoles.

Du reste l'analyse chimique vient corroborer les données de l'examen géologique.

Ainsi un échantillon de terre que nous avons prise à Bron, dans une localité où crô̂t le Genêt-à-Balai, contient sur 100 parties :

Carbonate de chaux. . . . . . . . . 3,26

Silice.. . . . . . . . . . . 69,20

Tandis que la mollasse de Saint-Fons, à Flore calcicole, contient sur 100 :

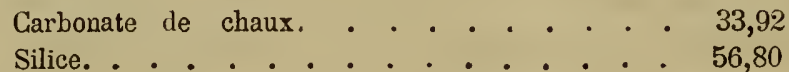

Quelques parties de cette mollasse, les points concrétionnés où croît le Dianthus Scheuchzeri, par exemple, contiennent même :

Carbonate de chaux. . . . . . . . . 46,42

Silice. . . . . . . . . . . . 48,32

La proportion de carbonate de chaux peut tomber dans quelques parties très sableuses, à 25 pour 100 et au-dessous; le sol est alor's envahi par des espèces psammophiles, comme Artemisia campes. tris, Plantago Cynops, Gypsophila saxifraga; mais les espèces silicicoles ne s'y rencontrent cependant jamais.

Nous avons vu plus haut que sur les coteaux du Rhône, le lehm revêtait quelquefois une Flore tout à fait silicicole. Lorsque son carbonate de chaux n’a pas été entraîné par la lixiviation due à 
l'infiltration des eaux de pluie, il supporte une Flore éminemment calcicole, sans Sarothamne, et à l'analyse on constate la présence d'une quantité considérable de calcaire, comme le montre l'exemple suivant, pris au-dessus de Beynost (Ain) :

Carbonate de chaux. . . . . . . . . 33,37

Silice. . . . . . . . . . . . . . 61,00

Sur les poudingues des mêmes localités. on observe un autre exemple de contraste en petit, fort intéressant: tandis qu'on voit le Lecidea geographica sur la face libre des cailloux roulés de quartzites alpins, le ciment calcaire qui les réunit est couvert de Barbula membranifolia, Psoroma fulgens, espèces éminemment calcicoles.

En résumé, la majeure partie des espèces qui vivent dans les environs de Lyon, sur les terrains de transport de la vallée du Rhône et de la Saône, sont calcicoles; ce n'est que par places isolées qu'on voit apparaitre les espèces silicicoles. Il en est tout autrement dans l'intérieur de la Dombes et de la Bresse à étangs. Là se montrent, dans les pâturages, les champs et les bois, toute la série des plantes que nous avons signalées précédemment sur les plateaux gneissiques et granitiques du Lyonnais. Cette similitude remarquable a une importance capitale dans la question qui nous occupe; car, sous le rapport des propriétés physiques du sol, il y a une diffẻrence considérable entre les argiles compactes, imperméables et éminemment perpéliques de la Dómbes et les sables perpsammiques qui résultent de la désagrégation des gneiss et des granites dans les plateaux du Lyonnais. La similitude de Flore dont il est question, et que nous développerons sous peu, ne peut donc être attribuée qu'à l'influence chimique. 


\section{(IHAPITRE II I}

AUTRES EXEMPLES DE CONTRASTES EN PETIT, PRIS EN DEHORS

DE LA REGION LYONNAISE

Les contrastes a de faibles distances, ont une si grande importance dans la question des rapports entre le sol et la végétation que, au risque d'encourir le reproche de trop élargir mon sujet, je ne puis résister au désir de corroborer les faits que nous offre l'examen de la contrée lyonnaise par des exemples semblables empruntẻs à d'autres régions.

M. Planchon ${ }^{1}$ a signalé le contraste singulier de la végètation des dolomies du Gard et de l'Hérault avec celle des schistes talqueux environnants. Tandis que sur ceux-ci on constate la présence de la cohorte bien connue des plantes silicicoles des Cévennes, au contraire, sur les dolomies apparaissent plusieurs espèces calcicoles, parmi lesquelles il faut citer en particulier :

* Ethionema saxatile.

* Lieruera saxatilis.

* Daphne alpina.

* Potentilla caulescens.

* Hicracium amplexicaule.

Lavandula vera.

Chrysanthemum graminifolium.

Sedum anopetalım.

Campanula speciosa.
* Hieracium saxatile.

* Erinus alpinus.

* Athamanta cretensis.

* Rhamnus alpina.

* Draba aizoides.

Alenaria hispicla.

Arenaria tetraquetra.

Il)eris saxatilis.

Parmi les espèces ci-dessus mentionnées, celles que nous avons marquées d'un astérisque se trourent ausi sur les calcaires jurassiques et néocomiens du Jura, du Bugey, de la Savoie et du Dauphiné.

L'Iberis saxalilis existe sur les calcaires jurassiques des Cor-

1 Sur la régetation spéciale des dolomies du Gard et de l'Hèrault. Bull. Soc. bot. de Fr, t. I, 185 ́. 
bières et de l'Aveyron; on le trouve sur les calcaires jurassiques et néocomiens de la Provence.

L'abondance du Lavandula vera et du Sedum anopelalum dans toutes les régions calcaires du Midi de la France est bien connue des botanistes.

Le Campanula speciosa est aussi une espèce calcicole de toute la chaîne des Pyrénées orientales et centrales, ainsi que des Corbières; elle remonte mềme jusque dans la région jurassique de Mende (Lozère).

L'A renaria tetraquetra se montre sur les calcaires jurassiques et néocomiens de la Provence et des Alpes-Maritimes.

Enfin, le Chysanthemum graminifolium s'observe sur les mêmes terrains dans la Provence, les Alpes-Maritimes, les Corbières; on le voit aussi en très grande abondance sur les calcaires jurassiques de la Charente-Inférieure.

M. Boulay ${ }^{1}$, suivant les traces de M. Planchon, a constaté des faits semblables dans les Vosges. La Flore des grès vosgien et rouge des environs de Saint-Dié est complètement silicicole, comme on peut le remarquer en lisant l'énumération des plantes citées par M. Boulay. Cependant sur quelques points on voit apparaître un ensemble de plantes calcicoles qui contrastent avec la végétation environnante; et si on examine le sol, on voit que les espèces calcicoles ne se montrent que sur des îlots de dolomies enclavées dans le grès.

Déjà Kirschleger ${ }^{2}$ avait signalé la présence de quelques espèces jurassiques sur les dolomies de Sainte-Marie aux Mines, de Soulzmatt et de Wintzfelden, sur le versant alsacien des Vosges; Facchini ${ }^{3}$ avait constaté aussi que, dans les Alpes de Fassa et de Fiemme, où les dolomies alternent avec le porphyre augitique, on ne peut s'empêcher d'être frappé de la différence de végétation ‘qui existe sur les deux terrains.

M. Godron a fait connaitre le contraste qu'on observe dans la

1 Notice sur la Géogr. bot. des environs de Saint-Dié. Besançon, $1 \$ 66$.

: Flore d'Alsace, t. III, I. 19.

3 Nuovi Annali delle scienze natural: . II, 1838. 
vallée de Vernet-les-Bains (Pyrénées-Orientales), entre la Flore les terrains granitiques et gneissiques, et celle du Bois-Pinat, petite enclave calcaire entourée de toute part de roches feldspathiques. Sur cet îlot, on peut voir plusieurs des espèces calcicoles qui se retrouvent sur les collines calcaires de la Trencada d'Ambulla, dans la vallée de l'Agly.

Boreau dit qu'à l'extrémité d'un faubourg d'Angers s'élève un monticule de calcaire dévonien enclavé dans les schistes, sur lequel les botanistes d'Angers avaient coutume d'aller chercher certaines espèces calcicoles qui manquent dans le reste de la contrée.

A travers le Bocage vendéen, composé en majeure partie de granite et de schistes argileux anciens, apparaissent çà et là quelques îluts calcaires dont la végétation diffère complètement de celle des terrains siliceux environnants, ainsi que l'ont très bien remarqué Letourneux et Lloyd ${ }^{1}$. Les mêmes faits se reproduisent dans les petits bassins calcaires de Chantonnay, de Puyrinsens, de la GrandeRhé, près le Vouvant, clans la Plaine, dans le Marais, ainsi que dans les petits bassins calcaires de Machecoul et d'Arthon.

La forêt de Fontainebleau est une localité classique pour les géologues et pour les botanistes. On sait que le sol de cette forêt est, en majeure partie, constitué par des grès et des sables appartenant à l'étage appelé sables moyens de la formation parisienne.

Les botanistes, de leur côté, ont depuis longtemps remarqué que la végétation spontanée de la forêt de Fontainebleau offre une grande prédominance d'espèces silicicoles. La Flore bryologique et lichénologique des rochers est particulièrement instructive à cet égard.

Pourtant, sur quelques points, particulièrement au mail de Henri IV, au carrefour des Hêtres, et au mont Perreux, apparaissent quelques plantes réputées à bon droit calcicoles, dont on trouvera l'énumération dans un Mèmoire de M. Fliche 2.

Or il résulte des observations faites par divers naturalistes que

1 Bull. Soc. bot. de Fr., t. VIII, 1861, p. 92. - Flore de l'Ouest. Nantes, 1868.

2 Du sol des envir. de Fontaingbleau et de ses relations avec la vegétation. Mém. Soc. des sc. Nancy, 1870. Voyez aussi: Rapport sur l'herbor. dans la forêt de Fontai. nubleau, par de Schönefeld. Buıll. Soc. bot. F). t. II, 185 J. 
partout où apparaissent les espèces calcicoles, on trouve, soit à la surface, soit à une faible profondeur, des couches de calcaire lacustre.

On comprend d'ailleurs que le mélange des éléments calcaires avec les sables siliceux n'a pas toujours lieu dans les mêmes proportions, et il est fort curieux de constater que le nombre des espèces calcicoles est toujours en relation directe avec la quantité de carbonate de chaux contenue dans la terre.

Mais voici un fait bien plus remarquable encore. Dubourg d'Isigny ${ }^{l}$ dit que les coteaux crayeux de la Seine présentent une grande diffërence dans leur Flore suivant que le calcaire affleure à la surface du sol ou qu'il est reconvert de sables de transport. Comme exemple de ces variations, Dubourg ajoute: « J'ai pu cueillir d'une main la Digitale jaune ì petites fleurs, et de l'autre la Digitale pourprée? ${ }^{2}$ )

J'arrive à un fait de contraste qui mérite d'être rappelé à cause des conséquences qu'en a tirées Thurmann pour combattre la doctrine de l'influence chimique du sol.

L'Alp ou (Alb) du Wurtemberg est composé de couches cal.. caires appartenant aux divers étages de la formation jurassique. La Flore de l'Alp est calcicole, ainsi que l'ont très bien observé tous les botanistes wurtembergeois, et en particulier Martens et Kemmler ${ }^{3}$. Sous ce rapport elle forme un contraste frappant avec la Flore du Schwarzwald, lequel est constitué en grande partie par des couches de gneiss et des masses granitiques.

Cependant sur quelques points de l'Alp, on voit apparaitre quelques-unes des espèces silicicoles du Schwarzwald, entre autres, Betula alba, Sarothamnus vulgaris, Calluna vulgaris, Luzula albida, Arnica montana et plus rarement Digitalis purpurea.

1 Aperçus sur la Géologie et la Flore de l'arr. de Vire. Mém. Soc. linn. Normandie, t. VI, 1838.

2 Nous rappelons qu'on peut constater des exemples de semblables contrastes dans maints endroits des régions calcaires du Jura, du Buger, etc.; les roches jurassiques et crétacées y sont souvent recouvertes par des dépôts erratiques, siliceux, sur les. quels on voit apparaître le Chảtaigner et d'autres silicicoles.

3 Flora von Württemberg und Hohenzollern. Tübingen, 1S65, 3• édit. 
D’après Thurmann ${ }^{1}$, ces espèces qu'on ne voit habituellement que sur les sols siliceux, se montrent à l'Alp, seulement sur certaines dolomies, lesquelles par leur désagrégation produisent un sable qui se rapproche, au point de vue des propriétés physiques, du sol psammique formé de la décomposition des grès, des gneiss et des granites. Preuve évidente, ajoute Thurmann, que le terrain agit sur la dispersion des plantes par les seules influences mécaniques et physiques, et nullement par l'effet de sa composition chimique.

L'objection ainsi formulée parThurmann était restée sans réponse. M. Contejean, qui avait réfuté les autres arguments de son maître, s'ètait borné à dire que “ les renseignements donnéspar Thurmann sur les localités de l'Alb du Wurtemberg ne sont pas assez précis pour qu'il soit permis de tirer des conclusions de faits insuffisamment décrits. Du reste, ajoutait M. Contejean, “ ne connaissant pas l'Alb wurtembergeois, je ne tiendrai aucun compte de cet exemple. "

Cependant il y avait une chose bien simple à faire, c'ètait d'examiner qu'elle est la nature chimique des dolomies qui avaient servi à Thurmann pour bâtir l'édifice de son argumentation restée sans réplique. Il n'était même pas besoin d'aller étudier sur place les susdites dolomies, car le Jura wurtembergeois est une des régions les mieux explorées. Il suffisait de chercher ce qu'en ont dit les géologues wurtembergeois; c'est ce qu'a fait M. Saint-Lager.

Or il est facile de voir, en lisant les ouvrages de Fried. d'Alberti ${ }^{2}$, de Fréd. de Mendelsloh ${ }^{3}$, de Hehl ${ }^{4}$, de Quenstedt ${ }^{5}$ et d'A $\mathbf{a -}$ chenbach $^{6}$, qu'il existe, on plusieurs endroits de l'Alb, des amas formés: $1^{\circ}$ d'une terre argileuse d'un jaune rougeâtre, $z^{\circ}$ de sables blancs, accompagnés de silex jaspiformes, de boules calcédonieuses et de minerais de fer.

1 Essai de phytostatique, t. I, p. 234.

2 Die Gebirge d. k. Württemberg. Stuttgart, 1826, p. 300. Halurgische Geologie. Stuttg., 1852, p. 205, 241

3 Mémoire sur la constitut. géol, de l'Alb du Wür'temberg, Mém. Soc. hist.nat., Strasbourg, 1835. t. II, p. 8.

4 Die geognostische Verhältnisse Württembergs. Stuttg., 1850, p. 106, 143.

s Das Flözgebirge Württembergs. Tübing., 1843.

- In Württemb. Jahreshefte, t. XV, 1859, p. 103. 
Cette formation (Bohnerz des géologues allemands, dépôts sidérolithiques des géologues français) occupe tantôt des excavations en entonnoir, creusées dans le calcaire jurassique, tantôt des crevasses. Parfois les dépôts sableux s'étendent en couches sur le plateau de l'Alb.

Les calcaires et dolomies qui environnent ces dépôts ont été silicifiés au point de faire feu lorsqu'ils sont frappés par le pic; ils se désagrégent en un sable plus ou moins fin.

C'est précisèment sur ces dolomies silicifiées et sur les dépôts sableux et sidérolitiques, et nulle part ailleurs, qu'on observe dans l'Alb du Wurtemberg, la Bruyère, le Genêt-à-Balai, la Luzule blanche, l'Arnica, le Bouleau et la Digitale.

La prétendue dolomie 'dont Thurmann avait méconnu la vẻritable composition chimique, renferme, suivant les échantillons, de 27 à 34 et même jusqu'à 96 pour cent de silice. La terre qui résulte de sa désagrégation est encore plus pauvre en carbonates de chaux et de magnésie ; elle ne contient que 0,7 à 1,3 pour cent de carbonate de chaux; ce qui s'explique aisémentsi l'on considère que le carbonate de magnésie est assez soluble dans l'eau chargée d'acide carbonique et que la lixiviation de ce sable a été favorisée par son extrême division.

Du reste, les dépôts sidérolithiques ne sont pas particuliers au Jura wurtembergeois ; il en existe sur plusieurs points du Jura helvétique et français, ainsi que dans les montagnes calcaires de l'arrondissementd'Annecy (Savoie). M. Saint-Lager, ayant visité quelques-unes des localités où existent les susdits dépôts, a constate que partout ils supportent une Flore silicicole complètement contrastante avec celles des calcaires environnants.

FIN 



\section{EXPLICATION DES GARTES}


CARTE D'ENSEMBLE DE LA RÉGION LYONNAISE

A. ZoNe SIliceuse :

Sarothamnus vulgaris, Racomitrium canescens, Lecidea geographica.

1. Vallées et Bas-Plateaux du Lyonnais.

2o Région d'étangs du plateau de la Dombes.

B. Zone calcaire :

Buxus sempervirens, Grimmia crinita

Placodium callopismum.

$3^{\circ}$ Mont-d ${ }^{\circ} r^{\cdot}$ lyonnais ;

$4^{\circ}$ Coteaux du Rhône et de la Saône;

a. Côtière occidentale et méridionale de la Dombes ;

b. Balmes-Viennoises;

c. Coteanx d'Oullins à Givors.

G. ZONE NIXTE :

Bas-Dauphiné ; - Plaine de la Valbonne, ete. 


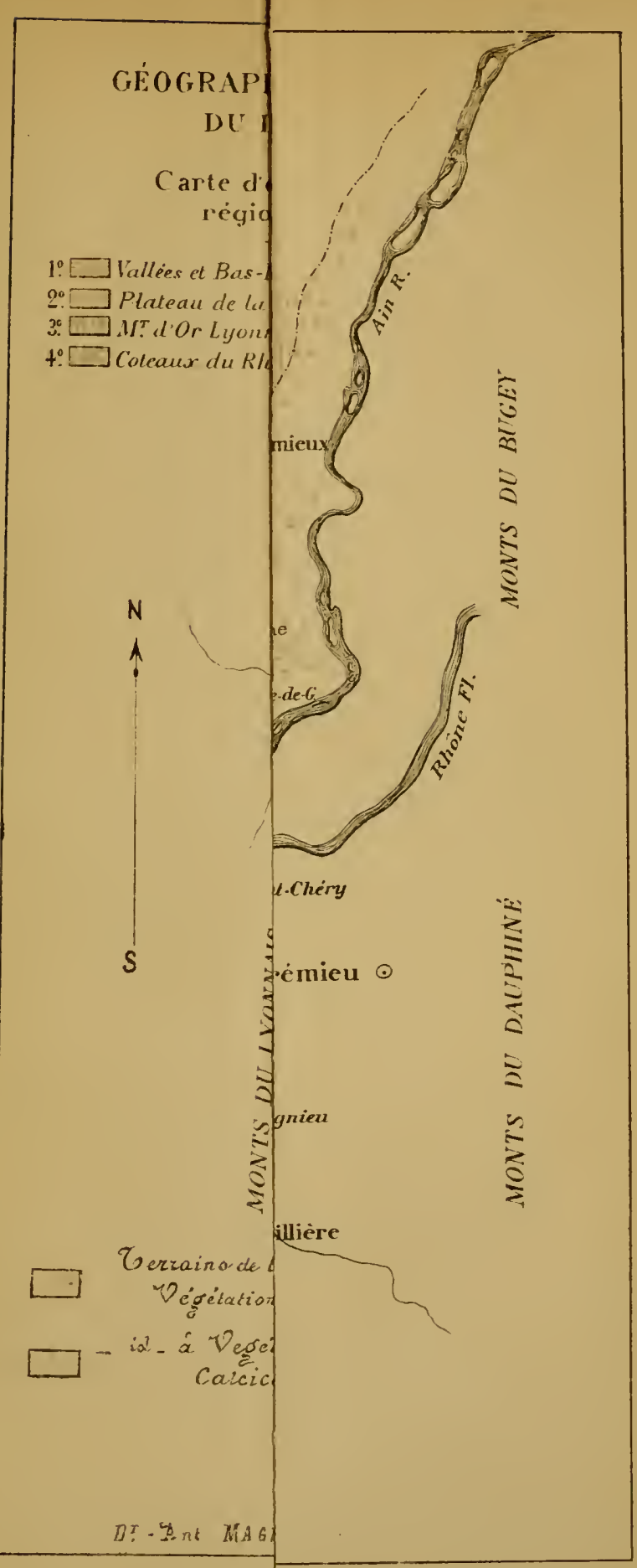




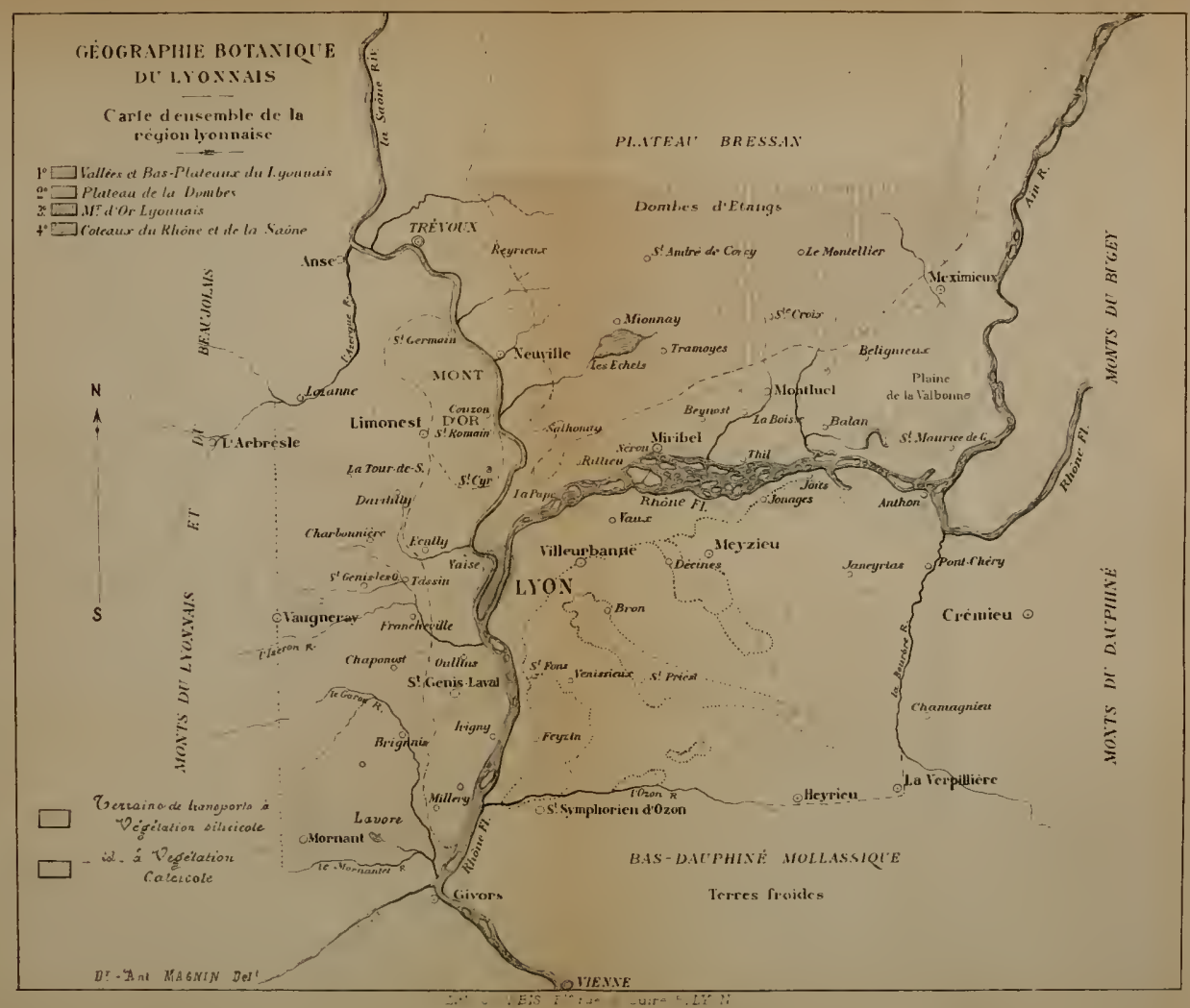



-. - Limite occidentale des terrains de transport, d'origine alpine, daus Ia région lyomnaise (alluvions glaciaires, bone glaciaire et lehm); elle divise chacun des bas-plateaux en deux parties:

10 Partie occidentale, à sol granitique ou gneissique, à Flore éminemment silicicole;

20 Partie orientale, à sol variable, granitique ou gneissique sur les bords, formé, dans les parties supérieures, par des cailloux roulés, généralement de quartzites alpins, dont la végétation est aussi silicicole.

En se rapprochant des vallées de Tassin, de Francheville et de Beaunant, les éléments calcaires deviennent, daus quelques localités, prédominants et des jlantes calcicoles apparaissent. 



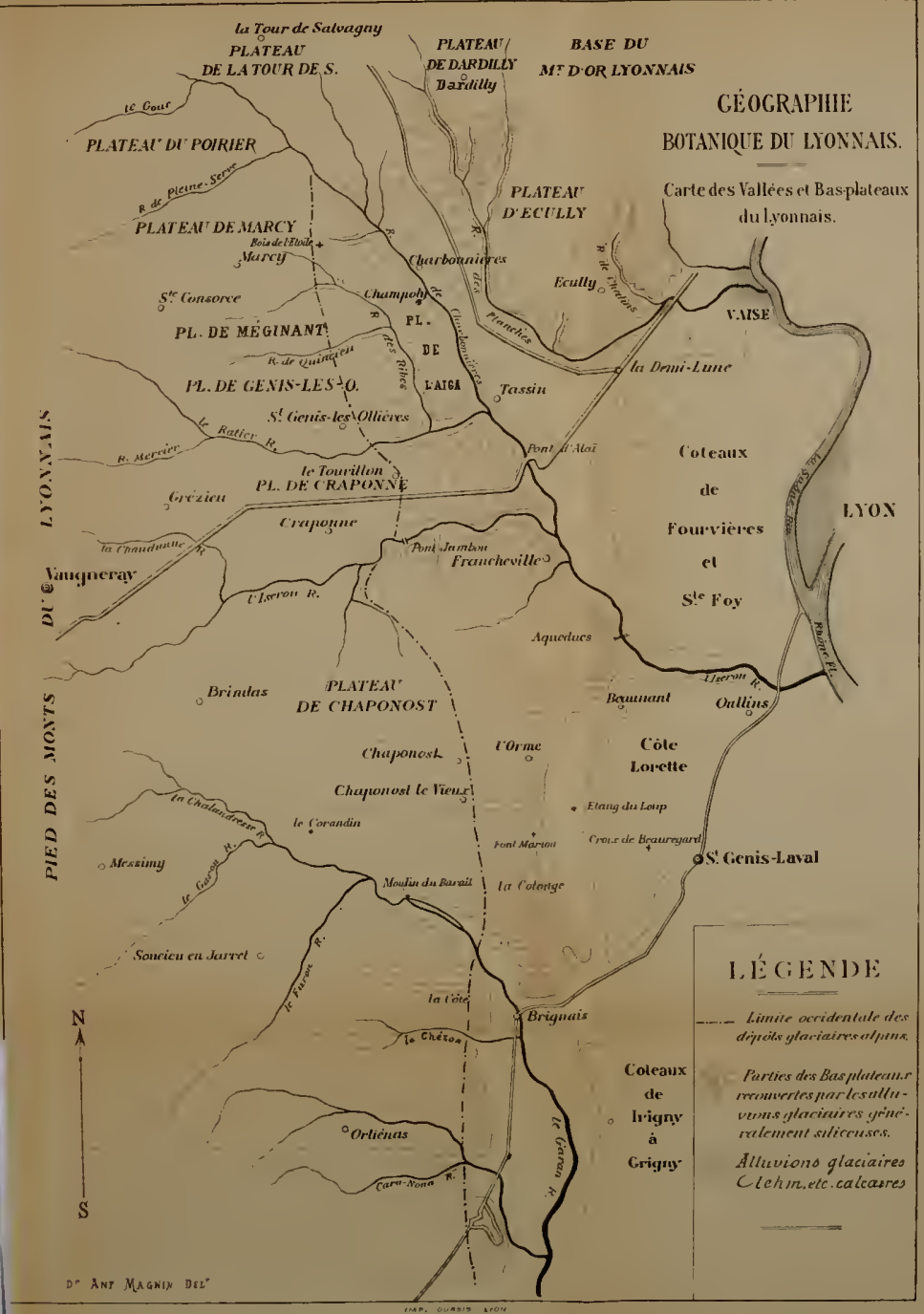



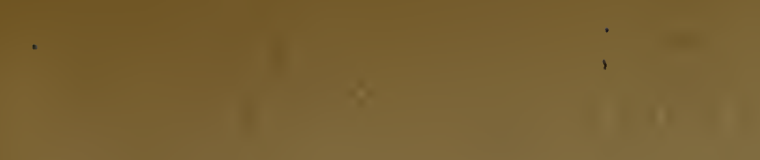

aly.

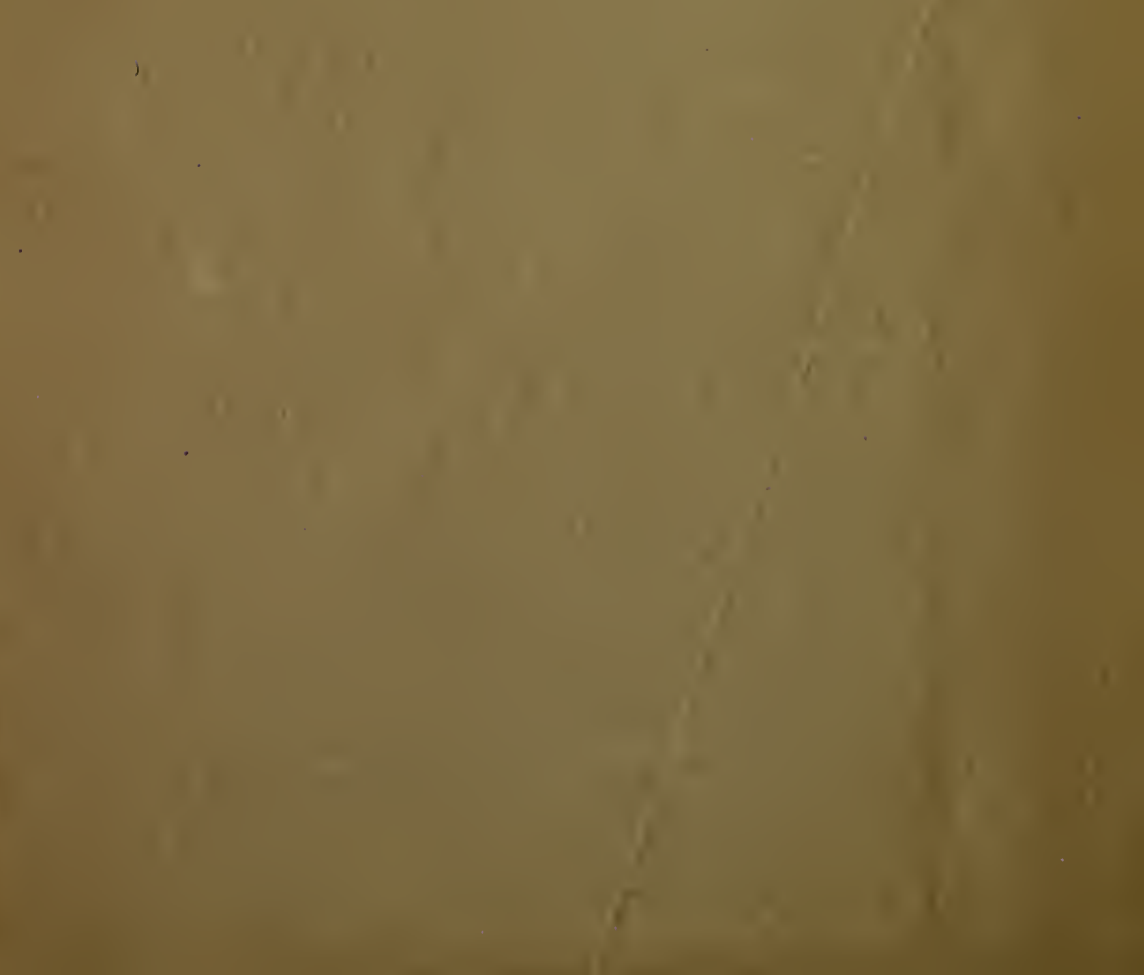




\section{LIBRAIRIE I.-B. BAILLLEE \& FIL,}

ARDOINO. - Flore analytique du département des Alpes-Maritimes, 1E79. in-\&.

$8 \mathrm{fr}$.

BRAS. - Catalogue des plantes vasculaires du département de l'Aveyron, 1877. gr. in-8.

$8 \mathrm{fi}$.

CAUVET. - Cours élémentaire de bota. nique. 1879.1 vol. in-18 jesus, iv0 pages avec 617 figures.

$7 \mathrm{rr}$.

CONTEJEAN. - Éléments de géologie et et de paléontologie. 1874 , in-8 le $75 \%$ pages, avec 467 fig., cart.

$16 \mathrm{fr}$.

DUCHARTRE.- Élements de botanique, comprenant l'anatomie, Iorganographic, la physiologie des plantes, les familles narelles et la géographie bolanique. $2^{\circ}$ édition. $18 \%$, 1 vol. in-s de vur-1272 pages, avec 541 fig.

$20 \mathrm{fr}$,

GikRIIAIN (de saint-Pierre). - Dictiollnaire de botanique, comprenant la description des fannlles naturelles les pro priètés médicales et les usages économ:ques des plantes etc., 1870, in-8. 25 fr.

GRENIER. - Flore de la chaîne jurassique, par Car. Grisier. Eilition comflète, précedee de la Recue de la Floric lles monts Jura. Paris, 1865-75. 3 parties formant 1 vol. itl-S de $10 ! 12$ pages. Car. tonne.

$12 \mathrm{fr}$.

GRISEBAGH (A.). - La végètation dil globe, d'apres sa disposition suivant les chmats; esquisse l'une géographie comparée des plantes, par A. Grisebacin, traduit par P. DE Tcinн.iтcheF, et accompayni d'une carte générale des domaines de vèutation. 187i-78, 2 rol. in-S de 700 pages.

$30 \mathrm{fr}$.

KIRSCHLEGER, - Flore vogés0-rhénane. ou Description les plantes qui croissen naturellement dans les Yosges et clans la vallèe du Rhin. 1870, 2 rol. in-18 jèsus.

$15 \mathrm{fr}$.

L.MMOTTE (M.nTraL). - Catalogue des plantes vasculaires de l'Europe centrale, comprenant la France, la Suisse, l'Allemagne. 18.1\%, in.S le 104 lages. 2 fr. 50
LECOQ (Clt.). - Études sur la Géographie botanique de l'Europe, el en particu'। r sur la régétation du plateau central de in France. 1\$54-5S, 9 vol. crr. in-s, ar: 3 planches coloriées.

(5)

MOQUIN-TANDON. - Élements de botanique médicale, contensm l la desciiptio1 de's végetaux utiles à la médecine ê des espèces nuisibles à thomme, rénéneuses ou parasiles. $3^{\mathrm{c}}$ édition. Paris, $1876.1 \mathrm{~m}$. in-18 jèsus arec $1: 33$ lig.

(i) 1 .

RLNAUL'1. - Contributions à la paléontologie végétale. Études sur le siryillu spinulosa et sur le genre Myeinteri. par B. Rexaclt, docteur es sciences nitturelles. Paris, $18 \% 6$, in-4 de 52 pages, are 12 planches noires et colorièes. $10 \mathrm{fr}$.

SCHIMPER. - Traité de paléontologie végétale, ou la flore du nionde primitil tans ses rapports avec les formations geologiques et la tlore du mon to ar tuel. 1 is i.. $187-4,3$ vol. gr. in- $\&$, arec atlas d 110 planrhes in-rôlio.

15) $1 \mathrm{r}$.

VERLOT. (B.) - Guide du botaniste herborisant. Cunseil sur la recolte des plantes. la prepraration des herbiers, lexploration des plantes pharénogames et cryptogam . et les herborisations aux environs de P. ris, dans les Ardennes, la Bourgogne, la Provence, le Languedoc, les Py rénées, les Alpes, l'Auvergne, les Vosges, an bor 1 tr la Manche, de l'Océan et de la mer Méli. terranee, par Bern.rod VirLot, chef it l'École de botanique au MIuséum d'hist nim ıaturelle de Paris. 2 é litıon. 187!1. 1n-I9, 650 paces aver figures. cartomne. $6 \mathrm{ir}$.

\section{VERLOT (J.-B.). - Catalogue raisonie} des plantes vasculaires du Dauphiné par M. J.-B. TERLot, jardinier en chef, directeur du Jardin des piantes de Gren. ble. Grenoble, 1872, 1 vol. in-S de vir bus lages.

$12 i$

WATELET (Ad.). - Description des plantes fossiles du bassin de Paris. Par 1E66, 1 vol. in-1, $24: 4$ pages, avec atlas d to planclies Ourragre complet publiẻ en i livraisons, cartonné.

$6011^{\circ}$. 



\section{QK313.M251}

QK313 M251
Magnin, Antoine/Recherches sur la geogra

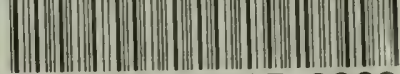

35185001070968 
